\title{
The Einstein database of IPC x-ray observations of optically selected and radio-selected quasars, 1.
}

\section{Citation}

Wilkes, Belinda J., Harvey Tananbaum, D. M. Worrall, Yoram Avni, M. S. Oey, and Joan Flanagan. 1994. "The Einstein Database of IPC x-Ray Observations of Optically Selected and Radio-Selected Quasars, 1." The Astrophysical Journal Supplement Series 92 (May): 53. doi:10.1086/191959.

\section{Published Version}

doi:10.1086/191959

\section{Permanent link}

http://nrs.harvard.edu/urn-3:HUL.InstRepos:30212177

\section{Terms of Use}

This article was downloaded from Harvard University's DASH repository, and is made available under the terms and conditions applicable to Other Posted Material, as set forth at http:// nrs.harvard.edu/urn-3:HUL.InstRepos:dash.current.terms-of-use\#LAA

\section{Share Your Story}

The Harvard community has made this article openly available.

Please share how this access benefits you. Submit a story.

\section{Accessibility}


THE ASTROPHYSICAL JouRNAL SUPPLEMENT SERIES, 92:53-109, 1994 May

(c) 1994. The American Astronomical Society. All rights reserved. Printed in U.S.A.

\title{
THE EINSTEIN DATABASE OF IPC X-RAY OBSERVATIONS OF OPTICALLY SELECTED AND RADIO-SELECTED QUASARS. I.
}

\author{
Belinda J. Wilkes, ${ }^{1}$ Harvey Tananbaum, ${ }^{2}$ D. M. Worrall,${ }^{3}$ Yoram Avni, ${ }^{4}$ M. S. Oey, ${ }^{5}$ And Joan Flanagan ${ }^{6}$ \\ Received 1993 April 19; accepted 1993 August 26
}

\begin{abstract}
We present the first volume of the Einstein quasar database. The database includes estimates of the X-ray count rates, fluxes, and luminosities for 514 quasars and Seyfert 1 galaxies observed with the Imaging Proportional Counter (IPC) aboard the Einstein Observatory. All were previously known optically selected or radio-selected objects, and most were the targets of the X-ray observations. The X-ray properties of the AGNs have been derived by reanalyzing the IPC data in a systematic manner to provide a uniform database for general use by the astronomical community. We use the database to extend earlier quasar luminosity studies which were made using only a subset of the currently available data. The database can be accessed on internet via the SAO Einstein on-line system ("Einline") and is available in ASCII format on magnetic tape and DOS diskette.
\end{abstract}

Subject headings: galaxies: Seyfert — quasars: general — X-rays: galaxies

\section{INTRODUCTION}

Einstein observations have shown that most, if not all, quasars are luminous X-ray sources ( Tananbaum et al. 1979; Avni \& Tananbaum 1986, hereafter AT86). The Einstein data have been used to determine the X-ray properties of individual quasars as well as to study the characteristics of statistically welldefined or complete samples. A substantial body of literature (references in text below) presents data on quasars selected for $\mathrm{X}$-ray observation on the basis of a wide range of characteristics (e.g., optical flux, optical luminosity, redshift, radio characteristics, etc.), providing convincing evidence for the correlation of X-ray emission with optical emission and for the presence of excess X-ray luminosity in radio-loud quasars. In addition, Einstein observations of statistically well-defined samples ( see $\S 3$ ) have been used to carry out analyses of quasar luminosity and evolution functions in the X-ray band.

Most of these Einstein observations of quasars (and all of those reported in this paper) were carried out with the Imaging Proportional Counter (Giacconi et al. 1979). The Imaging Proportional Counter (IPC) was well suited for this observational program by virtue of its high throughput, its better than 30 " positional accuracy for point sources, and its modest energy resolution which enabled us to determine fluxes and luminosities over a reasonably well-defined energy band.

A large number $(\sim 1000)$ of quasars and active galaxies (AGNs) were observed from 1978 to 1981 as part of many different observing programs with the IPC. Many of these data have been analyzed and published elsewhere under the original

\footnotetext{
${ }^{1}$ Harvard/Smithsonian Center for Astrophysics, 60 Garden Street, Cambridge, MA 02138. Electronic mail: wilkes@cfa.harvard.edu.

${ }^{2}$ Harvard/Smithsonian Center for Astrophysics, 60 Garden Street, Cambridge, MA 02138. Electronic mail: ht@cfa.harvard.edu.

${ }^{3}$ Harvard/Smithsonian Center for Astrophysics, 60 Garden Street, Cambridge, MA 02138. Electronic mail: dmw@cfa.harvard.edu.

${ }^{4}$ Harvard/Smithsonian Center for Astrophysics. Deceased.

${ }^{5}$ Steward Observatory, Tucson, AZ 85721.

Electronic mail: oey@as.arizona.edu.

${ }^{6}$ Harvard/Smithsonian Center for Astrophysics, 60 Garden Street, Cambridge, MA 02138.
}

scientific programs involved. Due to differing analysis procedures and scientific aims, the results are of varying quality and scattered throughout the literature.

In this paper we present the first part of the Einstein quasar database. The database includes estimates of or upper limits to the X-ray count rates and errors, fluxes, and luminosities for 514 previously known optically selected or radio-selected quasars and Seyfert 1 galaxies for which targeted observations were made with the IPC. The results have been derived by reanalyzing, in a systematic manner, the current ( Rev 1B) version of the IPC-processed data to provide a uniform database for general use by the astronomical community. The data are presented in a series of tables as follows: Table 1 gives the basic information on each quasar; Table 2 gives details of the Einstein observations; Tables $3 \mathrm{~A}-3 \mathrm{C}$ give the $\mathrm{X}$-ray fluxes and luminosities for five different assumed energy indices: $0.0,0.5$, 1.0, 1.5, and 2.0; Tables 5A and 5B list objects and observations missing from our database; Table 6 gives optical magnitudes and luminosities.

\section{THE SAMPLE}

The IPC quasar observations have been divided into two subsets since the details of the analysis procedure are different in the two cases. The first subset is presented here and includes primarily quasars and Seyfert 1 galaxies which were targets of IPC observations. The objects in this "target sample" mostly appear on-axis in the images. The target sample also includes several fields with multiple known quasars where the entire group is the selected target and most appear off-axis in the image. The second subset includes radio-selected and optically selected quasars which were observed because they lie in the field of view of another IPC target. These latter objects, which we estimate as roughly equal in number, will be reported in a later paper. Some nontarget sources, which have already been analysed and are included in this paper, are noted in Table 2.

The current sample contains 636 observations of $514 \mathrm{ob}-$ jects. We include all sources which are classified as quasars or Seyfert 1 galaxies in the compilations of Hewitt \& Burbidge 
(1987, 1989) and Véron-Cetty \& Véron (1987). We also include objects classified as quasars in the Einstein Observatory Catalog of Observations ( the "Yellow Book," 5th edition; Section III, Tables 4.1 and 4.3 ) with the exception of objects obviously misclassified (BL Lac, objects, galaxies, etc.), without a redshift in the available literature, or which were missed due to satellite pointing problems or incorrect positions. Such objects are all noted in Table 5A (see below for additional details).

For X-ray-bright objects observed on multiple occasions to study possible time variability, we normally use the initial ("survey") flux observation in order to avoid introducing biases into our analysis. For objects observed more than once in a "survey" or serendipitous mode, we normally select the longest exposure for further analysis. In a few instances the selection of a "primary" observation is made on an arbitrary basis. In any case, whenever there are multiple observations, the one selected for further analysis is indicated by a " 1 " in the Note columns of Tables 2 and 6.

Basic information is given in Table 1 with the quasars in order of increasing source right ascension and including various common names, optical celestial coordinates (1950), references, redshift, notes, and sample membership. The coordinate designation given in the first column follows IAU convention, is unique to each object, and is used throughout the paper to identify that object in the database. Letters have been added when necessary to ensure uniqueness internal to this database, but no attempt was made to conform to the efforts of other authors to do the same.

As a whole our sample is incomplete, although it contains a few complete subsamples: 64 PG quasars (Tananbaum et al. 1986); 33 3CR radio quasars (Tananbaum et al. 1983); and 30 Braccesi BF quasars (Marshall et al. 1984). These complete subsamples are indicated in Table 1. The PG quasars are an unbiased subset selected from the original Bright Quasar Survey (Schmidt \& Green 1983); the current sample contains two fewer PG X-ray observations than reported by Tananbaum et al. (1986) due to the exclusion of one HRI observation and one IPC measurement confused by nearby X-ray sources (see Table 5A). The 3CR and BF subsets are both complete, fluxlimited samples (Schmidt 1968; Tananbaum et al. 1983; Braccesi, Lynds, \& Sandage 1968; Marshall et al. 1984). The remainder of our sample is a heterogeneous mixture of quasars and Seyfert 1 galaxies which were observed by many different investigators for various scientific reasons.

\section{THE DATABASE}

\subsection{Source Detection and Flux Estimates}

Throughout this paper, we use the standard IPC PI (pulseheight invariant), broad-band $(0.16-3.5 \mathrm{keV})$ images and background maps from the data processed with the current, Rev 1B, version of the standard processing software (described in Harnden et al. 1984). Fluxes were determined using definitive versions of the effective area table and the gain calibration (Harnden et al. 1984). The data were analyzed using a fully automated procedure to ensure uniformity and consistency. A list of all the observations, including Einstein sequence number ( unique identifier for each Einstein observation), observation dates, count rate and uncertainty, livetime, angle off-axis, and detector gain, are given in Table 2 in increasing order of
RA. The coordinate designation and IPC sequence number are used in subsequent tables to identify uniquely each object/observation pair.

Quasar optical positions are taken in order of preference from Schmidt \& Green (1983) for PG objects, from Hewitt \& Burbidge $(1987,1989)$, and from Véron-Cetty \& Véron (1987) (Table 1). X-ray locations are accurate to $\pm 20^{\prime \prime}(1 \sigma)$ as determined by comparing the X-ray and optical positions for the X-ray-detected quasars in the PG sample. This is consistent with the detailed study of position accuracy reported in the IPC Specifications (Harnden et al. 1984). The X-ray analysis was performed using the optical position for X-ray nondetections and the X-ray centroid for X-ray detections within $1^{\prime}$ $(3 \sigma)$ of the optical position. The use of the X-ray centroid allows an accurate estimate of the X-ray flux of the source.

The presence/absence of $\mathrm{X}$-ray emission was determined using a standard $2.4 \times 2$. 4 detect cell on the IPC broad-band $(0.16-3.5 \mathrm{keV})$ image. This size of detect cell optimizes the signal-to-noise ratio and detection sensitivity for on-axis point sources for the Einstein broad energy band. Background counts were estimated using a detect cell of the same size and position on the broad-band background map, which was generated by the standard processing software for each image by combining appropriate proportions of the instrument flat field (DSMAP) and the background light due to diffuse $\mathrm{X}$-rays (BEMAP; Harnden et al. 1984). Observations were determined to be detections when

$$
N_{\text {box }}>3 \sqrt{B_{\text {box }}},
$$

where $N_{\text {box }}=$ net counts (image counts minus background map counts) and $B_{\text {box }}=$ background map counts in the detection box and assuming the noise in the background map is negligible.

A best estimate or $3 \sigma$ upper limit on the source counts was determined using a circle with $3^{\prime}$ radius centered on the X-ray centroid (X-ray detections) or optical position (X-ray nondetections). This size circle ensures that the majority of counts are included and is appropriate for use with the standard effective area calibration (Harnden et al. 1984). Background was estimated using the same $3^{\prime}$ circle at the same position on the background map. Net source counts evaluated this way are given in Table 2. The error on the net counts was estimated as

$$
\sigma_{\text {cir }}=\sqrt{T_{\text {cir }}}
$$

where $T_{\text {cir }}=$ total counts in a $3^{\prime}$ circle, and we have assumed that the uncertainty in the background subtraction is negligible. The different expressions appropriate for detection and flux determination along with the use of differently sized regions in the two cases lead to the possibility that a source which gives a $3 \sigma$ detection may have a flux which is known less accurately. This situation arises for 57 of our observations (corresponding to 56 different quasars), which are treated as positive detections but are flagged in Table 2 (marked as 9 in the Note column) and Table 3 (marked as 3 in the Note column). Moreover, in a few cases (10) the detection process resulted in a large $(>3 \sigma)$ negative net rate for reasons not well understood (although seven of these 10 cases are likely due to the presence of a nearby contaminating source (cf. $\S 3.2$ be- 
TABLE 1

QUASARS AND SEYFERT 1 GaLAXIES INCLUDED IN DATABASE

\begin{tabular}{|c|c|c|c|c|c|c|c|c|c|c|c|}
\hline Name & Other Names & & R.A & A. (B1 & 950) & Dec. & & $\operatorname{Ref}$ & $\mathbf{Z}$ & Note & Sample \\
\hline $0002-422$ & & 0 & 2 & 15.9 & -42 & 14 & 7.0 & HB89 & 2.758 & 1 & \\
\hline $0003+158$ & PHL 658,4C 15.01,PKS,OB 106 & 0 & 3 & 25.1 & 15 & 53 & 7.4 & HB89 & .450 & 1 & PG \\
\hline $0007-000$ & UM 208 & 0 & 7 & 42.8 & 0 & -4 & 12.0 & HB89 & 2.31 & 1 & \\
\hline $0007+106$ & III $\mathrm{ZW} 2, \mathrm{PG}$ & 0 & 7 & 56.7 & 10 & 41 & 47.8 & HB89 & .089 & 1 & $P G$ \\
\hline $0007+171$ & $\mathrm{PKS}, 4 \mathrm{C} 17.04,0 \mathrm{~B} 113, \mathrm{MC} 3$ & 0 & 7 & 59.4 & 17 & 7 & 38.0 & HB89 & 1.601 & & \\
\hline 0009-016 & UM 211, РB 5791 & 0 & 9 & 37.2 & -1 & 38 & 48.0 & HB89 & 2.00 & 1 & \\
\hline 0013-004 & UM 224,PB 5829 & 0 & 13 & 28.7 & 0 & -29 & 5.0 & vV87 & 2.09 & 1 & \\
\hline $0014+166$ & $P G$ & 0 & 14 & 16.0 & 16 & 41 & 57.0 & HB89 & .100 & 1 & \\
\hline $0014+318$ & & 0 & 14 & 24.7 & 31 & 52 & 12.0 & B2 & 1.086 & 1 & \\
\hline $0014+159$ & & 0 & 14 & 35.4 & 15 & 59 & 4.0 & ANDP & 2.20 & 1 & \\
\hline $0015+155$ & & 0 & 15 & 54.8 & 15 & 35 & 48.0 & HB89 & 2.30 & 1 & \\
\hline $0016+731$ & S5 & 0 & 16 & 54.2 & 73 & 10 & 51.5 & HB89 & 1.781 & & \\
\hline $0017+154$ & 3CR $9,4 \mathrm{C} \quad 15.02, \mathrm{PHL} 2871,0 \mathrm{~B} \quad 129, \mathrm{MC} 3$ & 0 & 17 & 49.8 & 15 & 24 & 16.5 & HB89 & 2.012 & & $3 \mathrm{CR}$ \\
\hline $0019+011$ & UM $232, \mathrm{~PB} 5901$ & 0 & 19 & 53.6 & 1 & 7 & 35.0 & HB89 & 2.121 & 1 & \\
\hline $0026+129$ & PG & 0 & 26 & 38.1 & 12 & 59 & 29.6 & HB89 & .142 & 1 & PG \\
\hline $0037-018$ & UM 264 & 0 & 37 & 44.8 & -1 & 53 & 50.0 & HB89 & 2.34 & 1 & \\
\hline $0038-020$ & PKS, PB 6091 & 0 & 38 & 23.8 & -2 & 2 & 54.0 & HB89 & 1.178 & & \\
\hline $0043+008$ & UM 275,PHL 6612 & 0 & 43 & 39.5 & 0 & 48 & 3.0 & HB89 & 2.143 & 1 & \\
\hline $0044+030$ & PKS,PG,PHL 828 & 0 & 44 & 31.2 & 3 & 3 & 35.0 & HB89 & .624 & 1 & PG \\
\hline $0049+171$ & Mkn 1148 & 0 & 49 & 16.5 & 17 & 9 & 41.0 & SG83 & .064 & 1 & PG \\
\hline $0049+007$ & UM 287, PHL 868 & 0 & 49 & 28.4 & 0 & 45 & 13.0 & HB89 & 2.27 & 1 & \\
\hline $0049+014$ & UM 288 & 0 & 49 & 59.5 & 1 & 24 & 24.0 & HB89 & 2.31 & 1 & \\
\hline $0050+124$ & I $\mathrm{ZW} 1, \mathrm{PG}$ & 0 & 50 & 57.8 & 12 & 25 & 20.0 & HB89 & .061 & 1 & PG \\
\hline $0051+291$ & 4C $29.01, \mathrm{~B} 2$ & 0 & 51 & 2.1 & 29 & 8 & 51.2 & HB89 & 1.828 & & \\
\hline $0051+146$ & PHL 891 & 0 & 51 & 57.1 & 14 & 39 & 14.1 & HB89 & .874 & 1 & \\
\hline $0052+145$ & PHL 892 & 0 & 52 & 6.2 & 14 & 30 & 31.4 & HB89 & .911 & 1 & \\
\hline $0052+251$ & PG & 0 & 52 & 11.1 & 25 & 9 & 24.0 & HB89 & .155 & 1 & PG \\
\hline $0054+144$ & PHL 909 & 0 & 54 & 31.9 & 14 & 29 & 58.6 & HB89 & .171 & 1 & \\
\hline $0055+004$ & UM 294 & 0 & 55 & 50.8 & 0 & 25 & 3.0 & HB89 & 1.92 & 1 & \\
\hline $0056-001$ & PHL $923,4 \mathrm{C}-00.06, \mathrm{PKS}, 0 \mathrm{~B}-094$ & 0 & 56 & 31.7 & 0 & -9 & 19.2 & HB89 & .717 & & \\
\hline $0057+315$ & Mkn 352 & 0 & 57 & 9.1 & 31 & 33 & 28.0 & VV87 & .015 & 1 & \\
\hline $0058+019$ & PHL 938,UM 297 & 0 & 58 & 19.7 & 1 & 55 & 28.0 & HB89 & 1.955 & 1 & \\
\hline $0100+130$ & PHL 957 & 1 & 0 & 33.4 & 13 & 0 & 10.6 & HB89 & 2.681 & 1 & \\
\hline $0106+013$ & PKS, 4C $01.02,0 \mathrm{C} \quad 012, \mathrm{~PB} 6280$ & 1 & 6 & 4.5 & 1 & 19 & 1.4 & HB89 & 2.107 & & \\
\hline $0109+022$ & UM 87 & 1 & 9 & 42.9 & 2 & 14 & 0.0 & HB89 & 2.35 & 1 & \\
\hline $0112-017$ & PKS,UM 310,PB 6342 & 1 & 12 & 43.9 & -1 & 42 & 54.8 & HB89 & 1.365 & 1,3 & \\
\hline $0115-011$ & UM 314, PB 6370 & 1 & 15 & 54.7 & -1 & 8 & 24.0 & HB89 & 2.19 & 1 & \\
\hline $0117+213$ & PG & 1 & 17 & 34.7 & 21 & 18 & 4.0 & HB89 & 1.493 & 1 & PG \\
\hline $0119+041$ & OC $033, \mathrm{PKS}, \mathrm{GC}$ & 1 & 19 & 21.4 & 4 & 6 & 44.0 & HB89 & .637 & & \\
\hline 0119-013 & II $\mathrm{ZW} 1$ & 1 & 19 & 26.5 & -1 & 18 & 5.0 & HB89 & .054 & 1 & \\
\hline 0119-046 & PKS, 4C-04.04,0C-034,PB 8761 & 1 & 19 & 56.0 & -4 & 37 & 7.2 & HB89 & 1.948 & & \\
\hline $0119+229$ & $\mathrm{PG}$ & 1 & 19 & 57.0 & 22 & 54 & 35.0 & SG83 & .053 & 1 & PG \\
\hline $0121-590$ & ESO $113-$, IG $45, F 9$ & 1 & 21 & 51.2 & -59 & 3 & 58.9 & HB89 & .045 & 1 & \\
\hline $0122-380$ & & 1 & 22 & 2.2 & -38 & 0 & 4.0 & HB89 & 2.181 & 1 & \\
\hline $0126+030$ & UM 104, PB 6465 & 1 & 26 & 8.5 & 3 & 1 & 19.0 & HB89 & 1.62 & 1 & \\
\hline $0128+074$ & PHL 3375 ,PB 6482 & 1 & 28 & 25.2 & 7 & 28 & 14.5 & HB89 & .390 & 1 & \\
\hline $0130+033$ & PHL 1027, UM 114 & 1 & 30 & 31.6 & 3 & 23 & 41.3 & HB89 & .363 & 1 & \\
\hline $0130-403$ & & 1 & 30 & 50.5 & -40 & 21 & 54.0 & HB89 & 3.03 & 1 & \\
\hline $0130-406$ & & 1 & 30 & 50.7 & -40 & 38 & 13.0 & HB89 & 2.39 & 1 & \\
\hline $0131-404 A$ & & 1 & 31 & 3.5 & -40 & 27 & 23.0 & VV87 & 1.48 & 1 & \\
\hline $0131+037$ & PHL 1033, UM 118 & 1 & 31 & 7.8 & 3 & 42 & 13.5 & HB89 & .255 & 1 & \\
\hline $0131-409 B$ & & 1 & 31 & 40.0 & -40 & 54 & 23.0 & HB89 & 2.36 & 1 & \\
\hline $0131-409 A$ & & 1 & 31 & 48.4 & -40 & 54 & 49.0 & HB89 & 1.34 & 1 & \\
\hline $0132-408$ & & 1 & 32 & 20.8 & -40 & 48 & 26.0 & HB89 & 2.42 & 1 & \\
\hline $0133+207$ & 3CR 47,4C 20.07,PKS,CTA 14 & 1 & 33 & 40.4 & 20 & 42 & 10.6 & HB89 & .425 & & 3CR \\
\hline $0134+033$ & PHL 1070 & 1 & 34 & 43.3 & 3 & 23 & 14.0 & HB89 & .079 & 1 & \\
\hline
\end{tabular}




\begin{tabular}{|c|c|c|c|c|c|c|c|c|c|c|c|}
\hline Name & Other Names & & R.A & A. (B1 & 950) & Dec. & & Ref & $\mathrm{Z}$ & Note & Sample \\
\hline $0134+329$ & 3CR $48,4 \mathrm{C} 32.08$, NRAO $79,0 \mathrm{C} 358, \mathrm{DA} 5$ & 1 & 34 & 49.8 & 32 & 54 & 20.4 & HB89 & .367 & & 3CR \\
\hline $0135-247$ & PKS , 0C-259 & 1 & 35 & 17.2 & -24 & 46 & 9.4 & HB89 & .831 & & \\
\hline $0137+060$ & PHL 1092 & 1 & 37 & 19.0 & 6 & 4 & 10.5 & HB89 & .396 & 1 & \\
\hline $0137-010$ & NAB, UM 357, PHL 1096 & 1 & 37 & 43.8 & -1 & 5 & 12.9 & HB89 & .334 & 1 & \\
\hline 0140-306 & & 1 & 40 & 37.5 & -30 & 38 & 50.0 & HB89 & 3.13 & 1 & \\
\hline 0143-015 & UM 366, PHL 7756 & 1 & 43 & 18.2 & -1 & 35 & 30.0 & vV87 & 3.14 & 1 & \\
\hline 0143-010 & UM 368 & 1 & 43 & 46.7 & -1 & 1 & 26.0 & HB89 & 3.16 & 1 & \\
\hline $0145+042$ & UM 139 & 1 & 45 & 41.4 & 4 & 16 & 25.0 & vv87 & 2.03 & 1 & \\
\hline $0146+017$ & UM 141 & 1 & 46 & 44.0 & 1 & 42 & 30.0 & HB89 & 2.909 & 1 & \\
\hline $0149-397$ & & 1 & 49 & 18.3 & -39 & 42 & 42.7 & HB89 & 2.058 & 1 & \\
\hline $0151+045$ & PHL 1226 ,UM 145 & 1 & 51 & 51.6 & 4 & 33 & 37.7 & HB89 & .404 & 1 & \\
\hline $0157+001$ & Mkn 1014, PG & 1 & 57 & 15.8 & 0 & 9 & 10.0 & HB89 & .163 & 1 & PG \\
\hline $0159+036$ & UM 154, PB 6589 & 1 & 59 & 23.8 & 3 & 36 & 16.0 & HB89 & 2.44 & 1 & \\
\hline $0203+151 \mathrm{~A}$ & & 2 & 3 & 7.3 & 15 & 9 & 7.0 & HB89 & 2.00 & 1 & \\
\hline $0203+151 B$ & & 2 & 3 & 31.2 & 15 & 9 & 7.0 & ANDP & 2.5 & 1 & \\
\hline $0203+150$ & & 2 & 3 & 38.4 & 15 & 1 & 5.0 & HB89 & 2.10 & 1 & \\
\hline $0203+152$ & & 2 & 3 & 49.1 & 15 & 14 & 52.0 & ANDP & 2.30 & 1 & \\
\hline $0205+024$ & NAB, Mkn 586, PB 6679 & 2 & 5 & 14.5 & 2 & 28 & 42.7 & HB89 & .155 & 1 & \\
\hline 0205-379 & & 2 & 5 & 19.4 & -37 & 56 & 7.0 & HB89 & 2.41 & 1 & \\
\hline $0205+150$ & & 2 & 5 & 44.8 & 15 & 0 & 41.0 & HB89 & 2.40 & 1 & \\
\hline $0207-003$ & UM 402, $\mathrm{PB}$ 6709B & 2 & 7 & 17.0 & 0 & -19 & 6.0 & VV87 & 2.84 & 1 & \\
\hline 0207-398 & & 2 & 7 & 24.3 & -39 & 53 & 50.0 & HB89 & 2.813 & 1 & \\
\hline $0210+860$ & 3CR61.1BS0,RN 8 BS & 2 & 10 & 49.0 & 86 & 5 & 10.0 & HB89 & .184 & & \\
\hline 0212-009 & Mkn 590 & 2 & 12 & 0.4 & 0 & -59 & 57.0 & Vv87 & .027 & 1 & \\
\hline $0212+735$ & S5 & 2 & 12 & 50.0 & 73 & 35 & 40.5 & HB89 & 2.367 & & \\
\hline $0219+428$ & $3 \mathrm{C} 66 \mathrm{~A}$ & 2 & 19 & 30.0 & 42 & 48 & 30.4 & HB89 & .444 & & \\
\hline $0225+310$ & Mkn 1040 & 2 & 25 & 16.5 & 31 & 5 & 18.0 & Vv87 & .016 & 1 & \\
\hline $0225-014$ & PKS , 4C-01.11,0D-043 & 2 & 25 & 35.0 & -1 & 29 & 3.9 & HB89 & 2.037 & & \\
\hline $0226-038$ & PHL 1305, PKS , 4C-03.07, OD-044 & 2 & 26 & 22.1 & -3 & 50 & 59.5 & HB89 & 2.064 & & \\
\hline $0229+131$ & PKS, 4C 13.14, OD 148 & 2 & 29 & 2.4 & 13 & 9 & 41.0 & HB89 & 2.065 & & \\
\hline $0229+341$ & 3CR $68.1,4 \mathrm{C} 34.08$, NRA0 $105, \mathrm{DA} 78$ & 2 & 29 & 27.2 & 34 & 10 & 34.1 & HB89 & 1.238 & & \\
\hline $0232-090$ & NGC 985 & 2 & 32 & 10.5 & -9 & 0 & 21.0 & VV87 & .043 & 1 & \\
\hline $0234+285$ & $4 \mathrm{C} 28.07,0 \mathrm{D} 258, \mathrm{~B} 2, \mathrm{GC}$ & 2 & 34 & 55.6 & 28 & 35 & 8.0 & HB89 & 1.213 & & \\
\hline $0237-027$ & PKS & 2 & 37 & 13.7 & -2 & 47 & 32.5 & HB89 & 1.116 & & \\
\hline $0237+040$ & OD 062,PKS,GC & 2 & 37 & 14.3 & 4 & 3 & 17.0 & HB89 & .978 & & \\
\hline $0237-233$ & PKS,PHL 8462, OD-263 & 2 & 37 & 52.7 & -23 & 22 & 8.5 & HB89 & 2.223 & & \\
\hline $0238+069$ & Mkn 595 & 2 & 38 & 55.8 & 6 & 58 & 27.0 & VV87 & .028 & 1 & \\
\hline $0242-410$ & & 2 & 42 & 2.4 & -41 & 4 & 2.0 & HB89 & 2.214 & 1 & \\
\hline $0246+190$ & Mkn 372 & 2 & 46 & 31.4 & 19 & 5 & 50.0 & VV87 & .031 & 1 & \\
\hline $0254-404$ & & 2 & 54 & 39.1 & -40 & 24 & 59.0 & HB89 & 2.29 & 1 & \\
\hline $0306+169$ & & 3 & 6 & 6.7 & 16 & 54 & 23.0 & HB89 & 2.14 & 1 & \\
\hline $0307+169$ & 3C 79.0 & 3 & 7 & 11.5 & 16 & 54 & 37.0 & vV87 & .256 & 1 & \\
\hline $0312-770$ & PKS & 3 & 12 & 55.7 & -77 & 3 & 1.0 & HB89 & .223 & & \\
\hline $0333+321$ & NRAO $140,4 \mathrm{C} 32.14$, OE $355, \mathrm{~B} 2$ & 3 & 33 & 22.4 & 32 & 8 & 36.6 & HB89 & 1.258 & & \\
\hline 0336-019 & PKS,CTA 26,DA $110,0 \mathrm{E}-063$ & 3 & 36 & 58.9 & -1 & 56 & 16.3 & HB89 & .852 & & \\
\hline $0340+048$ & 3CR 93,PKS,OE $069,4 \mathrm{C} 04.13$ & 3 & 40 & 51.5 & 4 & 48 & 21.7 & HB89 & .357 & & \\
\hline $0400+258$ & OF $200, \mathrm{~B} 2, \mathrm{DW}, \mathrm{CTD} 26$ & 4 & 0 & 3.7 & 25 & 51 & 45.0 & HB89 & 2.109 & & \\
\hline $0403-132$ & PKS & 4 & 3 & 14.0 & -13 & 16 & 17.9 & HB89 & .571 & & \\
\hline $0405-123$ & MSH $04-12, \mathrm{PKS}, 0 \mathrm{~F}-109$ & 4 & 5 & 27.4 & -12 & 19 & 31.8 & HB89 & .574 & & \\
\hline $0409+229$ & 3C $108,4 \mathrm{C} 22.08$, NRAO 167, DA 128 & 4 & 9 & 44.7 & 22 & 57 & 27.8 & HB89 & 1.215 & & \\
\hline $0410+110$ & $3 C 109.0$ & 4 & 10 & 54.9 & 11 & 4 & 41.0 & VV87 & .306 & 1 & \\
\hline 0414-060 & $\mathrm{PKS}, 4 \mathrm{C}-05.17,3 \mathrm{C} 110,0 \mathrm{~F}-024$ & 4 & 14 & 49.2 & -6 & 1 & 4.3 & HB89 & .781 & & \\
\hline $0415+379$ & $3 \mathrm{C} 111.0$ & 4 & 15 & 0.6 & 37 & 54 & 19.0 & vV87 & .048 & 1 & \\
\hline $0418-550$ & NGC 1566 & 4 & 18 & 53.3 & -55 & 3 & 23.0 & VV87 & .004 & 1 & \\
\hline $0420-388$ & & 4 & 20 & 30.1 & -38 & 51 & 50.4 & HB89 & 3.12 & 1 & \\
\hline 0420-014 & PKS , 0F-035,0A 129 & 4 & 20 & 43.5 & -1 & 27 & 28.5 & HB89 & .915 & & \\
\hline $0424-131$ & PKS, NRAO $178,0 \mathrm{~F}-141.3$ & 4 & 24 & 47.8 & -13 & 9 & 33.4 & HB89 & 2.165 & & \\
\hline $0430+052$ & $3 \mathrm{C} 120$ & 4 & 30 & 31.6 & 5 & 14 & 59.0 & VV87 & .033 & 1 & \\
\hline $0434-104$ & Mkn 618 & 4 & 34 & 0.0 & -10 & 28 & 36.0 & VV87 & .035 & 1 & \\
\hline
\end{tabular}




\begin{tabular}{|c|c|c|c|c|c|c|c|c|c|c|c|}
\hline Name & Other Names & & & A. (B1S & 950) & Dec. & & Ref & $\mathrm{z}$ & Note & Sample \\
\hline $0438-166$ & & 4 & 38 & 11.6 & -16 & 38 & 12.0 & HB89 & 1.96 & 1 & \\
\hline $0438-436$ & PKS & 4 & 38 & 43.2 & -43 & 38 & 54.0 & HB89 & 2.852 & & \\
\hline $0439-164$ & & 4 & 39 & 34.9 & -16 & 24 & 24.0 & ANDP & 2.20 & 1 & \\
\hline $0440-003$ & PKS, NRAO 190, OF-067,DA 145 & 4 & 40 & 5.3 & 0 & -23 & 20.6 & HB89 & .844 & & \\
\hline 0454-234 & PKS0454-234 & 4 & 54 & 57.3 & -23 & 29 & 29.0 & VV87 & 1.009 & 1 & \\
\hline $0458-020$ & PKS,DA $157,4 C-02.19,0 F-098$ & 4 & 58 & 41.3 & -2 & 3 & 34.5 & HB89 & 2.286 & & \\
\hline 0513-002 & AKN 120 & & 13 & 37.9 & 0 & -12 & 16.0 & vV87 & .033 & 1 & \\
\hline $0518+165$ & 3CR $138,4 \mathrm{C} \quad 16.12, \mathrm{PKS}$, OG 130.2 & 5 & 18 & 16.5 & 16 & 35 & 26.2 & HB89 & .759 & & $3 \mathrm{CR}$ \\
\hline $0518-458$ & PKS $0518-45$ & 5 & 18 & 18.2 & -45 & 49 & 48.0 & VV87 & .034 & 1 & \\
\hline $0528-250$ & PKS & & 28 & 5.2 & -25 & 5 & 45.0 & VV87 & 2.765 & & \\
\hline $0537-441$ & PKS & & 37 & 21.1 & -44 & 6 & 45.4 & HB89 & .894 & & \\
\hline $0537-286$ & PKS , OG-263,B1 & & 37 & 56.9 & -28 & 41 & 28.3 & HB89 & 3.11 & & \\
\hline $0538+498$ & 3CR $147,4 \mathrm{C} 49.14, \mathrm{NRAO} 221, \mathrm{OG} 465, \mathrm{DA}$ & 5 & 38 & 43.6 & 49 & 49 & 42.8 & HB89 & .545 & & $3 \mathrm{CR}$ \\
\hline 0605-085 & $\mathrm{OH}-010$ & 6 & 5 & 36.0 & -8 & 34 & 19.0 & HB89 & .87 & & \\
\hline $0607-157$ & $\mathrm{PKS}, \mathrm{MC}$ & 6 & 7 & 26.1 & -15 & 42 & 4.2 & HB89 & .324 & & \\
\hline $0637-752$ & PKS , MC & 6 & 37 & 23.3 & -75 & 13 & 37.8 & HB89 & .656 & & \\
\hline $0642+449$ & $\mathrm{OH} 471$ & 6 & 42 & 53.0 & 44 & 54 & 31.1 & HB89 & 3.402 & & \\
\hline $0655+542$ & Mkn 374 & 6 & 55 & 34.5 & 54 & 15 & 57.0 & VV87 & .044 & 1 & \\
\hline $0710+118$ & 3CR $175,4 \mathrm{C} 11.26, \mathrm{PKS}, \mathrm{OI} 117$ & 7 & 10 & 15.4 & 11 & 51 & 23.9 & HB89 & .768 & & $3 \mathrm{CR}$ \\
\hline $0730+659$ & W1 & 7 & 30 & 18.0 & 65 & 59 & 39.0 & HB89 & 1.937 & & \\
\hline $0732+588$ & Mkn 9 & 7 & 32 & 42.2 & 58 & 52 & 56.0 & VV87 & .039 & 1 & \\
\hline $0736+017$ & PKS,OI 061 & 7 & 36 & 42.5 & 1 & 44 & 0.1 & HB89 & .191 & & \\
\hline $0738+313$ & OI $363, \mathrm{~B} 2, \mathrm{DW}$ & 7 & 38 & 0.2 & 31 & 19 & 1.8 & HB89 & .631 & & \\
\hline $0740+380$ & 3CR $186,4 \mathrm{C} 38.21$, NRAO 273, OI 368 & 7 & 40 & 56.8 & 38 & 0 & 31.0 & HB89 & 1.063 & & $3 \mathrm{CR}$ \\
\hline $0752+393$ & Mkn 382 & 7 & 52 & 3.6 & 39 & 19 & 7.0 & VV87 & .034 & 1 & \\
\hline $0758+143$ & 3CR 190, PKS, 4C 14.25, NRAO 278 & 7 & 58 & 45.1 & 14 & 23 & 4.3 & HB89 & 1.195 & & \\
\hline $0802+103$ & 3CR $191,4 \mathrm{C} 10.25, \mathrm{PKS}, 0 \mathrm{~J} 103.3$ & 8 & 2 & 3.8 & 10 & 23 & 56.3 & HB89 & 1.956 & & $3 \mathrm{CR}$ \\
\hline $0804+761$ & PG & 8 & 4 & 35.4 & 76 & 11 & 32.0 & HB89 & .100 & 1 & PG \\
\hline $0805+046$ & 4C $05.34,0 \mathrm{~J} 008$ & 8 & 5 & 19.2 & 4 & 41 & 20.5 & HB89 & 2.877 & & \\
\hline $0805+047$ & KP 2 & 8 & 5 & 29.0 & 4 & 45 & 31.0 & HB89 & 2.06 & 1 & \\
\hline $0809+483$ & 3CR $196,4 \mathrm{C} 48.22, \mathrm{CTA} 45,0 \mathrm{~J} 417, \mathrm{DA} 2$ & 8 & 9 & 59.4 & 48 & 22 & 7.7 & HB89 & .871 & & $3 \mathrm{CR}$ \\
\hline $0824+110$ & MC 5 & 8 & 24 & 22.4 & 11 & 2 & 19.4 & HB89 & 2.278 & & \\
\hline $0827+243$ & OJ $248, A 0, B 2$ & 8 & 27 & 54.4 & 24 & 21 & 7.7 & HB89 & .939 & & \\
\hline $0833+654$ & 3CR $204,4 \mathrm{C} 65.09$, NRAO 297 & 8 & 33 & 18.0 & 65 & 24 & 4.4 & HB89 & 1.112 & & $3 C R$ \\
\hline 0834-201 & PKS & 8 & 34 & 24.6 & -20 & 6 & 30.1 & HB89 & 2.752 & & \\
\hline $0834+654$ & & 8 & 34 & 25.4 & 65 & 29 & 21.0 & ANDP & 2.2 & 1 & \\
\hline $0835+580$ & 3CR $205,4 \mathrm{C} 58.16,0 \mathrm{~J} 558, \mathrm{NRAO} 298, \mathrm{DA}$ & 8 & 35 & 10.0 & 58 & 4 & 51.8 & HB89 & 1.534 & & $3 \mathrm{CR}$ \\
\hline $0835+583$ & & 8 & 35 & 32.0 & 58 & 19 & 8.0 & ANDP & 3.00 & 1 & \\
\hline $0836+654$ & & 8 & 36 & 37.5 & 65 & 24 & 1.0 & HB89 & 1.9 & 1 & \\
\hline $0837-120$ & 3C 206, PKS,NRAO $299,0 \mathrm{~J}-162$ & 8 & 37 & 28.0 & -12 & 3 & 54.2 & HB89 & .198 & & \\
\hline $0838+133 A$ & 3CR $207,4 \mathrm{C} 13.38, \mathrm{PKS}, 0 \mathrm{~J} 163$ & 8 & 38 & 1.7 & 13 & 23 & 5.6 & HB89 & .684 & & $3 \mathrm{CR}$ \\
\hline $0838+770$ & VII ZW 244 & 8 & 38 & 32.0 & 77 & 3 & 59.0 & SG83 & .131 & 1 & PG \\
\hline $0838+131$ & & 8 & 38 & 48.4 & 13 & 10 & 0.0 & HB89 & 1.88 & 1 & \\
\hline $0838+133 B$ & & 8 & 38 & 50.5 & 13 & 19 & 42.01 & HB89 & 1.80 & 1 & \\
\hline $0843+161$ & & 8 & 43 & 2.0 & 16 & 8 & 39.71 & HB89 & .863 & 1 & \\
\hline $0843+349$ & & 8 & 43 & 50.6 & 34 & 59 & 27.2 & HB89 & 1.575 & & \\
\hline $0844+349$ & PG & 8 & 44 & 33.9 & 34 & 56 & $9.0 s$ & SG83 & .064 & 1,4 & \\
\hline $0848+155$ & LB $8755,0 \mathrm{~J} 180$ & 8 & 48 & 4.5 & 15 & 33 & $31.4 \mathrm{H}$ & HB89 & 2.009 & 1 & \\
\hline $0848+163$ & LB 8775 & 8 & 48 & 53.7 & 16 & 23 & 40.0 & vV87 & 1.932 & 1 & \\
\hline $0850+140$ & 3CR $208,4 \mathrm{C} 14.28, \mathrm{PKS}, 0 \mathrm{~J} 184$ & 85 & 50 & 22.8 & 14 & 3 & 58.3 & HB89 & 1.11 & & $3 \mathrm{CR}$ \\
\hline $0855+188$ & LB 8991 & 85 & 55 & 40.2 & 18 & 48 & 48.4 & HB89 & 1.013 & 1 & \\
\hline $859-140$ & PKS, 0J-199, MSH 09-11 & 85 & 59 & 55.0 & -14 & 3 & 38.9 & HB89 & 1.327 & & \\
\hline $903+169$ & 3CR $215,4 \mathrm{C} \quad 16.26, \mathrm{PKS}, \mathrm{LB} 9308$ & 9 & 3 & 44.2 & 16 & 58 & 15.7 & HB89 & .411 & & $3 C R$ \\
\hline $906+430$ & 3CR $216,4 \mathrm{C} 43.17$, NRAO 317, OK $410, \mathrm{DA}$ & 9 & 6 & 17.3 & 43 & 5 & 59.01 & HB89 & .67 & & \\
\hline $906+015$ & PKS, 4C 01.24, DA $263,0 \mathrm{~K} 011$ & 9 & 6 & 35.2 & 1 & 33 & 48.21 & HB89 & 1.018 & & \\
\hline $906+484$ & PG & 9 & 6 & 45.3 & 48 & 25 & $55.8 \mathrm{l}$ & HB89 & .118 & 1 & \\
\hline $910+403$ & NGC2782 U1 & 91 & 10 & 54.0 & 40 & 19 & 18.0 & vV87 & .936 & 1 & \\
\hline $915+165$ & Mkn 704 & 91 & 15 & 39.4 & 16 & 30 & $59.0 \mathrm{~V}-\mathrm{r}-\mathrm{r}-\mathrm{r}-\mathrm{r}$ & VV87 & .029 & 1 & \\
\hline $923+201$ & PG,TON 1057 & 92 & 23 & 5.8 & 20 & 7 & $7.0 \mathrm{~F}$ & HB89 & .190 & 1 & PG \\
\hline
\end{tabular}




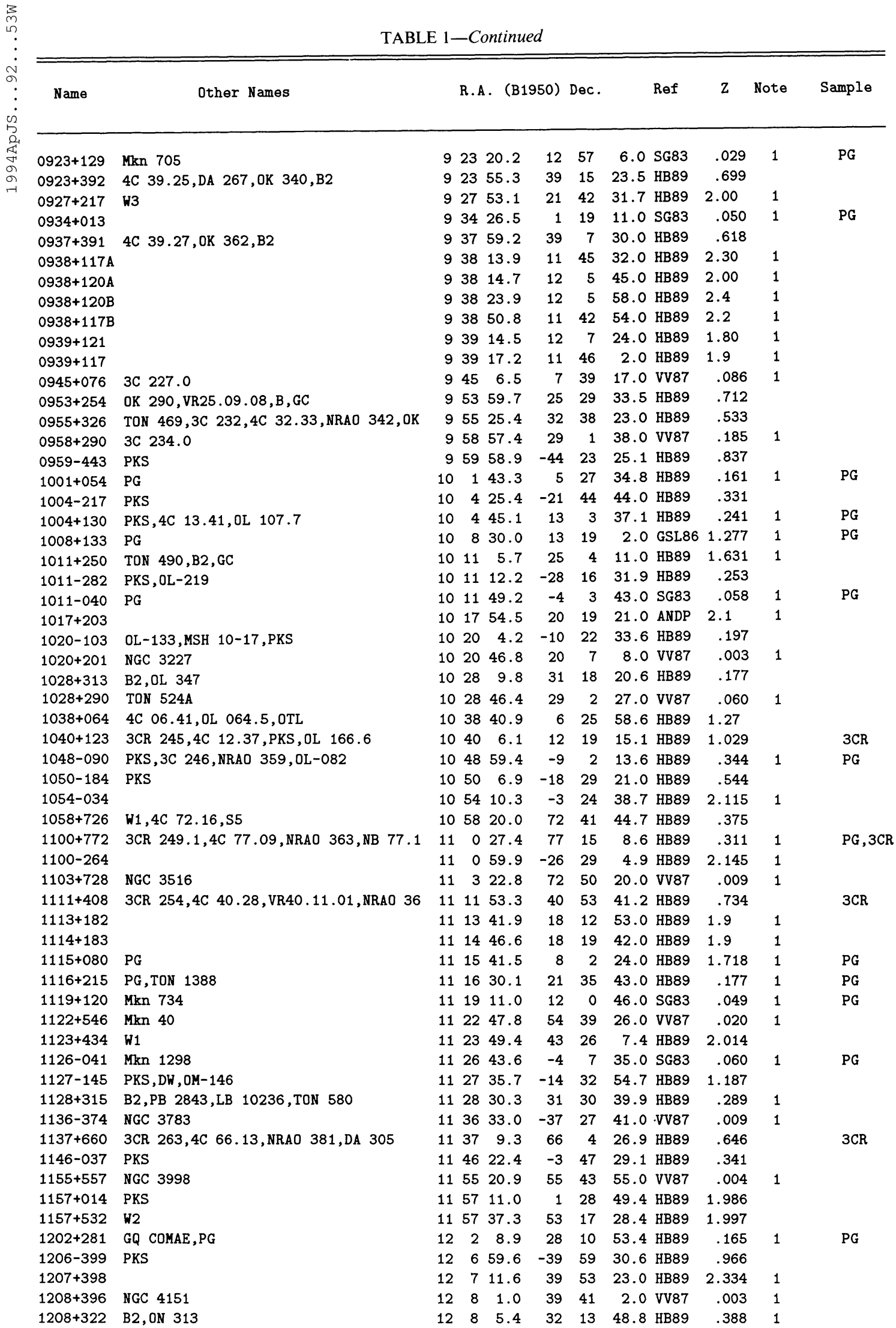




\begin{tabular}{|c|c|c|c|c|c|c|c|c|c|c|c|}
\hline Name & Other Names & & R.A & 1. (B19 & 50) & Dec. & & $\operatorname{Ref}$ & $\mathrm{Z}$ & Note & Sample \\
\hline $1208+398$ & & 12 & 8 & 39.9 & 39 & 50 & 2.0 & ANDP & 1.5 & 1 & \\
\hline $1209+107 \mathrm{~A}$ & KP 9 & 12 & 9 & 8.4 & 10 & 46 & 58.1 & HB89 & 2.191 & 1 & \\
\hline $1209+107 \mathrm{~B}$ & KP 10 & 12 & 9 & 12.2 & 10 & 42 & 23.0 & HB89 & 1.9 & 1 & \\
\hline $1209+109$ & KP 11 & 12 & 9 & 56.7 & 10 & 58 & 4.1 & HB89 & 2.1 & 1 & \\
\hline $1211+143$ & PG & 12 & 11 & 44.8 & 14 & 19 & 53.0 & SG83 & .085 & 1 & PG \\
\hline $1214+074$ & NGC 4235 & 12 & 14 & 36.7 & 7 & 28 & 9.0 & VV87 & .007 & 1 & \\
\hline $1215+300$ & Mkn 766 & 12 & 15 & 55.6 & 30 & 5 & 26.0 & vV87 & .012 & 1 & \\
\hline $1216+069$ & PG & 12 & 16 & 47.2 & 6 & 55 & 19.0 & HB89 & .334 & 1 & PG \\
\hline $1217+023$ & PKS,ON 029, UM 492 & 12 & 17 & 38.4 & 2 & 20 & 20.9 & HB89 & .24 & & \\
\hline $1218+339$ & 3CR $270.1,4 \mathrm{C} 33.29$, NRAO 396, ON 330 & 12 & 18 & 3.9 & 33 & 59 & 50.1 & HB89 & 1.519 & 1 & \\
\hline $1219+755$ & Mkn 205 & 12 & 19 & 33.5 & 75 & 35 & 14.6 & HB89 & .070 & 1 & \\
\hline $1219+044$ & PKS & 12 & 19 & 48.4 & 4 & 29 & 59.0 & HB89 & .965 & & \\
\hline $1221+758$ & W1 & 12 & 21 & 21.3 & 75 & 53 & 6.1 & HB89 & 1.632 & & \\
\hline $1222+226$ & & 12 & 22 & 47.2 & 22 & 37 & 4.0 & VV87 & 2.29 & 1 & \\
\hline $1222+228 B$ & KP 13 & 12 & 22 & 52.9 & 22 & 48 & 4.0 & HB89 & 1.87 & 1 & \\
\hline $1222+228 \mathrm{~A}$ & TON $1530, P G$ & 12 & 22 & 56.6 & 22 & 51 & 49.0 & VV87 & 2.051 & 1 & PG \\
\hline $1223+252$ & TON $616,4 \mathrm{C} 25.40, \mathrm{VR} 25.12 .02, \mathrm{ON} 239$ & 12 & 23 & 9.1 & 25 & 15 & 11.9 & HB89 & .268 & & \\
\hline $1223+227$ & $\mathrm{KP} 14$ & 12 & 23 & 56.2 & 22 & 45 & 0.0 & HB89 & 1.93 & 1 & \\
\hline $1225+317$ & B2 & 12 & 25 & 55.9 & 31 & 45 & 12.6 & HB89 & 2.219 & & \\
\hline $1226+023$ & 3CR $273,4 \mathrm{C} 02.32$, NRAO $400,0 \mathrm{~N} 044$,DA & 12 & 26 & 33.2 & 2 & 19 & 43.2 & HB89 & .158 & 1 & $\mathrm{PG}, 3 \mathrm{CR}$ \\
\hline $1228+078$ & $\mathrm{KP} 16$ & 12 & 28 & 2.4 & 7 & 50 & 21.8 & HB89 & 1.813 & 1 & \\
\hline $1228+076$ & KP 17 & 12 & 28 & 35.3 & 7 & 41 & 12.1 & HB89 & 1.878 & 1 & \\
\hline $1228+077$ & $\mathrm{KP} 18$ & 12 & 28 & 47.9 & 7 & 42 & 31.0 & HB89 & 2.391 & 1 & \\
\hline $1229+078 \mathrm{~A}$ & $1229.0+07.8$ & 12 & 29 & 3.2 & 7 & 51 & 15.0 & VV87 & 1.93 & 1 & \\
\hline $1229+116$ & & 12 & 29 & 12.4 & 11 & 37 & 48.0 & HB89 & 1.80 & 1 & \\
\hline $1229-021$ & PKS , 4C-02.55, 0N-049 & 12 & 29 & 25.9 & -2 & 7 & 32.5 & HB89 & 1.038 & & \\
\hline $1229+204$ & TON 1542, Mkn 771 AGN, AKN 374 & 12 & 29 & 33.1 & 20 & 26 & 2.7 & HB89 & .064 & 1 & PG \\
\hline $1229+078 B$ & $\mathrm{KP} 20$ & 12 & 29 & 37.7 & 7 & 50 & 17.2 & HB89 & 1.51 & 1 & \\
\hline $1229+077$ & KP 21 & 12 & 29 & 49.5 & 7 & 47 & 8.0 & VV87 & 2.76 & 1 & \\
\hline $1230+120$ & & 12 & 30 & 11.0 & 12 & 2 & 59.0 & HB89 & 1.90 & 1 & \\
\hline $1235+264$ & WEE 73 & 12 & 35 & 25.1 & 26 & 27 & 30.0 & HB89 & 2.50 & 1 & \\
\hline $1237-101$ & $\mathrm{ON}-162, \mathrm{PKS}$ & 12 & 37 & 7.3 & -10 & 7 & 0.4 & HB89 & .753 & & \\
\hline $1241+166$ & 3CR $275.1,4 \mathrm{C} 16.34, \mathrm{PKS}, \mathrm{ON} 169$ & 12 & 41 & 27.6 & 16 & 39 & 18.0 & HB89 & .557 & 1 & \\
\hline $1241+176$ & PG & 12 & 41 & 41.0 & 17 & 37 & 29.0 & HB89 & 1.273 & 1 & PG \\
\hline $1243+346$ & KP 22 & 12 & 43 & 45.3 & 34 & 37 & 25.0 & HB89 & 2.29 & 1 & \\
\hline $1244+026$ & PG & 12 & 44 & 2.1 & 2 & 38 & 31.0 & SG83 & .048 & 1 & PG \\
\hline $1244+345$ & KP 23 & 12 & 44 & 5.3 & 34 & 33 & 5.0 & HB89 & 1.96 & 1 & \\
\hline $1244+346 \mathrm{~A}$ & KP 24 & 12 & 44 & 8.8 & 34 & 41 & 3.8 & HB89 & 1.9 & 1 & \\
\hline $1244+346 B$ & KP 25 & 12 & 44 & 14.5 & 34 & 40 & 54.8 & HB89 & 2.3 & 1 & \\
\hline $1244+347$ & KP 26 & 12 & 44 & 57.3 & 34 & 43 & 54.1 & HB89 & 2.48 & 1 & \\
\hline $1245+345$ & B $19, A B 7$ & 12 & 45 & 3.2 & 34 & 31 & 31.4 & HB89 & 2.065 & 1 & \\
\hline $1245+343$ & KP 27 & 12 & 45 & 20.4 & 34 & 18 & 27.0 & HB89 & 1.82 & 1 & \\
\hline $1245+342$ & KP 28 & 12 & 45 & 39.3 & 34 & 16 & 34.0 & HB89 & 2.07 & 1 & \\
\hline $1246+344$ & KP 29 & 12 & 46 & 5.6 & 34 & 29 & 0.0 & HB89 & 2.23 & 1 & \\
\hline $1246+377$ & BSO $1, \mathrm{AB} 9$ & 12 & 46 & 28.7 & 37 & 46 & 49.7 & HB89 & 1.242 & 1 & \\
\hline $1246+346$ & $\mathrm{KP} 30, \mathrm{~B} 46$ & 12 & 46 & 29.6 & 34 & 40 & 49.3 & HB89 & .271 & 1 & \\
\hline $1246-057$ & & 12 & 46 & 38.8 & -5 & 42 & 58.3 & HB89 & 2.236 & 1 & \\
\hline $1250+568$ & 3CR 277.1 ,4C 56.20 , NRAO 409 , ON 584 & 12 & 50 & 15.3 & 56 & 50 & 36.5 & HB89 & .321 & & $3 \mathrm{CR}$ \\
\hline $1252+119$ & PKS, ON 187, MC 2 & 12 & 52 & 7.7 & 11 & 57 & 21.1 & HB89 & .871 & & \\
\hline $1253+359$ & BF 8 & 12 & 53 & 32.1 & 35 & 56 & 42.0 & HB89 & 2.09 & 1 & $\mathrm{BF}$ \\
\hline $1253-055$ & 3C $279,4 \mathrm{C}-05.55,0 \mathrm{~N}-089, \mathrm{NRAO} 413, \mathrm{CTA}$ & 125 & 53 & 35.9 & -5 & 31 & 8.4 & HB89 & .538 & & \\
\hline $1253+358$ & $\mathrm{BF} 12$ & 12 & 53 & 38.6 & 35 & 48 & 43.0 & HB89 & .74 & 1 & $\mathrm{BF}$ \\
\hline $1253+361$ & BF 16 & 12 & 53 & 49.4 & 36 & 7 & 14.0 & HB89 & .69 & 1 & $\mathrm{BF}$ \\
\hline $1253+360$ & BF 17 & 12 & 53 & 49.8 & 36 & 5 & 45.0 & HB89 & 1.37 & 1 & $\mathrm{BF}$ \\
\hline $1254+571$ & Mkn 231 & 12 & 54 & 5.0 & 57 & 8 & 38.0 & VV87 & .041 & 1 & \\
\hline $1254+047$ & PG,PB 4381 & 12 & 54 & 27.6 & 4 & 43 & 47.0 & HB89 & 1.024 & 1 & PG \\
\hline $1254+356 \mathrm{~A}$ & BF 36 & 12 & 54 & 47.4 & 35 & 38 & 35.0 & VV87 & 1.35 & 1 & $\mathrm{BF}$ \\
\hline $1254+360$ & BF 46 & 12 & 54 & 56.2 & 36 & 5 & 37.0 & HB89 & 1.50 & 1 & $\mathrm{BF}$ \\
\hline $1255+359$ & BF 51 & 125 & 55 & 8.3 & 35 & 59 & 32.0 & HB89 & .53 & 1 & $\mathrm{BF}$ \\
\hline
\end{tabular}


TABLE 1-Continued

\begin{tabular}{|c|c|c|c|c|c|c|c|c|c|c|c|}
\hline Name & Other Names & & R.A & A. (B1S & 950) & Dec. & & Ref & $z$ & Note & Sample \\
\hline $1256+357$ & B 194, BF 71 & 12 & 56 & 7.9 & 35 & 44 & 52.0 & VV87 & 1.864 & 1 & $\mathrm{BF}$ \\
\hline $1256+355$ & BF 72 & 12 & 56 & 10.9 & 35 & 32 & 18.0 & HB89 & 1.75 & 1 & $\mathrm{BF}$ \\
\hline $1257+361$ & BF 92 & 12 & 57 & 6.1 & 36 & 7 & 29.0 & HB89 & .82 & 1 & $\mathrm{BF}$ \\
\hline $1257+359$ & BF 105 & 12 & 57 & 33.9 & 35 & 55 & 53.0 & HB89 & .324 & 1 & $\mathrm{BF}$ \\
\hline $1257+356$ & $\mathrm{BF} 112$ & 12 & 57 & 53.7 & 35 & 38 & 57.0 & HB89 & 1.79 & 1 & $\mathrm{BF}$ \\
\hline $1258+287$ & $5 \mathrm{C} 4.105, \mathrm{~A} 2316, \mathrm{~PB} 3207, \mathrm{US} 122$ & 12 & 58 & 4.3 & 28 & 46 & 17.0 & HB89 & .648 & & \\
\hline $1258+286 A$ & W 61972, A2 327, PB 3214 ,US 130 & 12 & 58 & 23.8 & 28 & 39 & 28.0 & HB89 & 1.922 & 1 & \\
\hline $1258+356$ & B $196, A B \quad 86, \mathrm{BF}^{2} 141$ & 12 & 58 & 41.7 & 35 & 38 & 44.0 & HB89 & .324 & 1,2 & $\mathrm{BF}$ \\
\hline $1258+343$ & B $471, \mathrm{AB} 87$ & 12 & 58 & 49.5 & 34 & 22 & 38.1 & HB89 & .774 & 1 & \\
\hline $1258+286 B$ & $5 \mathrm{C} 4.127$ & 12 & 58 & 56.1 & 28 & 37 & 42.7 & HB89 & 1.373 & & \\
\hline $1258+342$ & KP 33 & 12 & 58 & 59.6 & 34 & 16 & 32.0 & HB89 & 1.93 & 1 & \\
\hline $1259+357$ & BF 161 & 12 & 59 & 25.6 & 35 & 46 & 38.0 & HB89 & 2.04 & 1 & $\mathrm{BF}$ \\
\hline $1259+344 \mathrm{~A}$ & BSO $6, \mathrm{~B} 243, \mathrm{AB} 90$ & 12 & 59 & 30.9 & 34 & 27 & 8.8 & HB89 & 1.956 & 1 & \\
\hline $1259+361$ & BF 164 & 12 & 59 & 32.5 & 36 & 10 & 12.0 & HB89 & .69 & 1 & $\mathrm{BF}$ \\
\hline $1259+359$ & BF 166 & 12 & 59 & 39.1 & 35 & 55 & 50.0 & HB89 & 1.23 & 1 & $\mathrm{BF}$ \\
\hline $1259+344 B$ & KP 35 & 12 & 59 & 44.2 & 34 & 25 & 46.0 & HB89 & 2.82 & 1 & \\
\hline $1259+360$ & BF 170 & 12 & 59 & 47.7 & 36 & 3 & 23.0 & VV87 & 1.76 & 1 & $\mathrm{BF}$ \\
\hline $1300+360$ & BF 175 & 13 & 0 & 0.0 & 36 & 4 & 53.0 & HB89 & .68 & 1 & $\mathrm{BF}$ \\
\hline $1300-243$ & & 13 & 0 & 36.7 & -24 & 18 & 55.6 & HB89 & 2.259 & 1 & \\
\hline $1301+359$ & BF 216 & 13 & 1 & 29.4 & 35 & 54 & 24.0 & HB89 & 1.47 & 1 & $\mathrm{BF}$ \\
\hline $1301+361$ & BF 219 & 13 & 1 & 38.4 & 36 & 8 & 30.0 & VV87 & 1.71 & 1 & $\mathrm{BF}$ \\
\hline $1301+358 \mathrm{~A}$ & B 286, AB 109, BF 222 & 13 & 1 & 42.0 & 35 & 49 & 21.5 & HB89 & .327 & 1 & $\mathrm{BF}$ \\
\hline $1301+358 B$ & BF 225 & 13 & 1 & 49.8 & 35 & 52 & 56.0 & HB89 & .91 & 1 & $\mathrm{BF}$ \\
\hline $1301+356$ & BF 227 & 13 & 1 & 56.5 & 35 & 41 & 53.0 & HB89 & 2.04 & 1 & $\mathrm{BF}$ \\
\hline $1302+358$ & BF 230 & 13 & 2 & 4.3 & 35 & 49 & 30.0 & HB89 & 1.99 & 1 & $\mathrm{BF}$ \\
\hline $1302+357$ & B 288, AB 115, BF 237 & 13 & 2 & 17.1 & 35 & 45 & 11.4 & HB89 & 1.275 & 1,2 & $\mathrm{BF}$ \\
\hline $1302-102$ & PKS , 0P-106,PG & 13 & 2 & 55.8 & -10 & 17 & 16.7 & HB89 & .286 & 1 & PG \\
\hline $1303+355$ & BF 262 & 13 & 3 & 10.3 & 35 & 33 & 16.0 & VV87 & .97 & 1 & $\mathrm{BF}$ \\
\hline $1303+357 \mathrm{~A}$ & BF 263 & 13 & 3 & 11.9 & 35 & 44 & 5.0 & HB89 & 1.62 & 1 & $\mathrm{BF}$ \\
\hline $1303+362$ & BF 264 & 13 & 3 & 16.1 & 36 & 14 & 32.0 & HB89 & 1.35 & 1 & $\mathrm{BF}$ \\
\hline $1303+358$ & BF 270 & 13 & 3 & 28.6 & 35 & 48 & 58.0 & HB89 & .378 & 1,2 & $\mathrm{BF}$ \\
\hline $1303+360$ & BF 281 & 13 & 3 & 44.1 & 36 & 0 & 1.0 & HB89 & 2.43 & 1 & $\mathrm{BF}$ \\
\hline $1304+310$ & US 272 & 13 & 4 & 12.1 & 31 & 5 & 29.0 & HB89 & .422 & 1 & \\
\hline $1304+346$ & B 340, Mkn $64, \mathrm{AB} 133$ & 13 & 4 & 48.0 & 34 & 40 & 24.2 & HB89 & .189 & 1 & \\
\hline $1307+085$ & $P G$ & 13 & 7 & 16.2 & 8 & 35 & 47.0 & HB89 & .155 & 1 & PG \\
\hline $1309-056$ & & 13 & 9 & 0.7 & -5 & 36 & 43.4 & HB89 & 2.224 & 1 & \\
\hline $1310-108$ & II SZ 10 & 13 & 10 & 28.0 & -10 & 51 & 48.0 & SG83 & .035 & 1 & PG \\
\hline $1311+362$ & BSO $11, \mathrm{~B} 416, \mathrm{AB} 168$ & 13 & 11 & 19.5 & 36 & 15 & 40.7 & HB89 & 2.084 & 1 & \\
\hline $1316+179$ & & 13 & 16 & 58.4 & 17 & 58 & 22.0 & ANDP & 1.3 & 1 & \\
\hline $1318+290 \mathrm{~A}$ & TON 155, PB 3520 & 13 & 18 & 53.7 & 29 & 3 & 30.3 & HB89 & 1.703 & 1 & \\
\hline $1321+294$ & TON 157 & 13 & 21 & 0.0 & 29 & 25 & 45.0 & vV87 & .960 & 1 & \\
\hline $1328+254$ & $3 \mathrm{CR} 287,4 \mathrm{C} 25.43, \mathrm{NRAO} \quad 424$, OP $247, \mathrm{DA}$ & 13 & 28 & 15.9 & 25 & 24 & 37.4 & HB89 & 1.055 & & $3 \mathrm{CR}$ \\
\hline $1328+307$ & $3 \mathrm{CR} 286,4 \mathrm{C} 30.26$, NRAO 425, OP $348, \mathrm{DA}$ & 13 & 28 & 49.7 & 30 & 45 & 58.6 & HB89 & .849 & & $3 C R$ \\
\hline $1330+022$ & 3C 287.1 & 13 & 30 & 20.5 & 2 & 16 & 8.0 & vV87 & .215 & 1 & \\
\hline $1331+170$ & MC 3,PB 3977, OP 151 & 13 & 31 & 10.1 & 17 & 4 & 25.0 & HB89 & 2.081 & & \\
\hline $1332+552$ & 4C 55.27, OP 554, LB 685 & 13 & 32 & 15.8 & 55 & 16 & 45.6 & HB89 & 1.249 & & \\
\hline $1333+176$ & PG,PB 4007 & 13 & 33 & 36.7 & 17 & 40 & 31.0 & HB89 & .554 & 1 & PG \\
\hline $1333+286$ & RS 23 & 13 & 33 & 54.2 & 28 & 40 & 15.9 & HB89 & 1.908 & 1 & \\
\hline $1335-127$ & PK & 13 & 35 & 0.0 & -12 & 42 & 9.0 & vV87 & .541 & 1 & \\
\hline $1340+606$ & 3CR $288.1,4 \mathrm{C} 60.18,0 \mathrm{P} 668, \mathrm{NRAO} 428$ & 13 & 40 & 30.0 & 60 & 36 & 47.9 & HB89 & .961 & & $3 C R$ \\
\hline $1346-036$ & & 13 & 46 & 8.2 & -3 & 38 & 30.5 & HB89 & 2.344 & 1 & \\
\hline $1351+640$ & PG & 13 & 51 & 46.3 & 64 & 0 & 28.4 & HB89 & .088 & 1 & PG \\
\hline $1351+695$ & Mkn 279 & 13 & 51 & 53.6 & 69 & 33 & 13.0 & vV87 & .031 & 1 & \\
\hline $1352+183$ & PG,PB 4142 & 13 & 52 & 11.5 & 18 & 20 & 58.0 & HB89 & .152 & 1 & PG \\
\hline $1352+011$ & $P G$ & 13 & 52 & 25.8 & 1 & 6 & 50.0 & GSL86 & 1.117 & 1 & PG \\
\hline 1354-152 & PKS, OP-192 & 13 & 54 & 28.6 & -15 & 12 & 52.1 & HB89 & 1.89 & & \\
\hline $1355-416$ & PKS, MSH $\quad 13-45$ & 13 & 55 & 56.8 & -41 & 38 & 16.7 & HB89 & .313 & & \\
\hline $1358+043$ & $P G$ & 13 & 58 & 0.6 & 4 & 19 & 27.4 & HB89 & .427 & 1 & \\
\hline $1402-012$ & PKS, UM 632 & 14 & 2 & 11.3 & -1 & 16 & 1.9 & HB89 & 2.518 & & \\
\hline
\end{tabular}




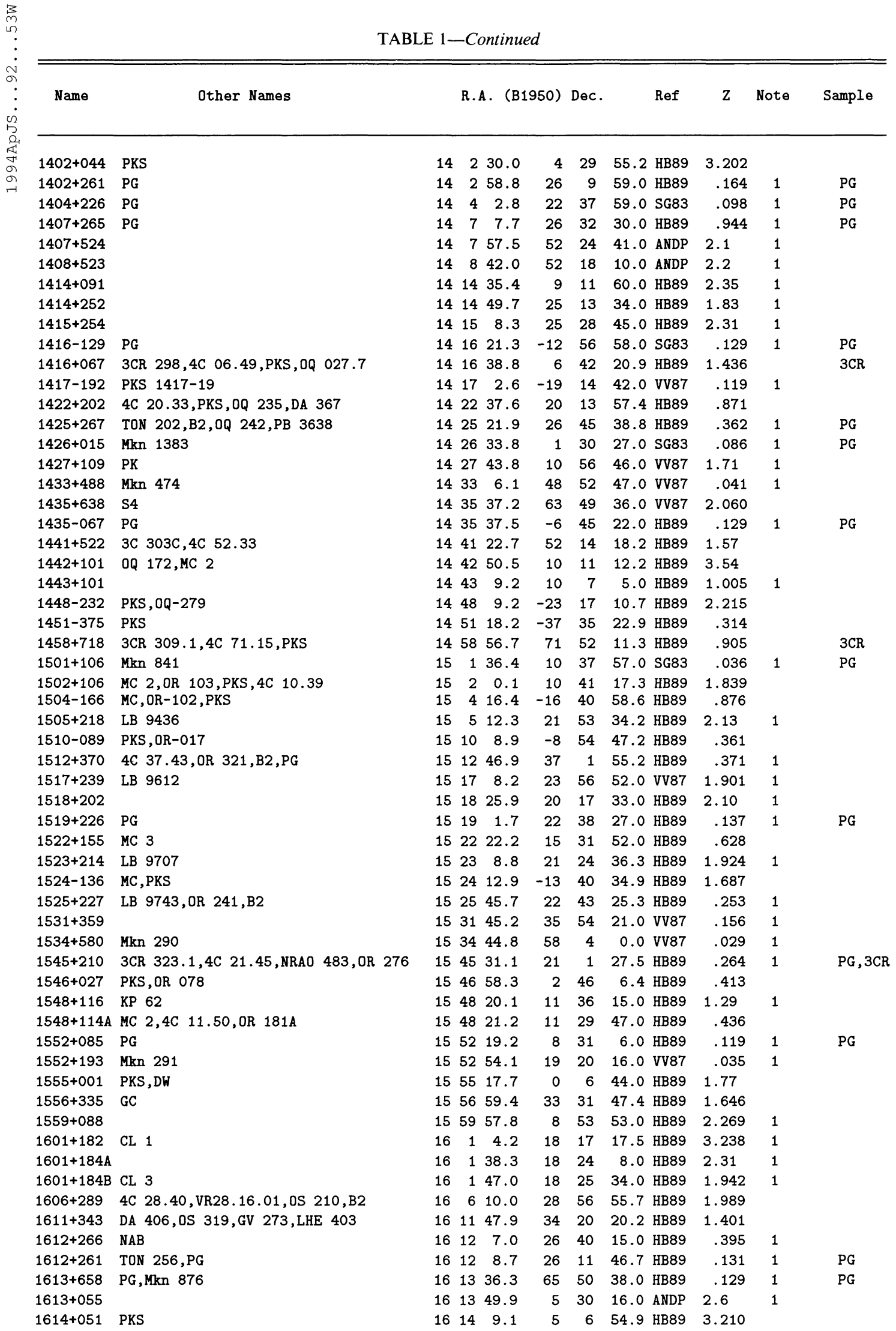




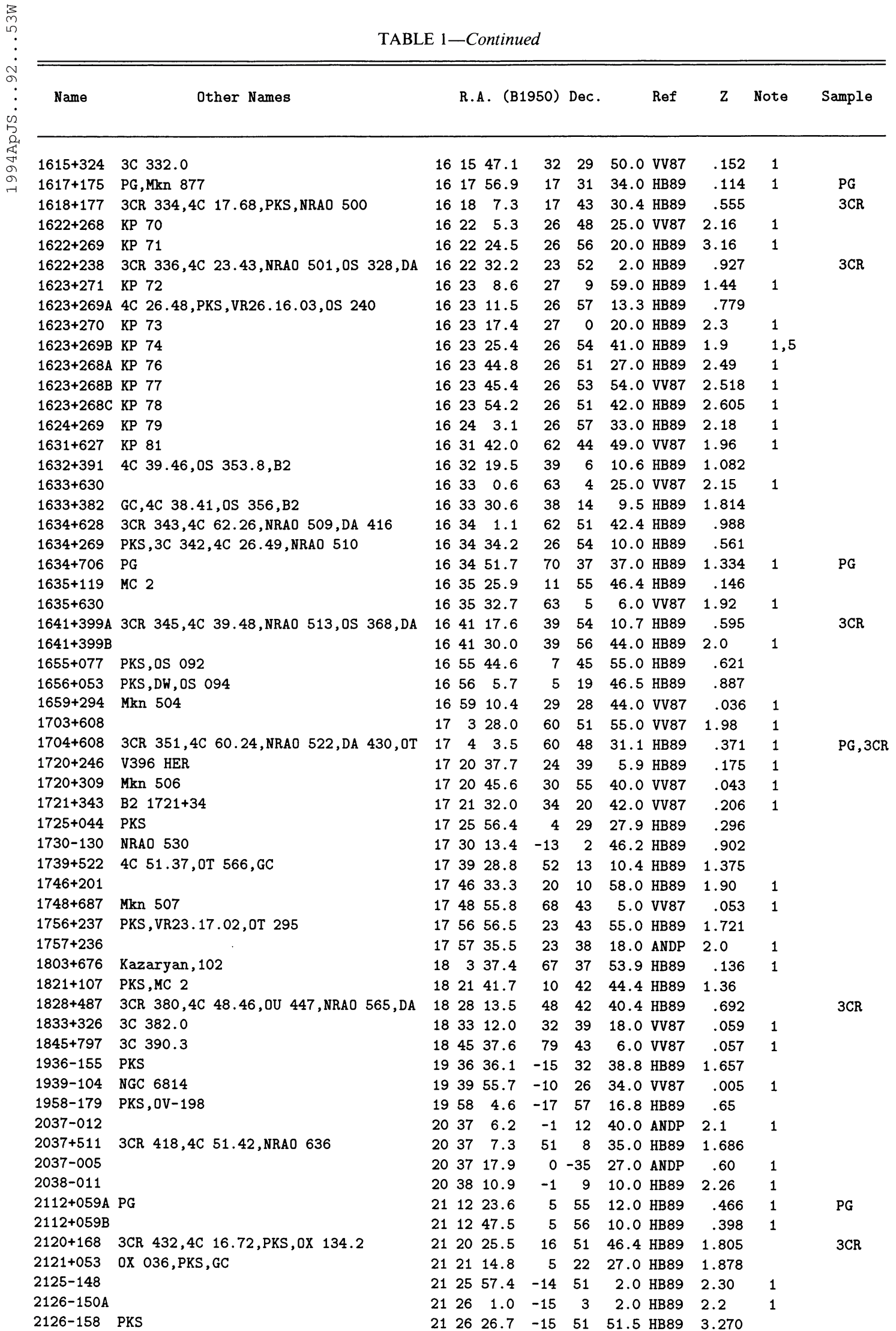


TABLE 1-Continued

\begin{tabular}{|c|c|c|c|c|c|c|c|c|c|c|c|}
\hline Name & Other Names & & R. & A. (B1 & 950) & Dec. & & Ref & $z$ & Note & Sample \\
\hline $2126-150 B$ & & 21 & 26 & 34.1 & -15 & 2 & 39.0 & HB89 & 2.1 & 1 & \\
\hline $2128-123$ & PHL 1598, PKS & 21 & 28 & 52.7 & -12 & 20 & 20.1 & HB89 & .501 & & \\
\hline $2130+099$ & II $\mathrm{ZW} 136, \mathrm{PG}$ & 21 & 30 & 1.3 & 9 & 54 & 59.0 & HB89 & .061 & 1 & PG \\
\hline $2134+004$ & PHL 61, PKS,DA $553,0 \times 057$ & 21 & 34 & 5.3 & 0 & 28 & 25.0 & HB89 & 1.936 & & \\
\hline $2135-147$ & PKS,PHL $1657,0 \mathrm{X}-158$, MSH $21-115$ & 21 & 35 & 1.2 & -14 & 46 & 27.3 & HB89 & .20 & & \\
\hline $2141+175$ & PKS, OX 169, MC 3 & 21 & 41 & 13.8 & 17 & 30 & 2.3 & HB89 & .213 & & \\
\hline $2141+037$ & & 21 & 41 & 40.5 & 3 & 45 & 7.0 & HB89 & 1.8 & 1 & \\
\hline $2143+040$ & & 21 & 43 & 10.8 & 4 & 3 & 59.0 & HB89 & 2.0 & 1 & \\
\hline $2143-156$ & PKS, $0 X-173$ & 21 & 43 & 38.8 & -15 & 39 & 36.7 & HB89 & .701 & & \\
\hline $2145+067$ & PKS,4C $06.69,0 \times 076.1$, DA 562 & 21 & 45 & 36.1 & 6 & 43 & 41.2 & HB89 & .99 & & \\
\hline $2155+034$ & & 21 & 55 & 51.2 & 3 & 29 & 33.0 & HB89 & 1.9 & 1 & \\
\hline $2201+315$ & 4C $31.63, \mathrm{GC}, \mathrm{B} 2$ & 22 & 1 & 1.5 & 31 & 31 & 6.0 & HB89 & .297 & & \\
\hline $2201+171$ & MC 3, PKS, GC & 22 & 1 & 2.9 & 17 & 11 & 19.0 & HB89 & 1.075 & & \\
\hline $2204-408$ & & 22 & 4 & 33.0 & -40 & 51 & 35.0 & HB89 & 3.170 & 1 & \\
\hline $2206-474$ & NGC 7213 & 22 & 6 & 12.0 & -47 & 25 & 0.0 & vV87 & .006 & 1 & \\
\hline $2209+184$ & PG & 22 & 9 & 30.2 & 18 & 27 & 1.0 & SG83 & .070 & 1 & PG \\
\hline $2214+139$ & Mkn 304 & 22 & 14 & 45.2 & 13 & 59 & 27.0 & SG83 & .067 & 1 & PG \\
\hline $2216-038$ & $\mathrm{PKS}, 4 \mathrm{C}-03.79,0 \mathrm{Y}-027, \mathrm{MSH} 22-06$ & 22 & 16 & 16.4 & -3 & 50 & 40.3 & HB89 & .901 & & \\
\hline $2221-023$ & $3 C 445.0$ & 22 & 21 & 14.8 & -2 & 21 & 26.0 & VV87 & .057 & 1 & \\
\hline $2223-052$ & 3C $446,4 \mathrm{C}-05.92, \mathrm{PKS}, 0 \mathrm{Y}-039$ & 22 & 23 & 11.1 & -5 & 12 & 17.4 & HB89 & 1.404 & & \\
\hline $2223+210$ & PKS,DA 580, GC & 22 & 23 & 14.8 & 21 & 2 & 50.0 & HB89 & 1.959 & & \\
\hline $2225-055$ & PHL $5200,4 \mathrm{C}-05.93$ & 22 & 25 & 54.0 & -5 & 34 & 16.6 & HB89 & 1.981 & 1 & \\
\hline $2230+114$ & CTA $102,4 \mathrm{C} 11.69, \mathrm{PKS}, 0 \mathrm{Y} 150$ & 22 & 30 & 7.8 & 11 & 28 & 22.8 & HB89 & 1.037 & & \\
\hline $2232+134$ & & 22 & 32 & 50.5 & 13 & 24 & 35.0 & HB89 & 1.021 & 1 & \\
\hline $2233+134$ & PG & 22 & 33 & 39.8 & 13 & 28 & 21.0 & HB89 & .325 & 1 & PG \\
\hline $2233+136$ & & 22 & 33 & 59.4 & 13 & 41 & 39.0 & HB89 & 3.204 & 1 & \\
\hline $2234+282$ & B2, CTD 135 & 22 & 34 & 1.7 & 28 & 13 & 21.0 & HB89 & .795 & & \\
\hline $2251+158$ & 3CR $454.3,4 \mathrm{C} 15.76, \mathrm{PKS}, \mathrm{OY} 185$ & 22 & 51 & 29.5 & 15 & 52 & 54.6 & HB89 & .859 & & $3 \mathrm{CR}$ \\
\hline $2251+113$ & PKS,4C $11.72,0 Y 186$ & 22 & 51 & 40.6 & 11 & 20 & 39.6 & HB89 & .323 & 1 & PG \\
\hline $2253+417$ & OY $489, \mathrm{GC}$ & 22 & 53 & 19.8 & 41 & 46 & 53.0 & HB89 & 1.476 & & \\
\hline $2254+024$ & PKS, OY 091.3,GC & 22 & 54 & 44.6 & 2 & 27 & 13.8 & HB89 & 2.09 & & \\
\hline $2255+416$ & & 22 & 55 & 5.0 & 41 & 38 & 13.9 & BREG & .942 & 1 & \\
\hline $2300+086$ & NGC 7469 & 23 & 0 & 44.4 & 8 & 36 & 16.0 & vv87 & .017 & 1 & \\
\hline $2301+223$ & Mkn 315 & 23 & 1 & 35.7 & 22 & 21 & 16.0 & vv87 & .040 & 1 & \\
\hline $2305+187$ & 4C $18.68,0 Z 108$, PKS, NRAO 703 & 23 & 5 & 17.1 & 18 & 45 & 5.4 & HB89 & .313 & & \\
\hline $2316-000$ & NGC 7603 & 23 & 16 & 22.9 & 0 & -1 & 47.0 & vv87 & .029 & 1 & \\
\hline $2344+092$ & PKS, 4C $09.74,0 Z$ 073.5, PB 5532 & 23 & 44 & 3.8 & 9 & 14 & 5.5 & HB89 & .672 & 1 & PG \\
\hline $2345-167$ & PKS , 0Z-176, MC & 23 & 45 & 27.7 & -16 & 47 & 52.9 & HB89 & .576 & & \\
\hline $2345+184$ & $3 \mathrm{C} 467$ & 23 & 45 & 57.0 & 18 & 27 & 29.0 & vv87 & .631 & 1 & \\
\hline $2348-014$ & WEE 180 & 23 & 48 & 54.3 & -1 & 27 & 42.0 & HB89 & 2.04 & 1 & \\
\hline $2349-014$ & PG,PKS, PB 5564 & 23 & 49 & 22.3 & -1 & 25 & 54.0 & HB89 & .174 & 1 & \\
\hline 2349-015 & WEE 183 & 23 & 49 & 42.9 & -1 & 30 & 0.0 & HB89 & 2.20 & 1 & \\
\hline $2353+072 A$ & Mkn 541 & 23 & 53 & 28.4 & 7 & 14 & 41.0 & VV87 & .040 & 1 & \\
\hline $2353+072 B$ & & 23 & 53 & 59.0 & 7 & 14 & 7.0 & VV87 & .342 & 1 & \\
\hline $2357-348$ & & 23 & 57 & 6.0 & -34 & 52 & 0.0 & VV87 & 2.070 & 1 & \\
\hline
\end{tabular}

${ }^{a}$ This table also is published in computer-readable form in the AAS CD-ROM Series, Vol. 2.

NOTES.-(1) Object included in optically selected sample for further analysis. (2) $z$ from Marshall et al. 1984 and differs slightly from HB89. (3) Included in optically selected sample due to independent selection in prism survey (not flagged as "O" or "C" in HB89). (4) Not included in PG "X-ray Sample" since it falls in declination zone generally not observed. (5) Redshift and optical magnitude (see Table 6) from Sramek \& Weedman 1980 and differs from HB89 due to transcription error passed through intermediate references.

REFERENCES.-ANDP, Anderson 1990; BREG, Bregman et al. 1985 and references therein; B2, Colla et al. 1970; GSL86, Green, Schmidt, Liebert 1986; HB89, Hewitt \& Burbidge 1989 (information extracted from computer tapes and includes all data from Hewitt \& Burbidge 1987); SG83, Schmidt \& Green 1983; V87, Véron-Cetty \& Véron 1987 (information extracted from computer tapes and includes all data in ESO Sci. Rept. No. 5). 


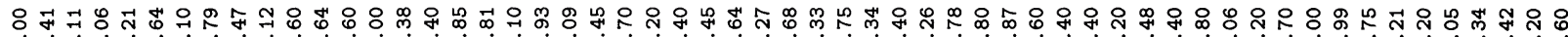
ம் 我 ○ न

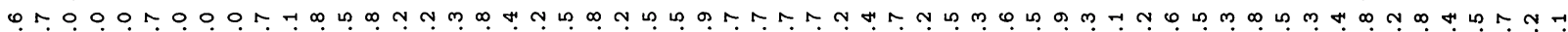

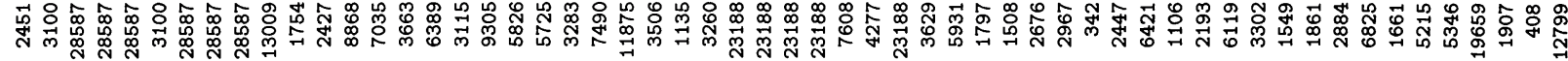

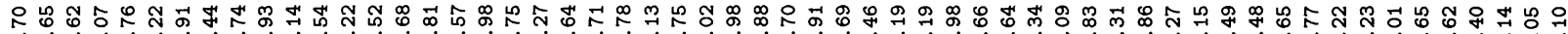
ம்

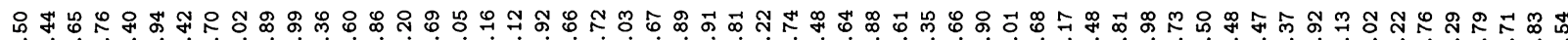
ฟ

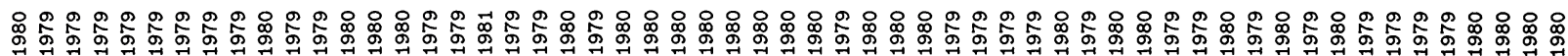

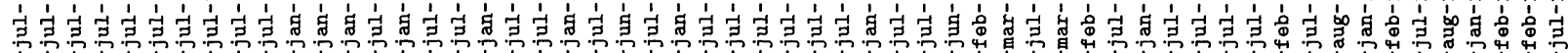

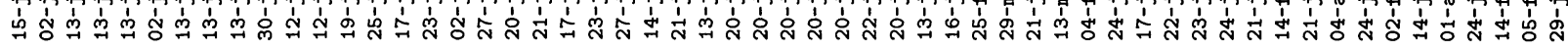

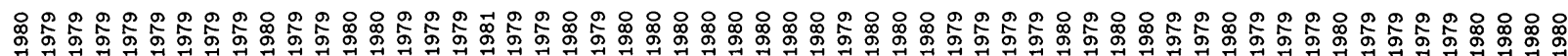

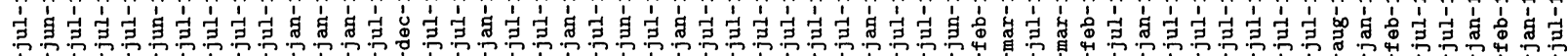

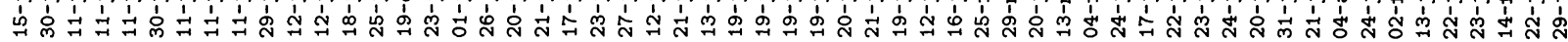

$$
\text { 人 }
$$

$\stackrel{2}{\exists}$

$\stackrel{4}{i}$

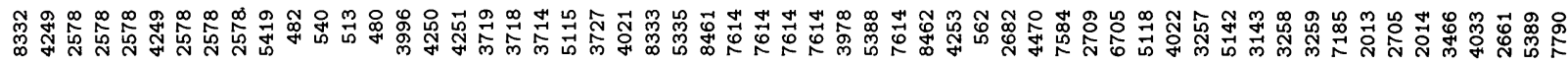

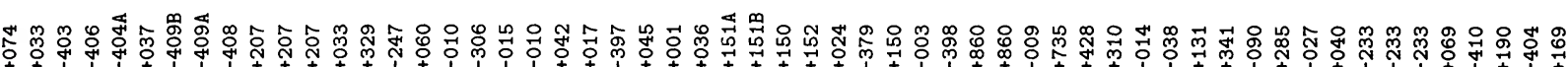

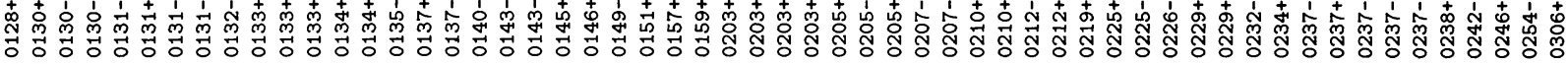

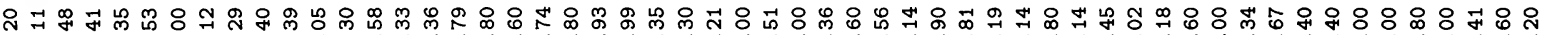
ம

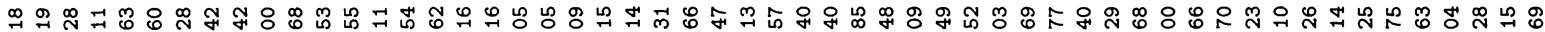
H

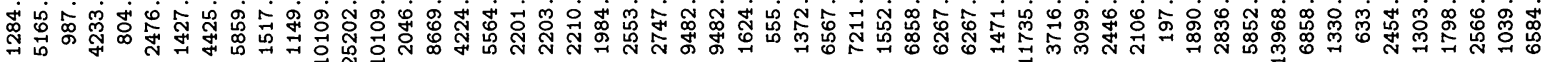

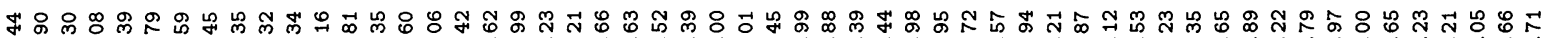

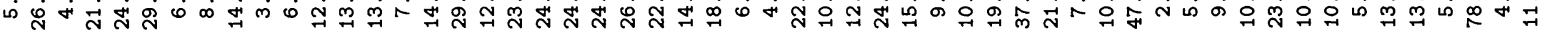

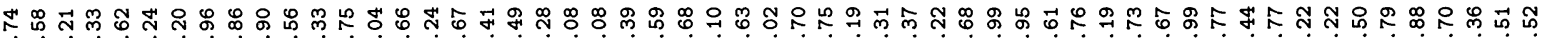

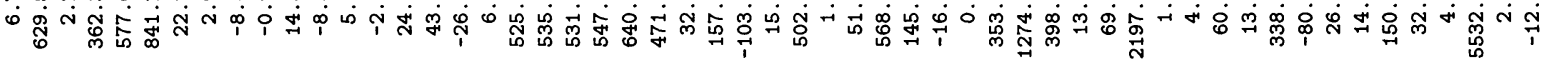

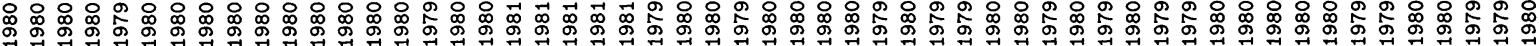

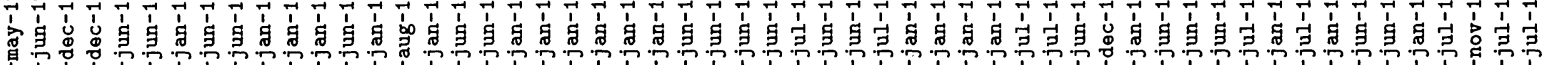

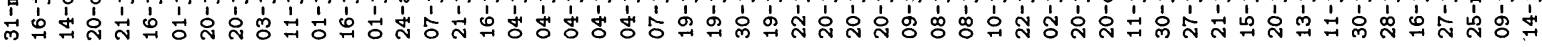

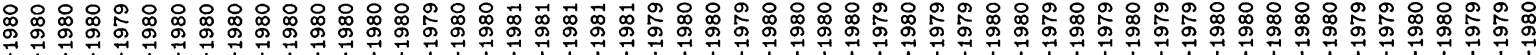

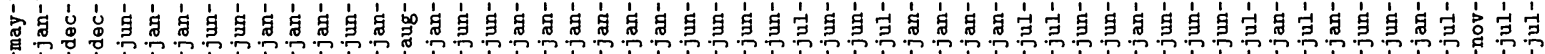

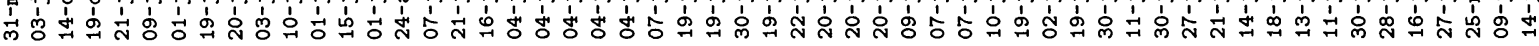

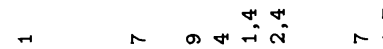

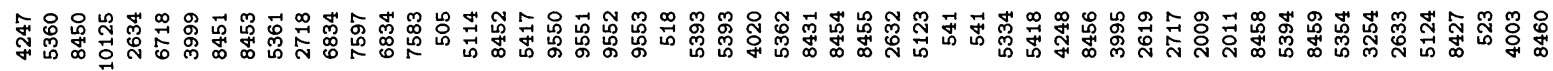

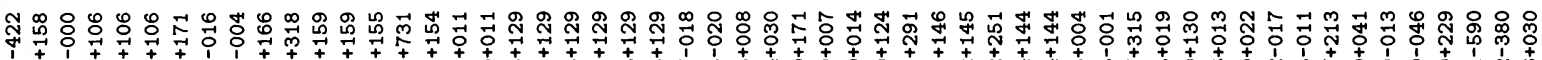

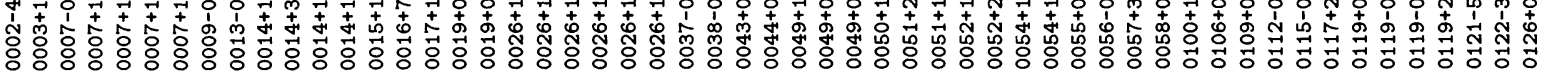




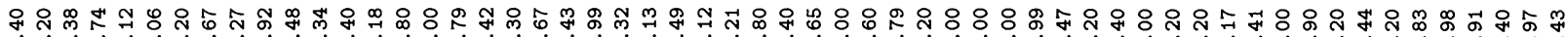

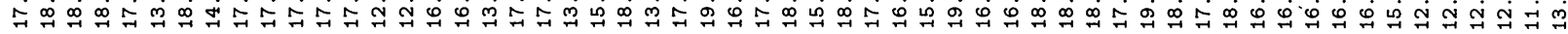

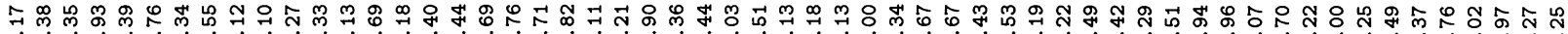

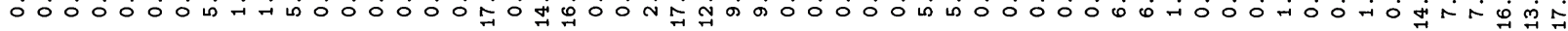
个. ᄀ

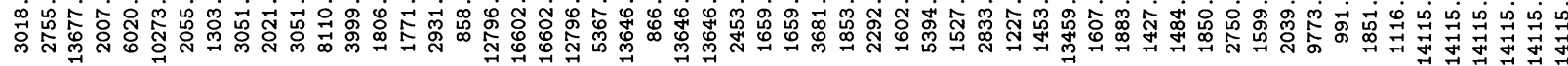

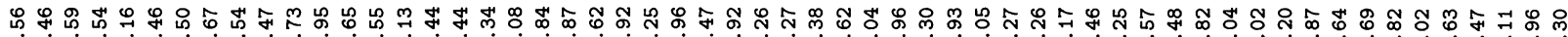

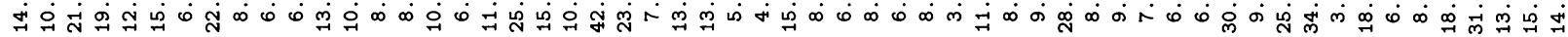

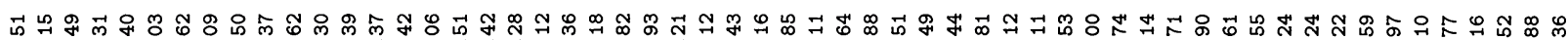

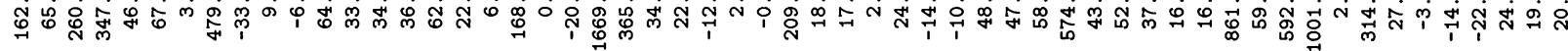

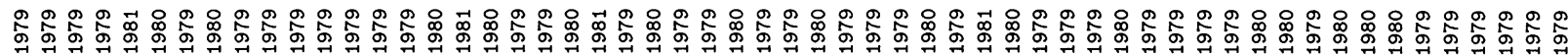

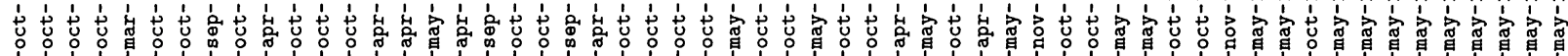

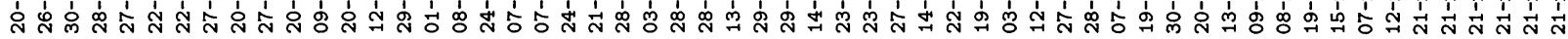

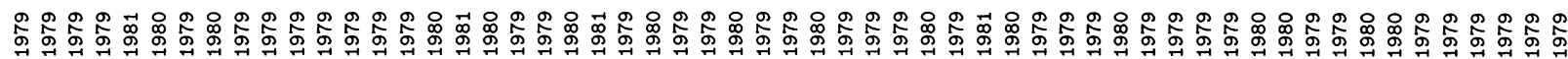

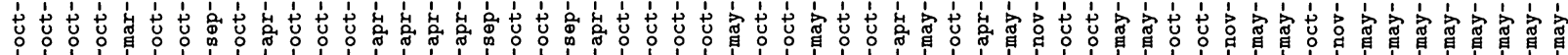

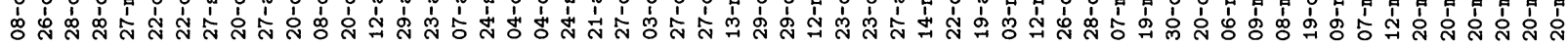
h h

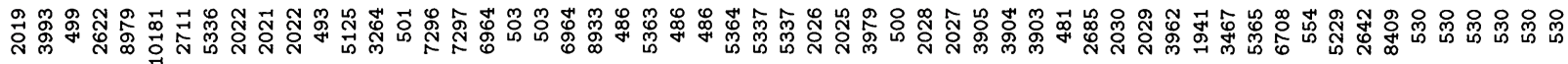

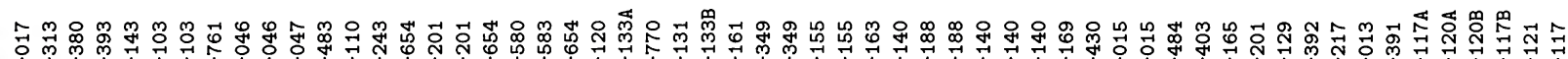

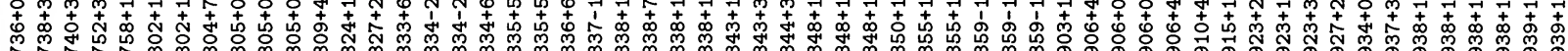

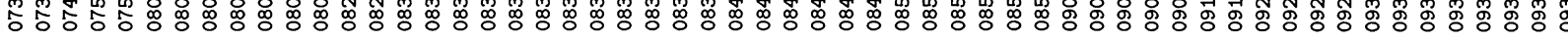

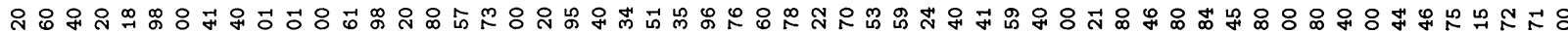
궈

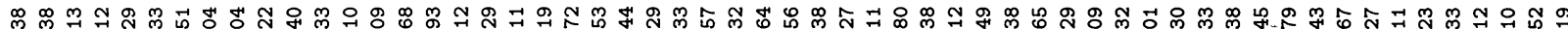

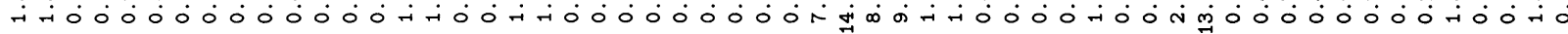
の 0 a 范

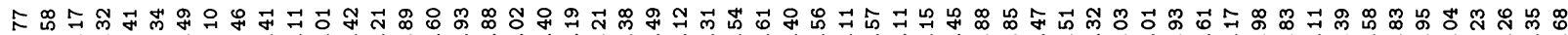

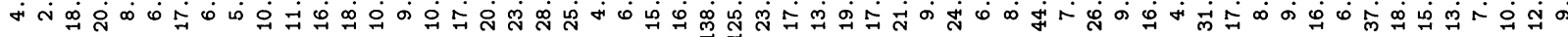

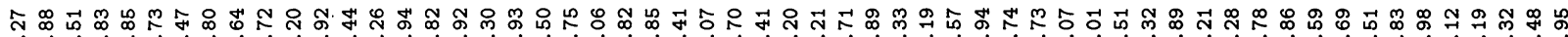

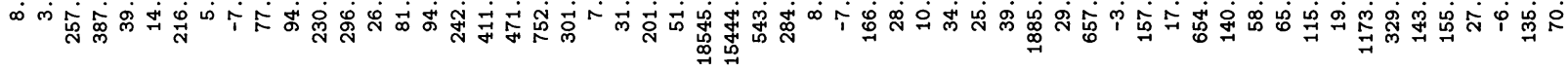

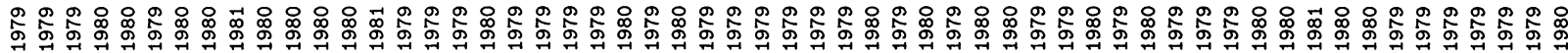

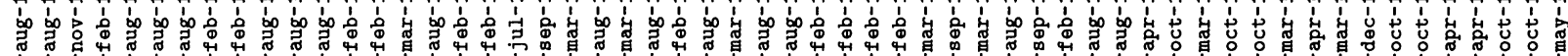

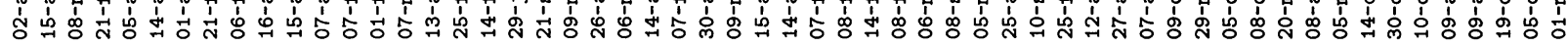

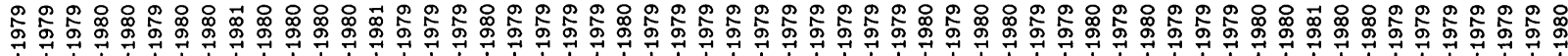
bo

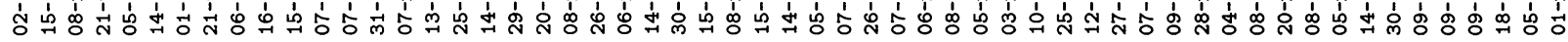

$\stackrel{0}{\circ}$

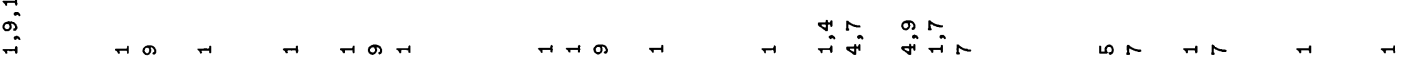

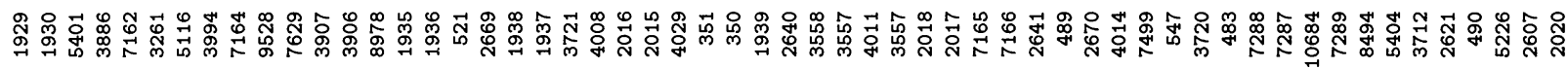

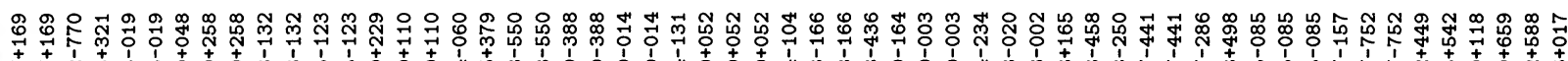

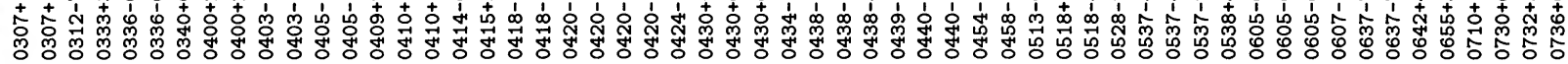




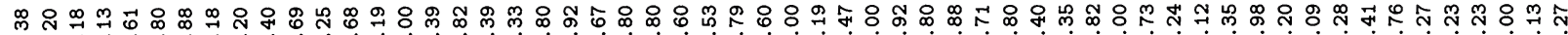
ம்

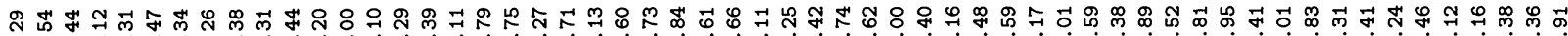

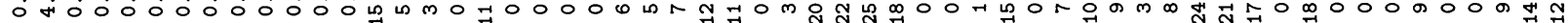

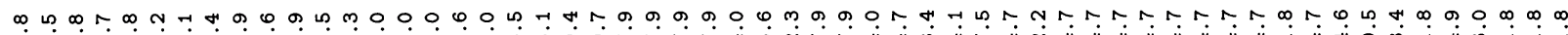

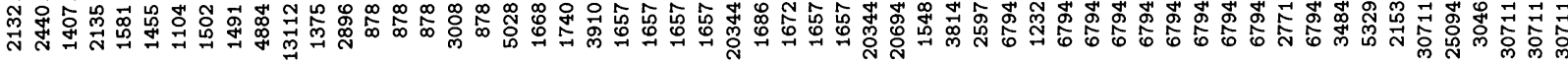

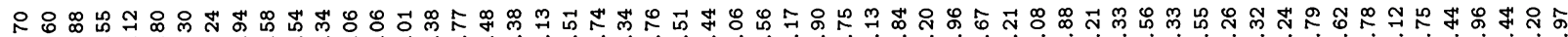

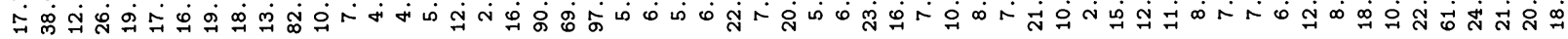

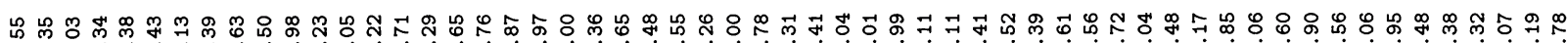

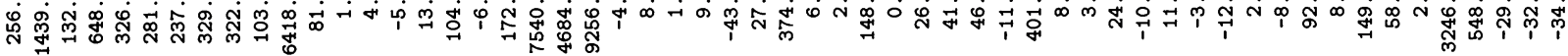

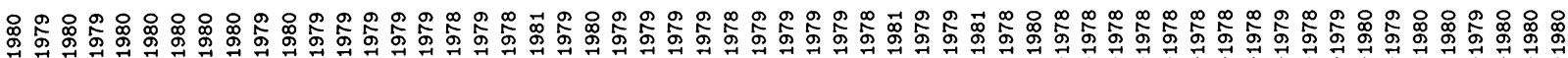

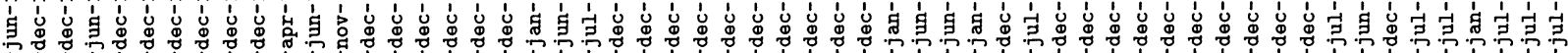

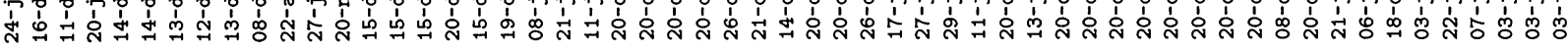

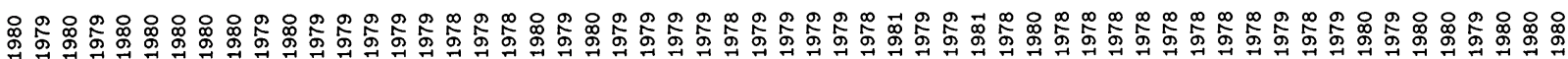

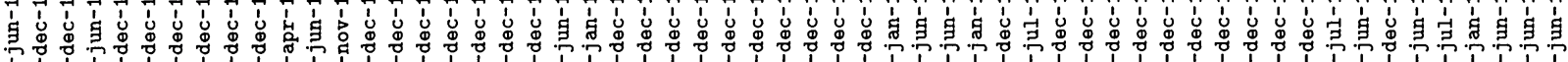

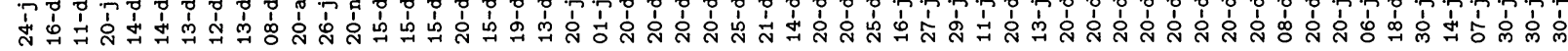

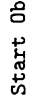

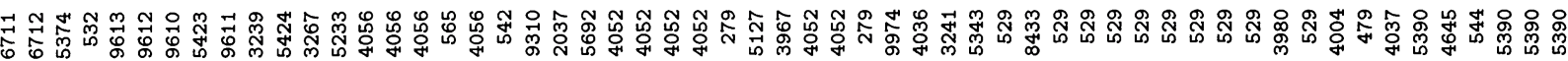

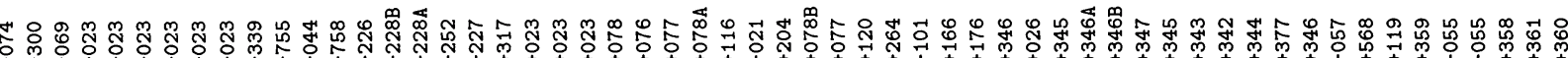

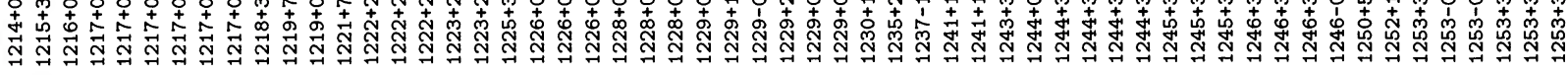

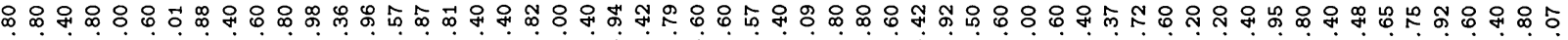
ت

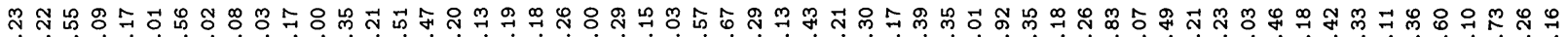
-

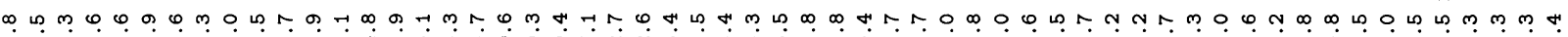

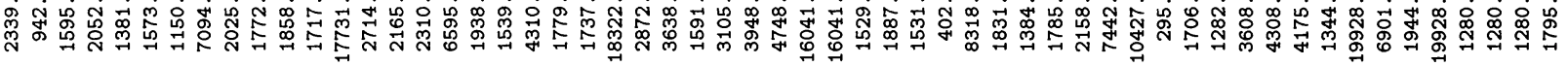

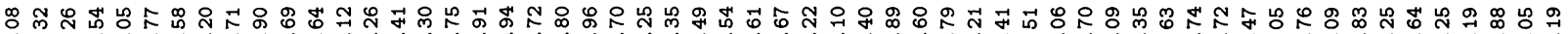
잉 ம

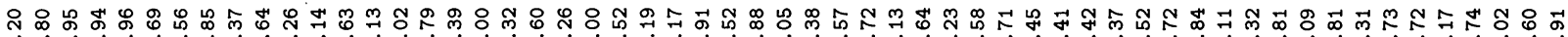

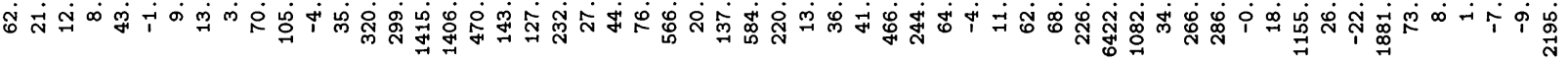

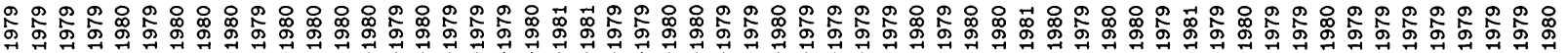

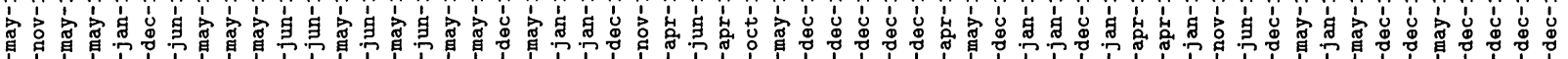

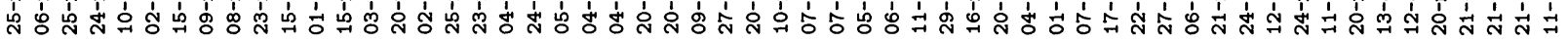

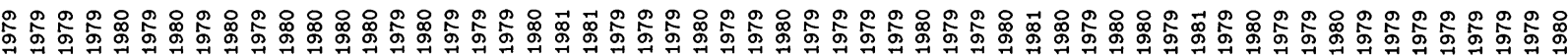

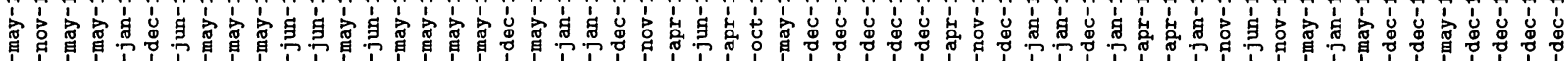

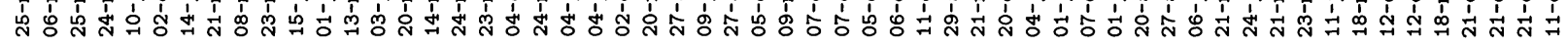

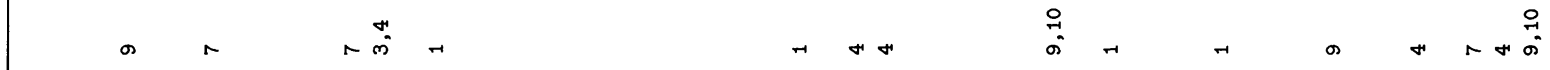

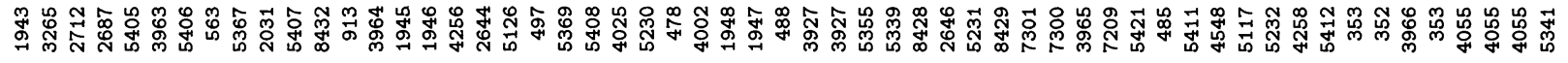

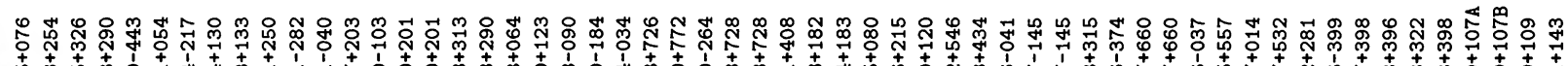

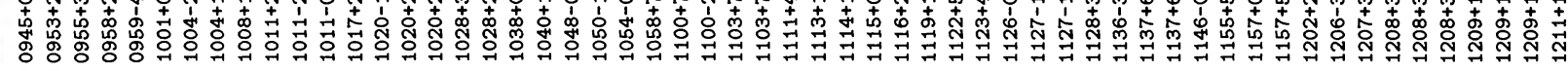




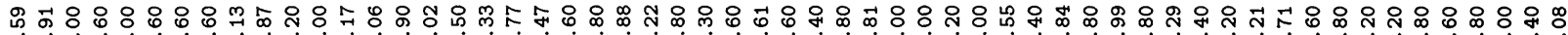

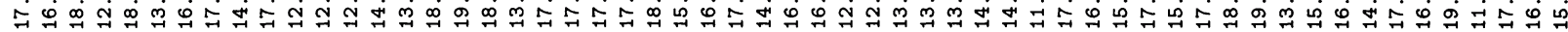

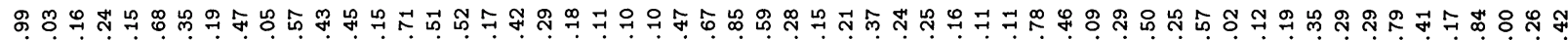

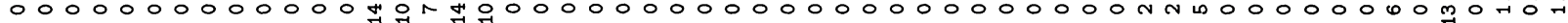

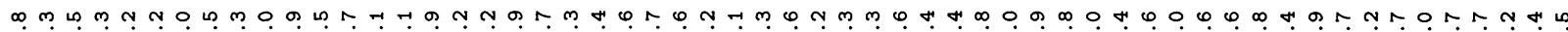

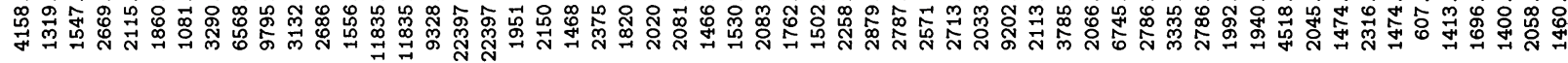

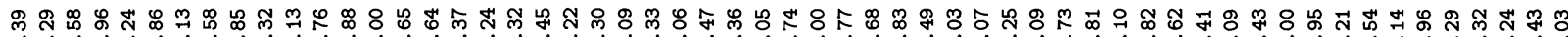
कம

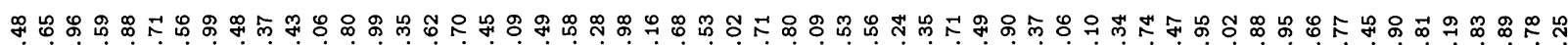

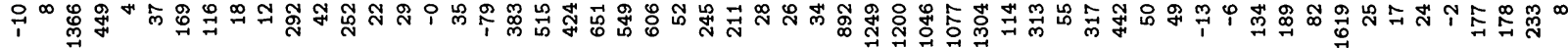

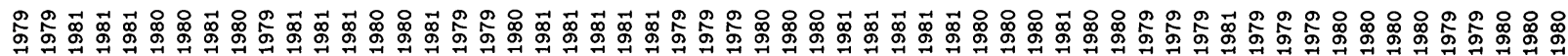

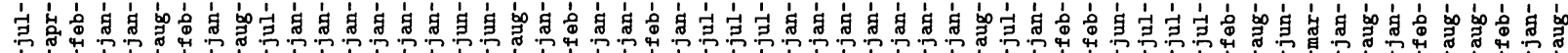

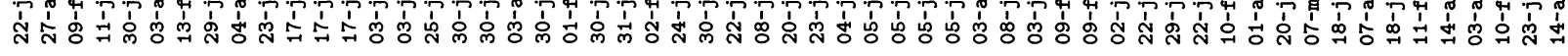

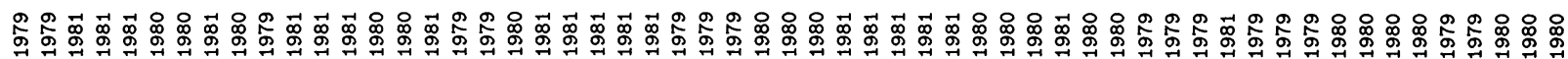

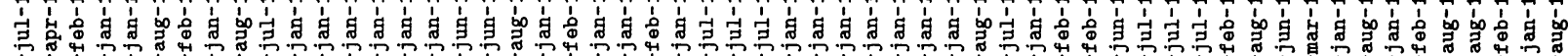

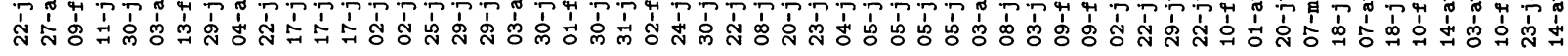

ن)

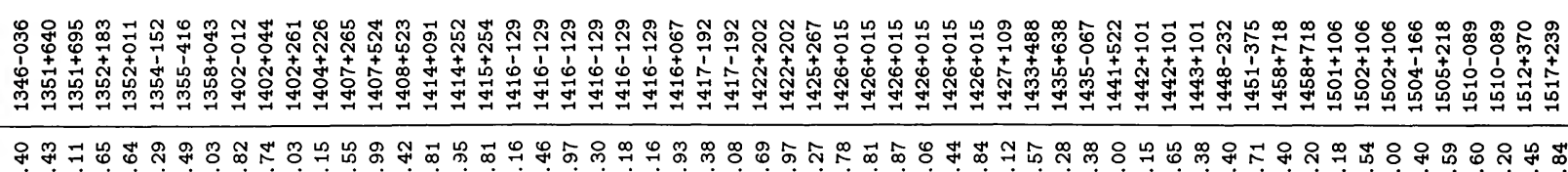

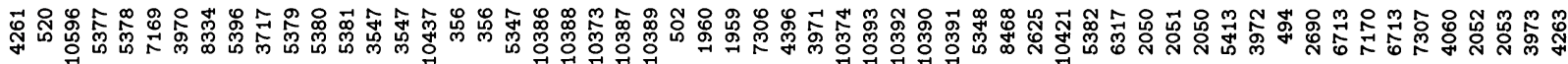

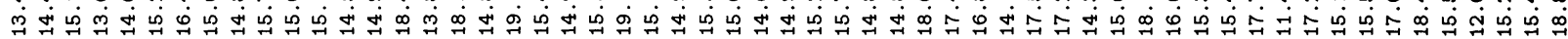

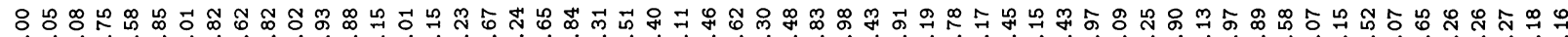
○ல்

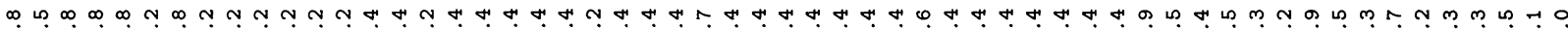

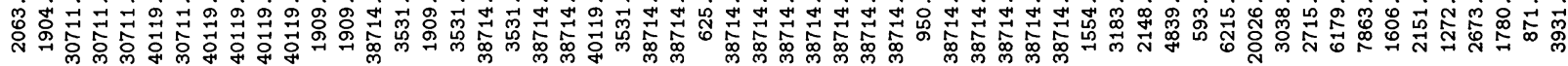

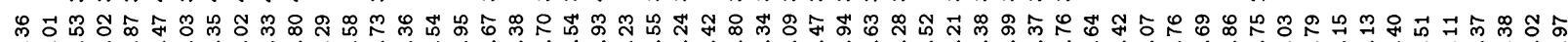

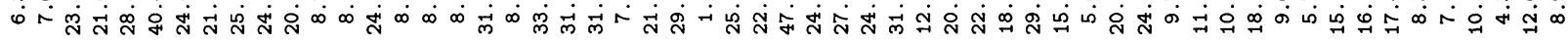

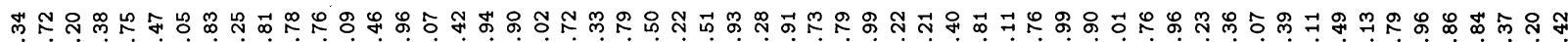

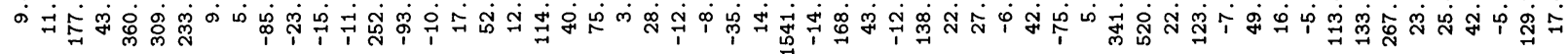

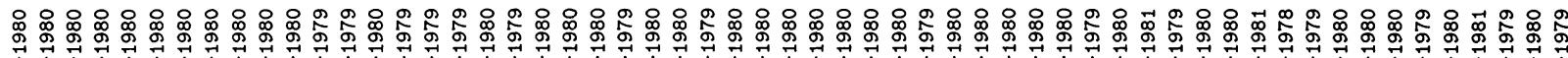

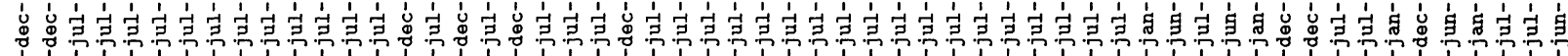

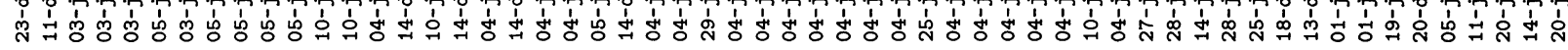

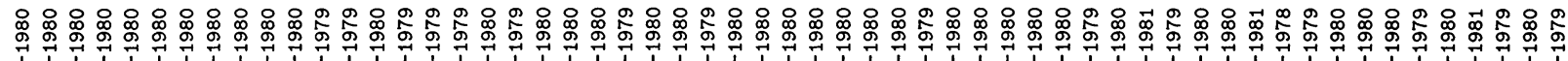

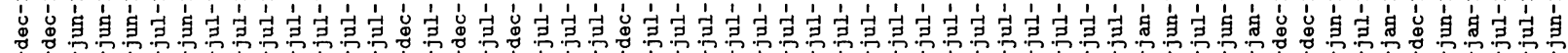

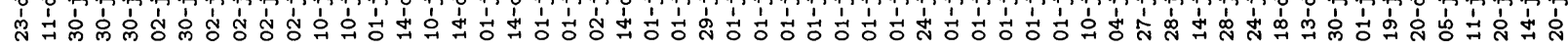

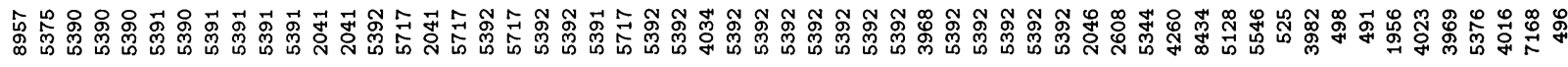

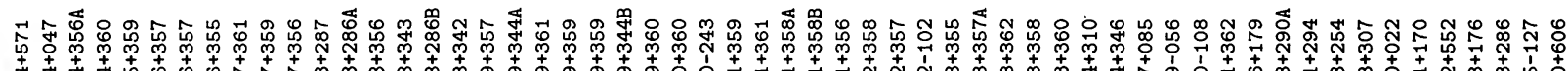

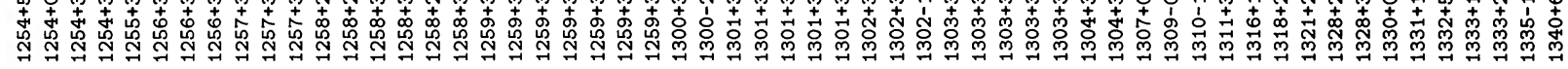




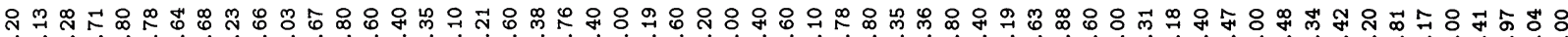
m

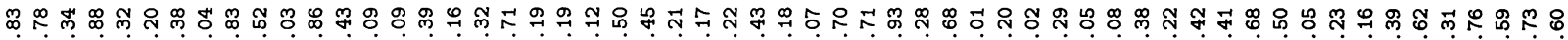
ఐ क人

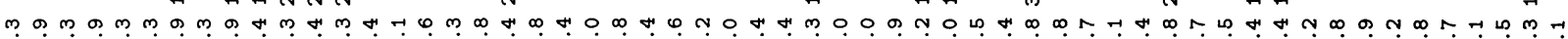

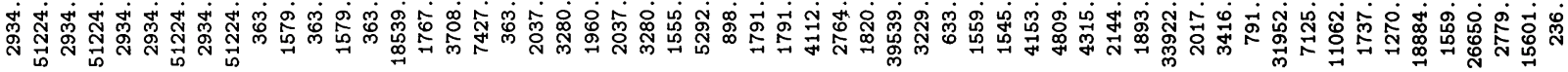

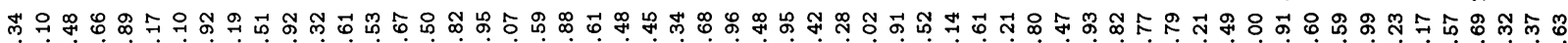
স

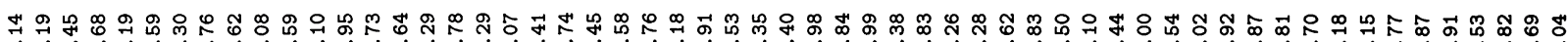

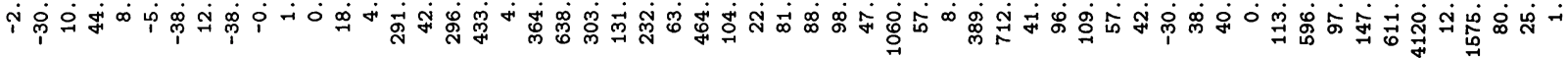

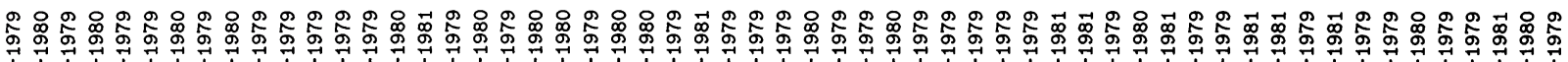

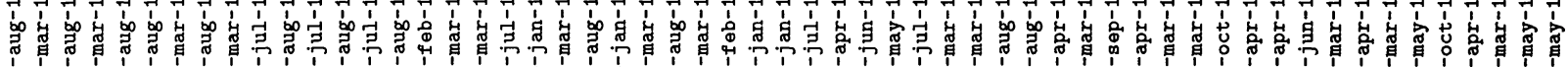

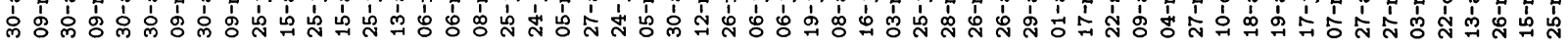

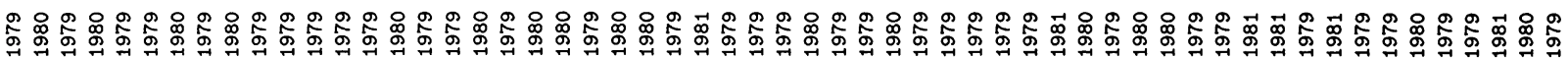

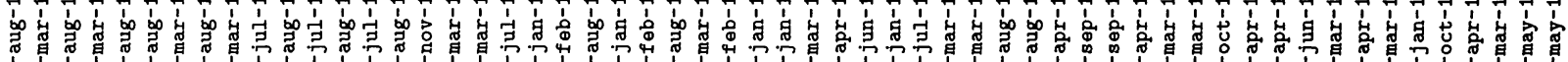

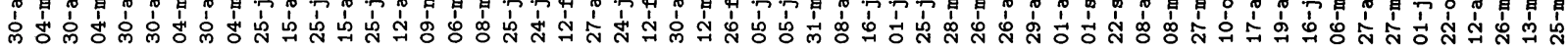

i

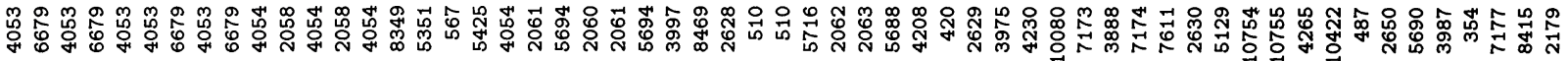

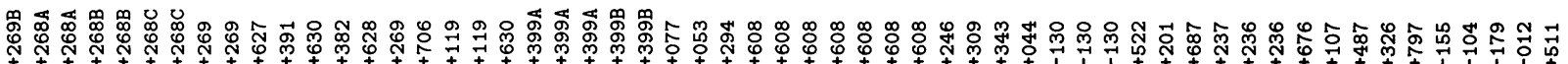

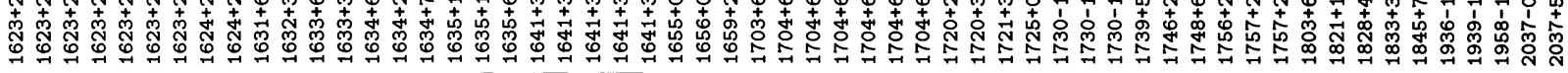

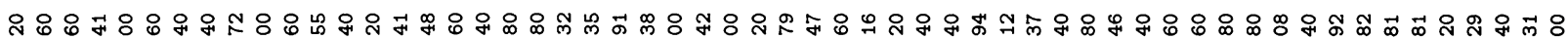

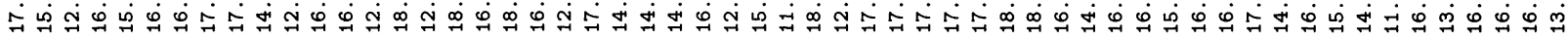

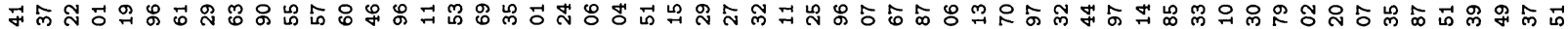

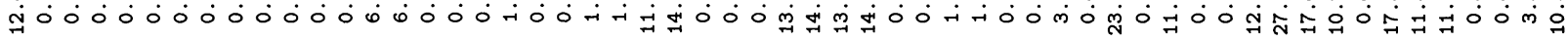

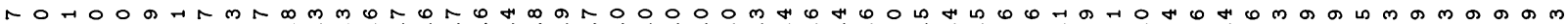

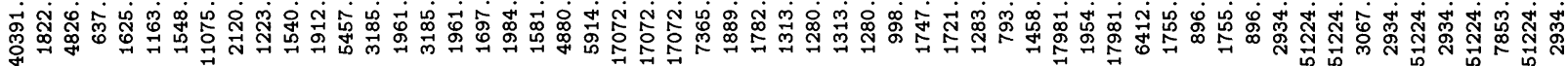

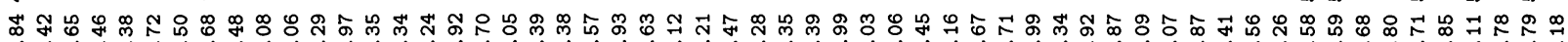

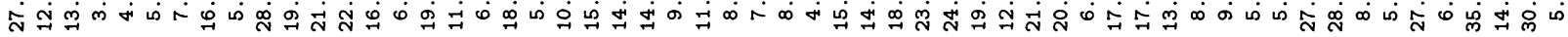

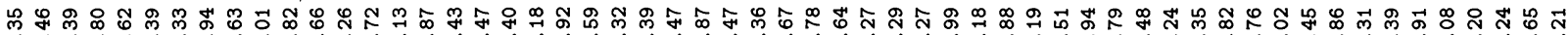

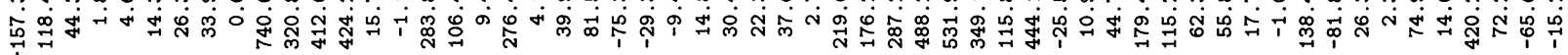

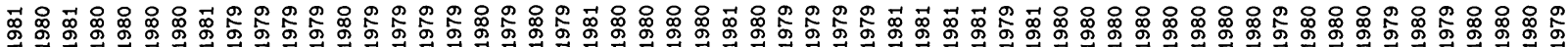

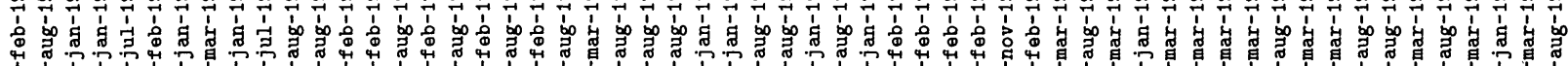

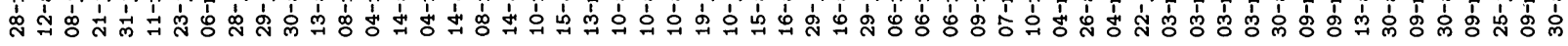

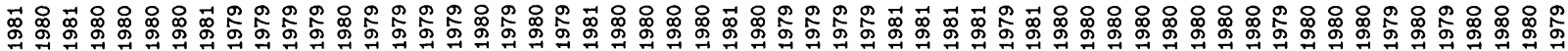

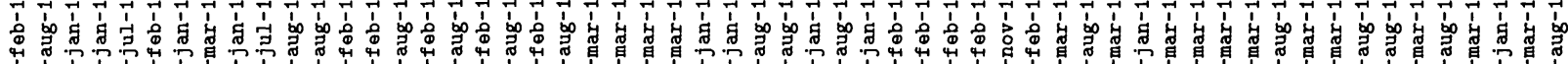

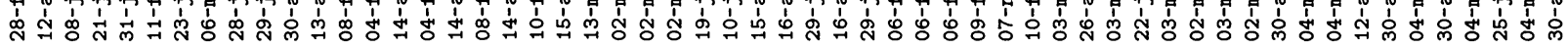

\& Hata H

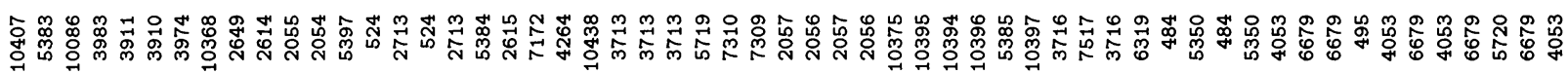

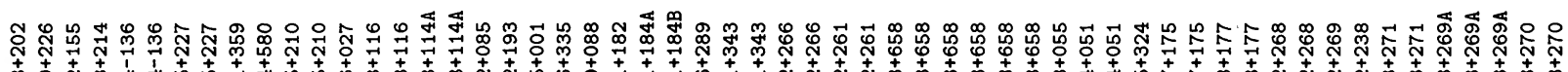

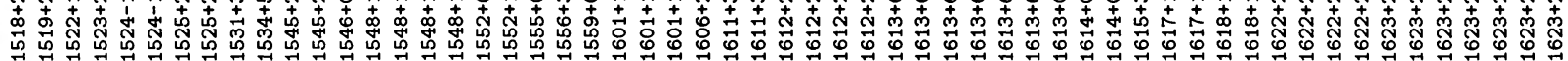




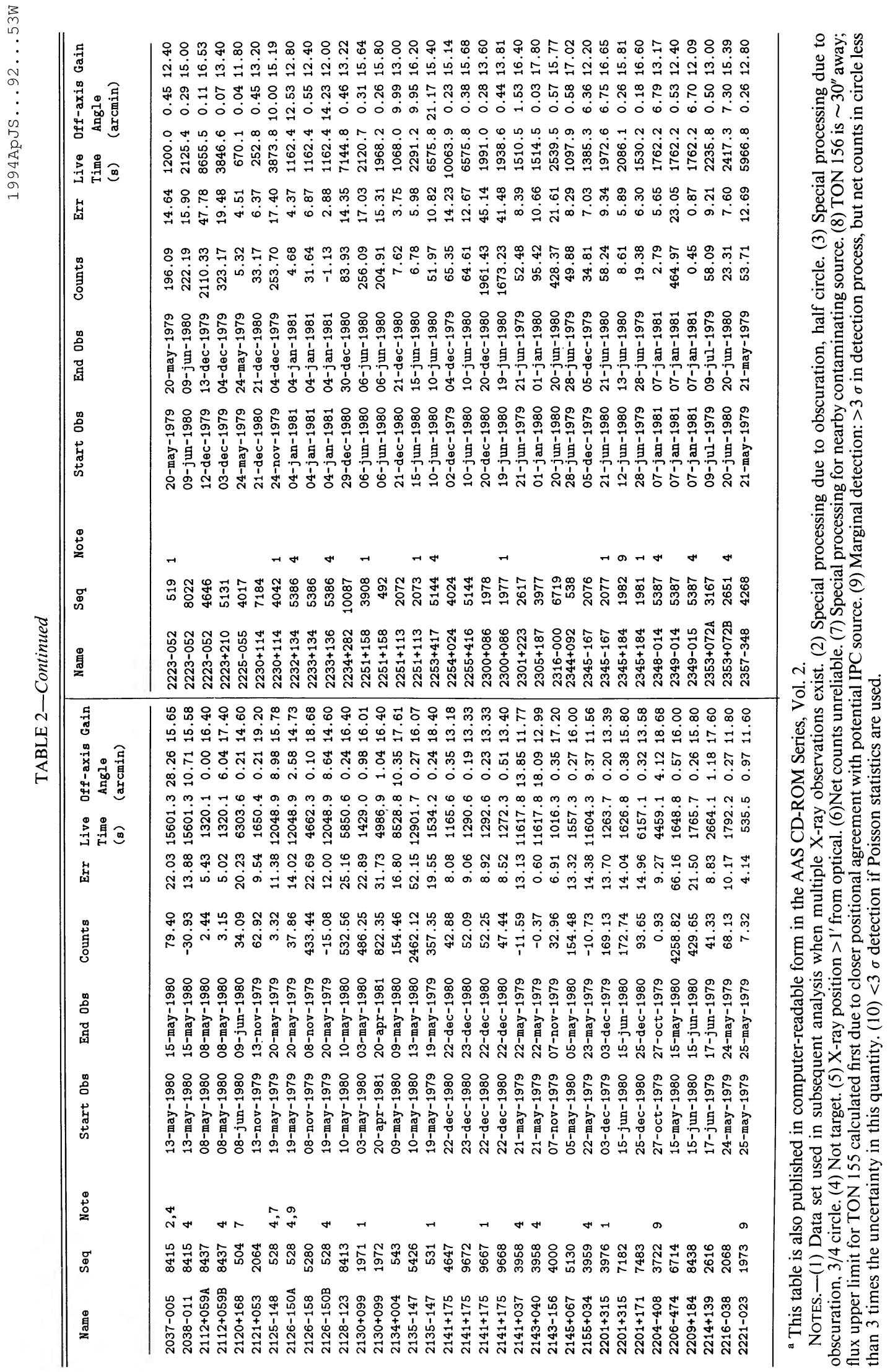

69 
low); these 10 instances are indicated as unreliable with a 6 in the Note column of Table 2.

After this work was essentially complete a problem was found in the IPC software used to convert counts from pulseheight (PH) to PI bins. This error enters through a roll-angle calculation, so sources near the telescope/detector axis are affected only slightly. For the sources more than a few arcminutes off-axis, we determined the effect of this error on our results by making a comparison of a random subset of quasars with and without the error corrected. We found that for $\sim \frac{2}{3}$ of the objects, an additional uncertainty of $\leq 4 \%$ is introduced by the use of inaccurate local gain factors in the conversion from broad-band PH to broad-band PI counts. For the remaining one-third, the additional uncertainty is $\sim 4 \%-7 \%$ (although in the case of one marginal detection, we found a change of $\sim 30 \%$ ). In all cases, the changes were less than $0.8 \sigma$, and this additional uncertainty has only a negligible effect on any of our subsequent analyses. Similarly, we would also expect a few of the objects around our detection threshold to change their detection status.

For estimating the fluxes, we corrected the net source counts for counts lost due to mirror scattering, the telescope/IPC point-spread function (PSF), vignetting, and detector dead time (generally $\sim 4 \%$ ). The effects of mirror scattering and energy-dependent vignetting were removed using the effectivearea table appropriate for a $3^{\prime}$ radius circle as a function of its off-axis distance (Harnden et al. 1984). The fraction of counts lost due to the PSF is a strong function of their pulse height and thus differs among sources. In most cases this correction was made to the counts in each individual pulse height $(\mathrm{PH})$ channel before converting to pulse-height invariant (PI) bins and combining them to compute the flux. The percentage correction computed in this way is strongly peaked at $1.8 \%$. Tables $3 \mathrm{~A}, 3 \mathrm{~B}$, and $3 \mathrm{C}$ list the broad-band and monochromatic X-ray fluxes or $3 \sigma$ upper limits (in the observer's energy frame), derived from the quoted count rates corrected as described above. Fluxes were computed assuming Galactic absorption, and a power-law energy distribution $\left(F_{\nu} \propto \nu^{-\alpha_{x}}\right)$ for $\alpha_{x}=0.0$, $0.5,1.0,1.5,2.0$, respectively. This set covers the range of slopes typically found in the IPC energy band for quasars (Wilkes \& Elvis 1987).

Galactic $N_{\mathrm{H}}$ values were determined by interpolation from the Bell Laboratories survey (Stark et al. 1992) and were set for each IPC field based upon the coordinates of the center of the IPC field. ${ }^{7}$ For southern quasar positions, which are not covered by this survey (typically $\delta<-42^{\circ}$ ), the maps of Heiles \& Cleary (1979) were used, as noted with a 1 in the Note column of Table 3A.

Statistical errors on the fluxes and luminosities can be derived from the fractional error in the count rate (Table 2). Errors due to the uncertainty in the spectral slope can be estimated from the range of flux present in Tables 3A-3C. For $\mathrm{X}$-ray nondetections the $3 \sigma$ upper limits on fluxes and luminosities given in Tables $3 \mathrm{~A}-3 \mathrm{C}$ are derived conservatively from the data in Table 2 , using [ $\max (0$, counts $)+3 *$ Err] as the count rate. We have used a Friedmann cosmology with

\footnotetext{
${ }^{7}$ N.B. Thus all objects in a given field will use the same $N_{\mathrm{H}}$, but two different observations of the same object may have slightly different $N_{\mathrm{H}}$ values. The difference is always well within the uncertainties.
}

Hubble constant of $H_{0}=50 \mathrm{~km} \mathrm{~s}^{-1} \mathrm{Mpc}^{-1}$ and deceleration parameter of $q_{0}=0.0$ to compute luminosities. In Tables 3A3C, X-ray luminosities for the $0.2-4.5 \mathrm{keV}$ band and $2 \mathrm{keV}$ monochromatic are referenced to the source energy frame.

\subsection{Quasar Positions Close to a Nearby, Contaminating Source}

Sample objects with X-ray sources detected less than 6' away were individually inspected to determine the significance of contamination introduced by the nearby source. If contamination was found to be insignificant ( $<5 \%$ of the total counts from the nearby source falling within a 3 ' radius circle centered the quasar position), as was usually the case for separations greater than 4 ', the processing was resumed as normal. Objects suffering significant contamination from a single nearby source were processed as described below. Those with significant contamination from more than one nearby source were not processed and are listed in Table 5 (see below).

For objects contaminated by a single source, a circle was centered on the target and another circle with a $3^{\prime}$ radius was centered on the contaminating source. Counts were determined for the target and contaminating source $3^{\prime}$ circles excluding the region of geometric overlap $\left(C_{1}, C_{2}\right)$, and counts were also determined for the overlap region $\left(C_{3}\right.$; see Fig. 1). Background counts were then determined for identical regions of the background map, $B_{1}, B_{2}, B_{3}$, and net broad-band counts in the three regions $N_{1}, N_{2}, N_{3}$. The fraction of source counts in the overlap region is primarily a function of the point response and therefore depends on geometry and photon energy. Using a maximum-likelihood formulation assuming Poisson distributions for each region and treating the fraction of the source counts in the overlap region as a parameter to be fitted allows us to make preliminary estimates of the source counts $S_{1}, S_{2}$ from the target object and contaminating source, respectively:

$$
\begin{aligned}
& S_{1}=N_{1}+\frac{N_{1} N_{3}}{N_{1}+N_{2}} \\
& S_{2}=N_{2}+\frac{N_{2} N_{3}}{N_{1}+N_{2}} .
\end{aligned}
$$

The error $\sigma_{1}$, representing $68 \%$ confidence with the formulation above, for the target source is given by

$$
\begin{aligned}
& \sigma_{1}= \\
& \frac{\sqrt{C_{1}\left[\left(N_{1}+N_{2}\right)^{2}+N_{2} N_{3}\right]^{2}+C_{3} N_{1}^{2}\left(N_{1}+N_{2}\right)^{2}+C_{2} N_{1}^{2} N_{3}^{2}}}{\left(N_{1}+N_{2}\right)^{2}},
\end{aligned}
$$

where we have assumed that the uncertainty in the background counts $\left(B_{1}, B_{2}, B_{3}\right)$ is negligible. The maximum-likelihood calculation was used to determine source existence $\left(S_{1} \geq 3 \sigma_{1}\right)$ as well as to estimate source intensity.

The estimate for quasar counts does not take into account the $\sim 1.8 \%$ of the counts falling outside a $3^{\prime}$ circle, some of which will fall in the neighboring source circle and thereby 


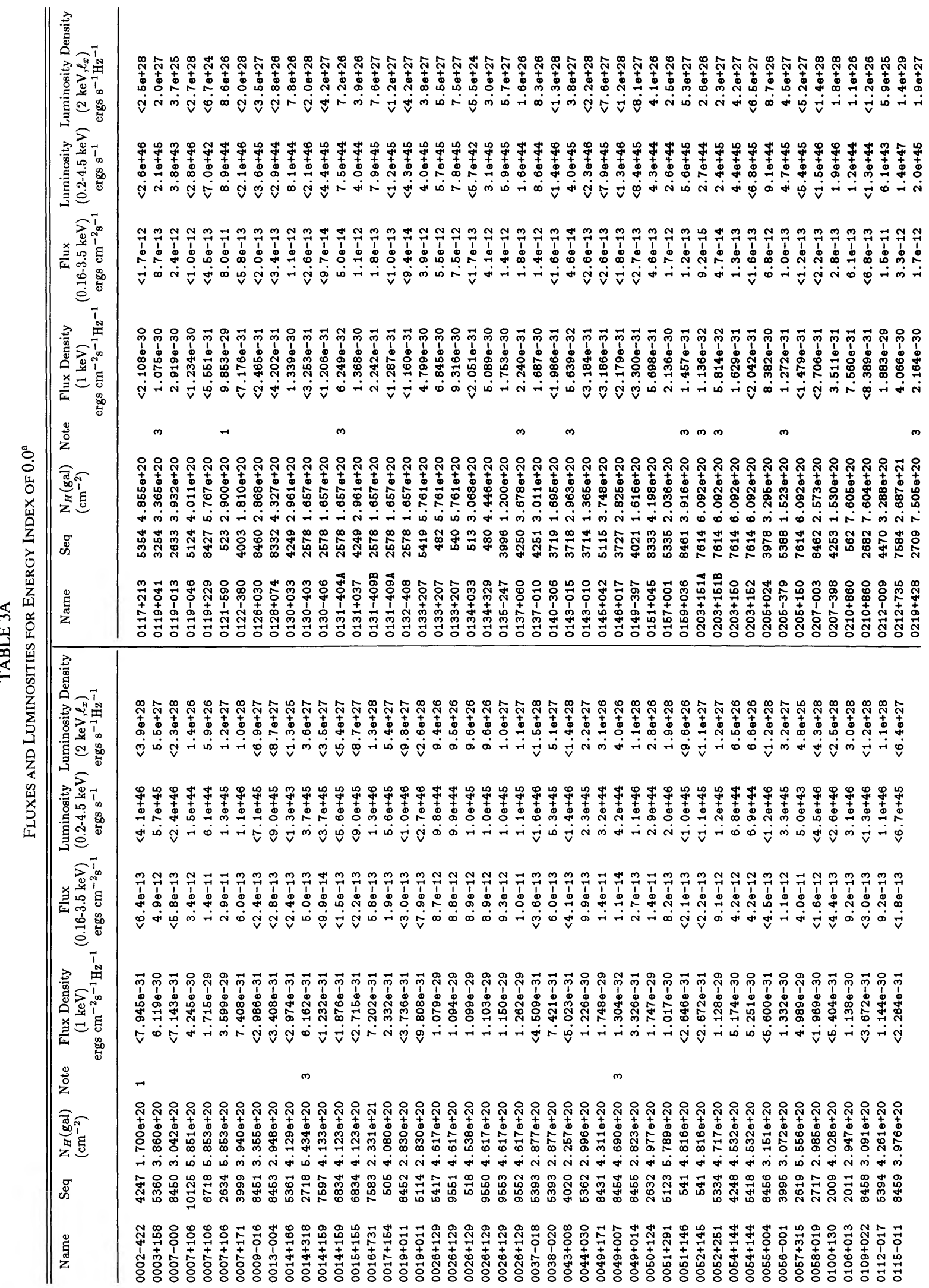


ลงกิง

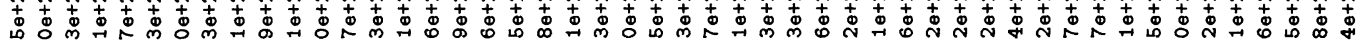

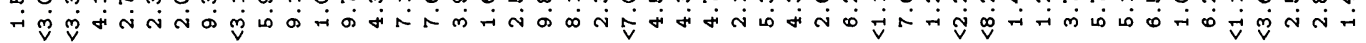

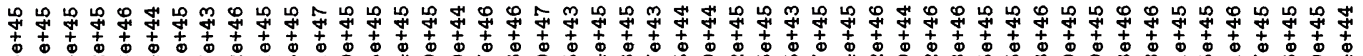

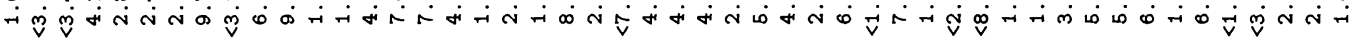

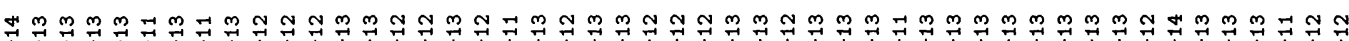

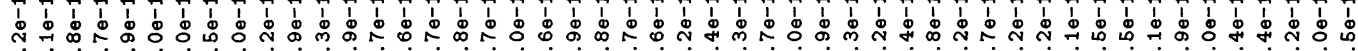

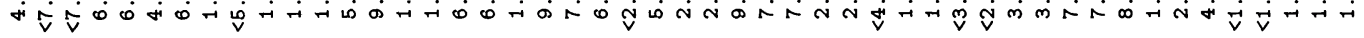

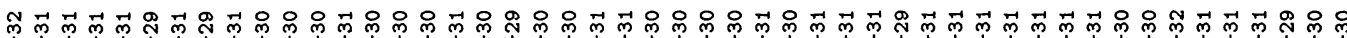

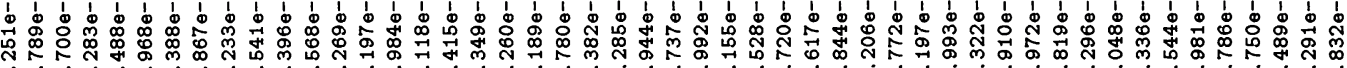
ம

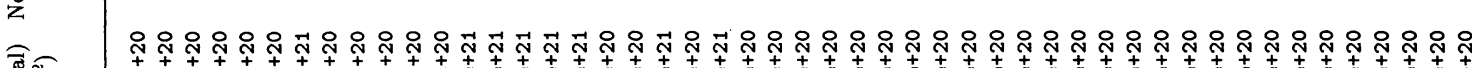

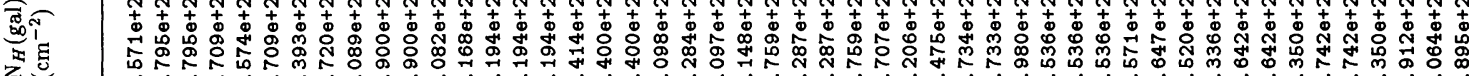

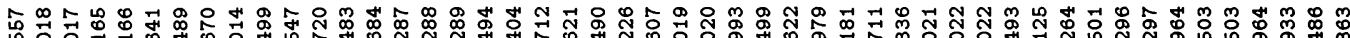

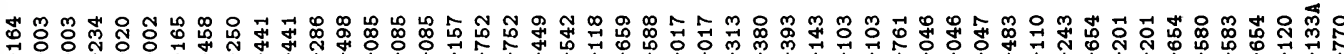

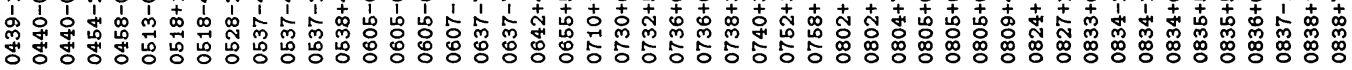

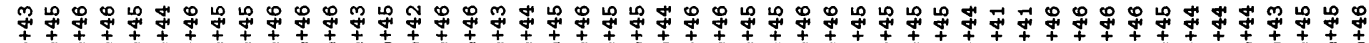

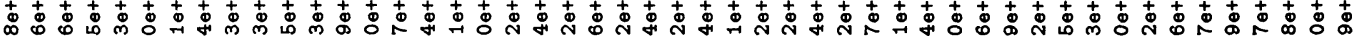

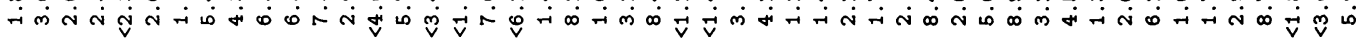

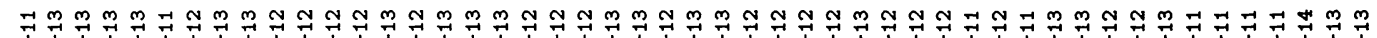

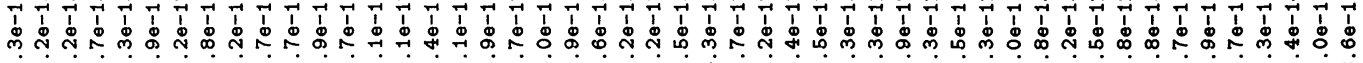

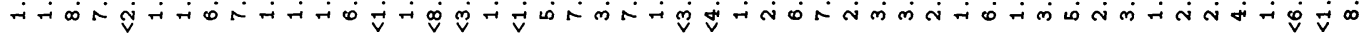

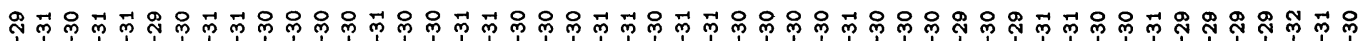

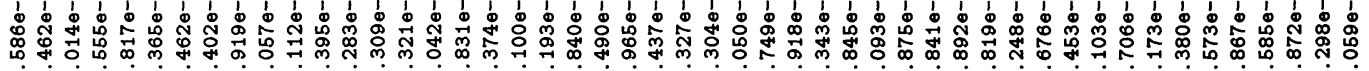

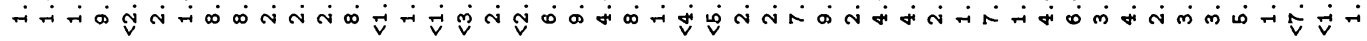

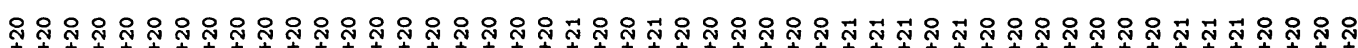

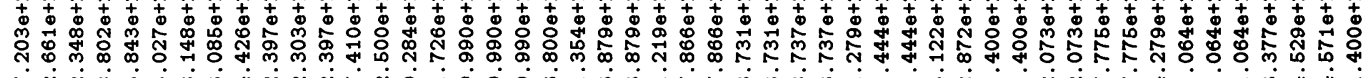

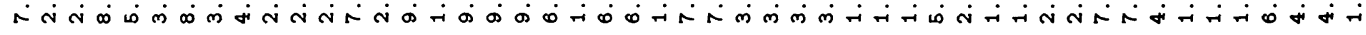

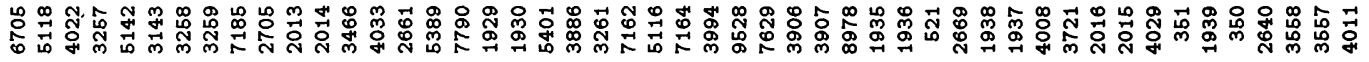

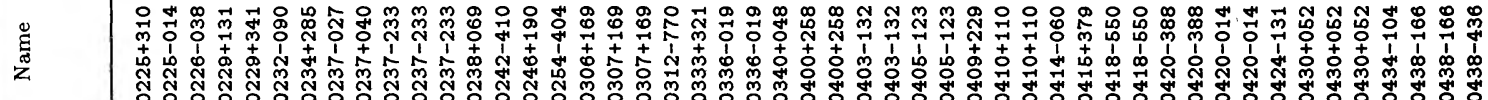


ละ

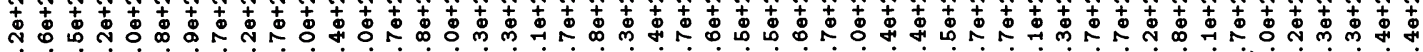
تं

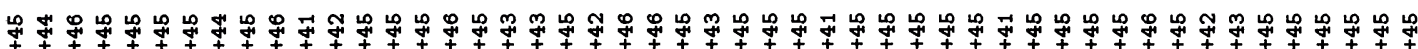

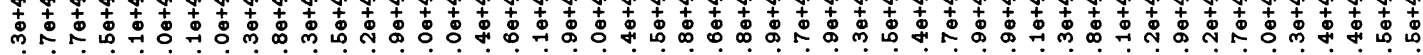

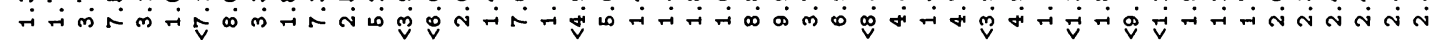

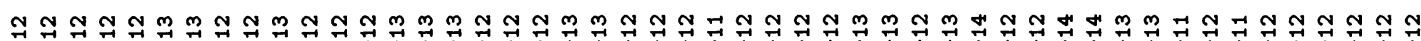

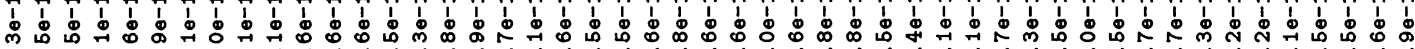
م

$\exists$ 象

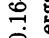
i

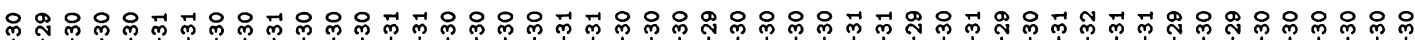

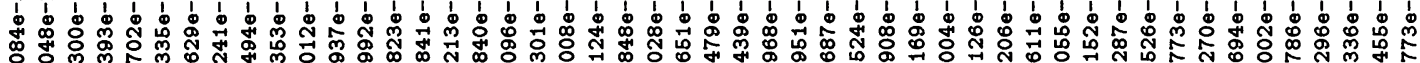

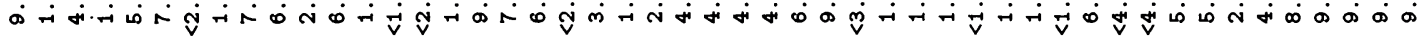

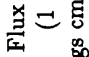

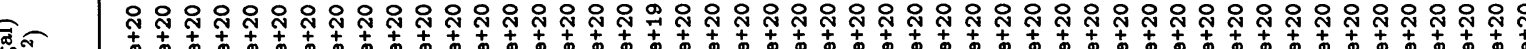

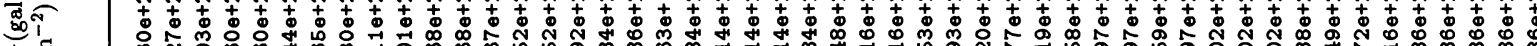

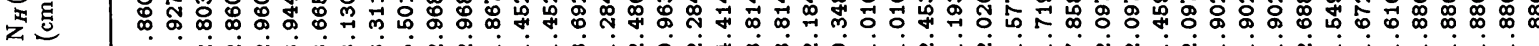

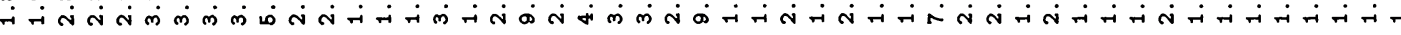

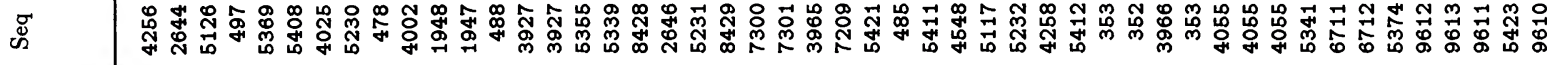

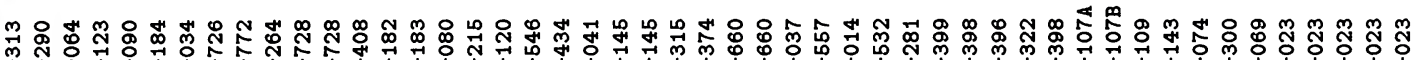

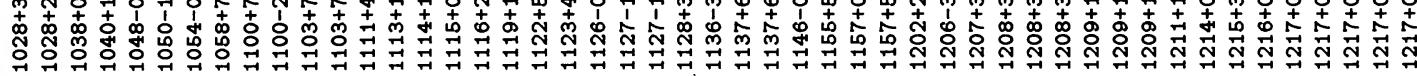

兽 คิ

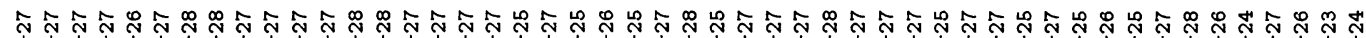

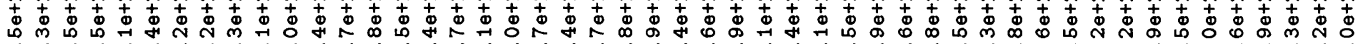
ڤ

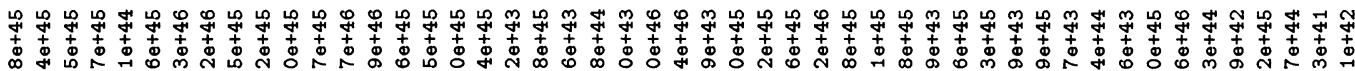

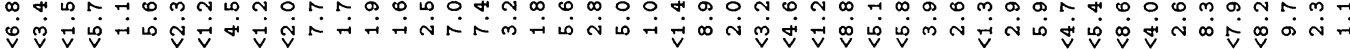

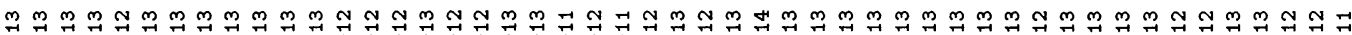

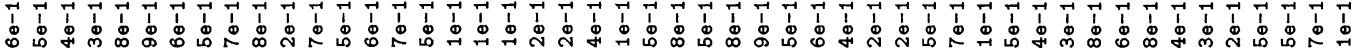

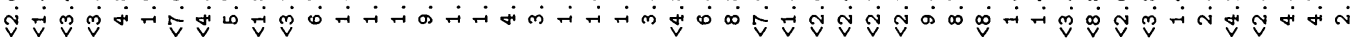

药

空

$\overbrace{\mathbb{8}}^{-1}$

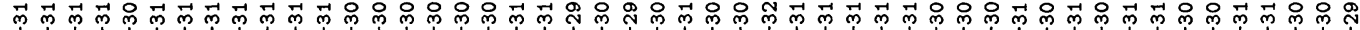

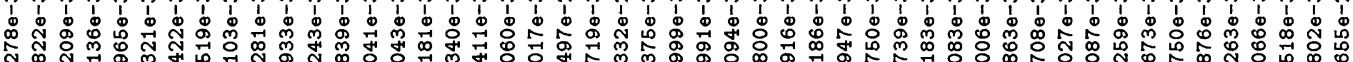

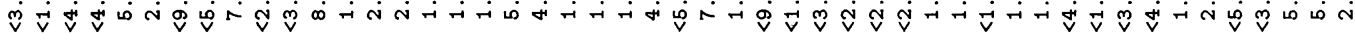

艺

m

$m m$

$m$ N

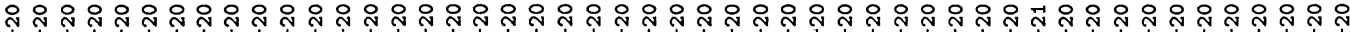

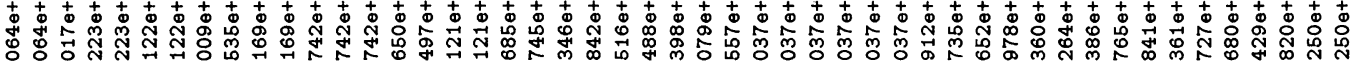

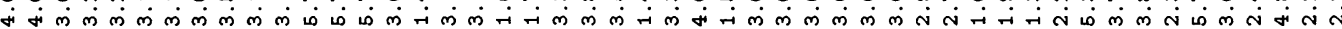

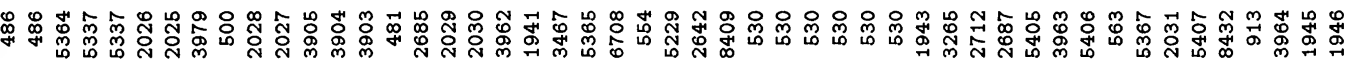

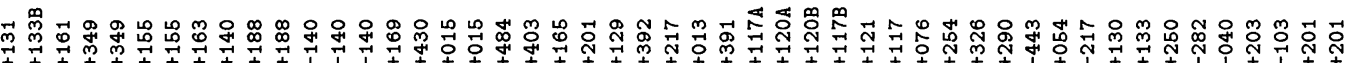

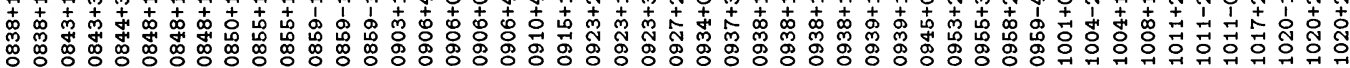




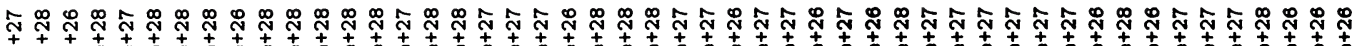

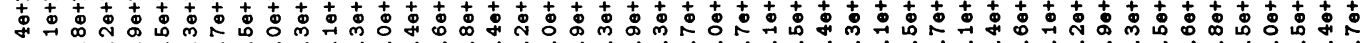

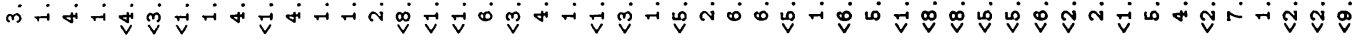

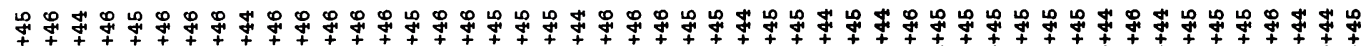

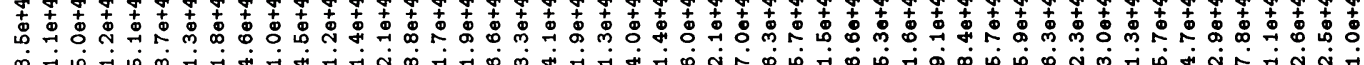
穴

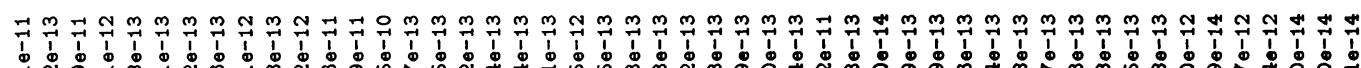
تi

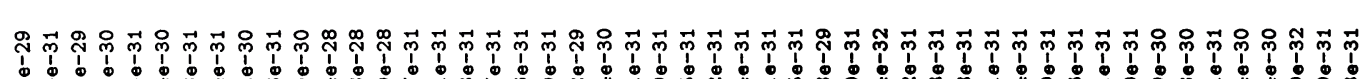

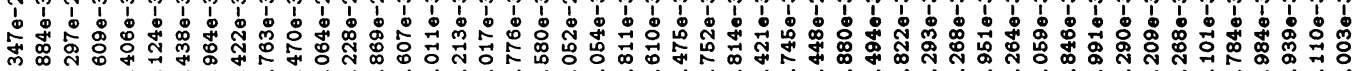
-

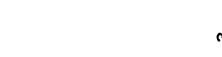

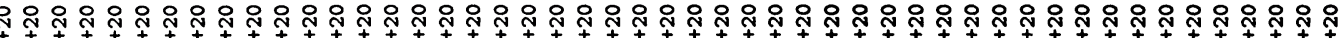

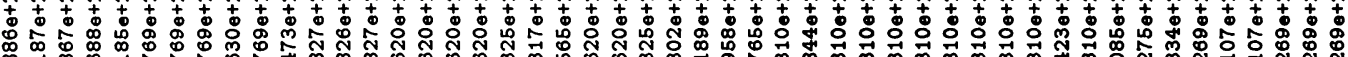

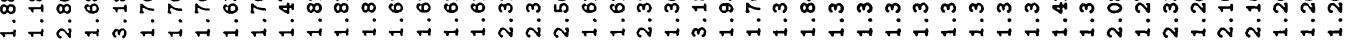

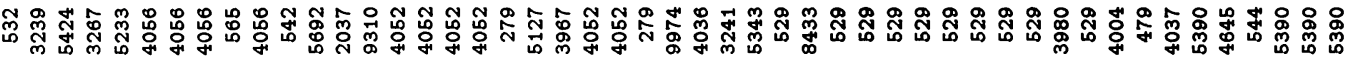

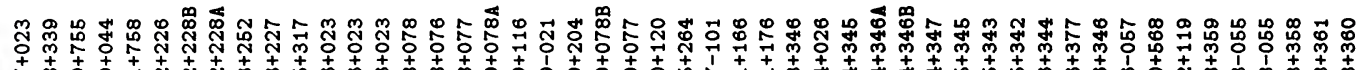

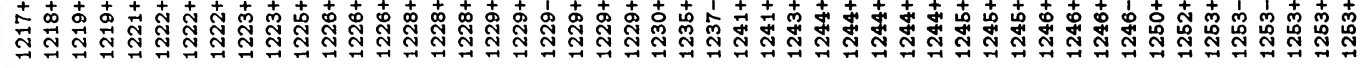

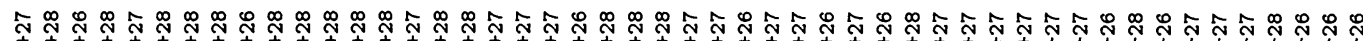

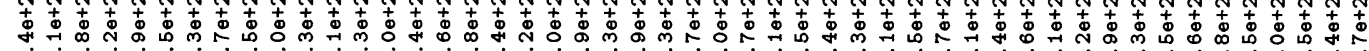

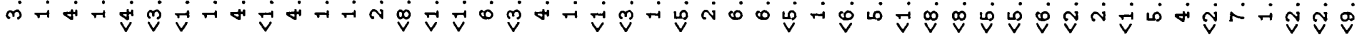

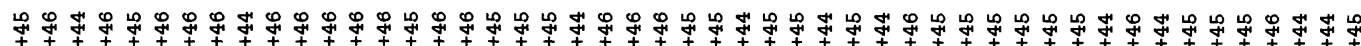

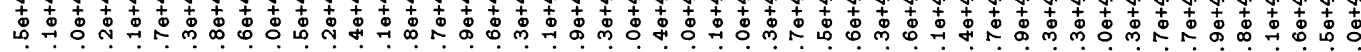
m

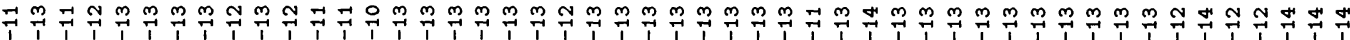

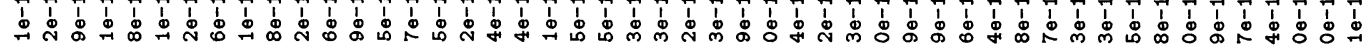
-R

$\stackrel{5}{\stackrel{1}{*}}$

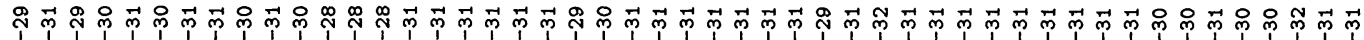

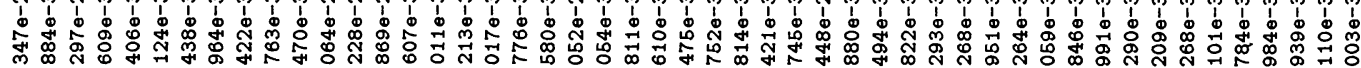
-

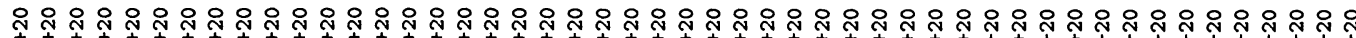

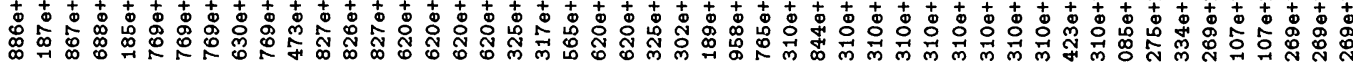

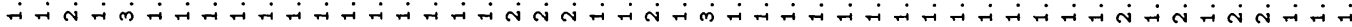

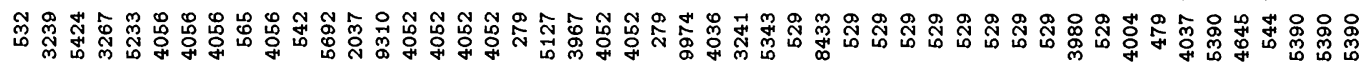

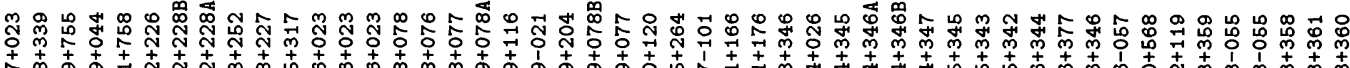

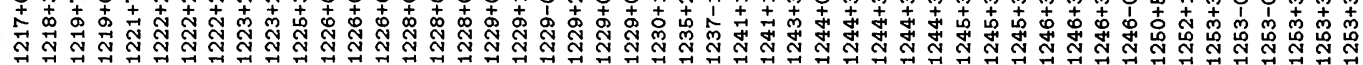




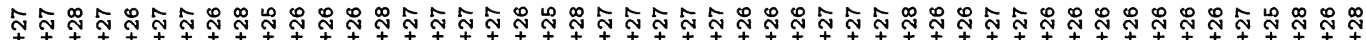

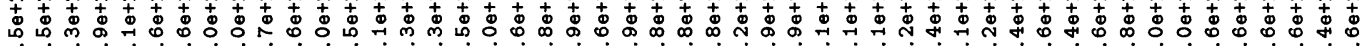

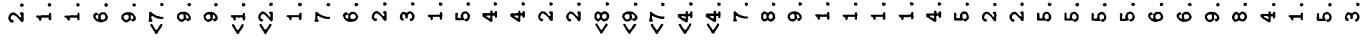

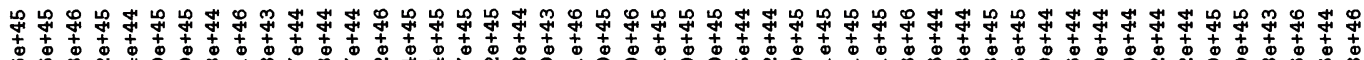
ن

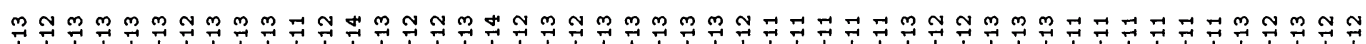

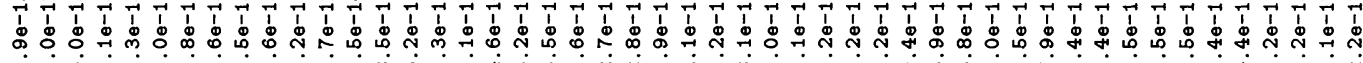
ம்

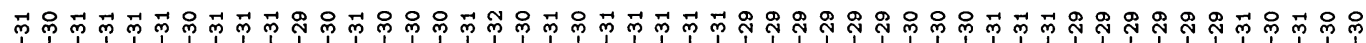

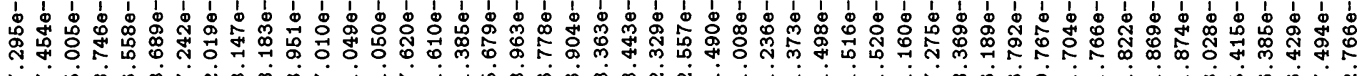

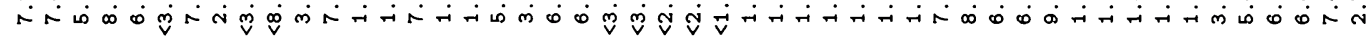

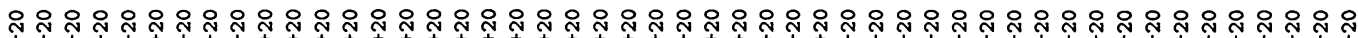

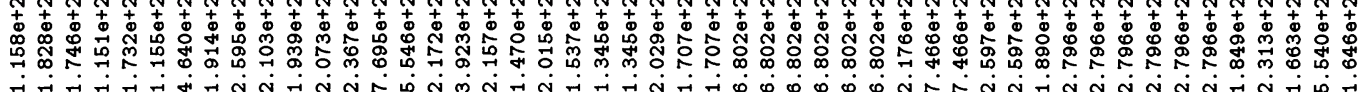

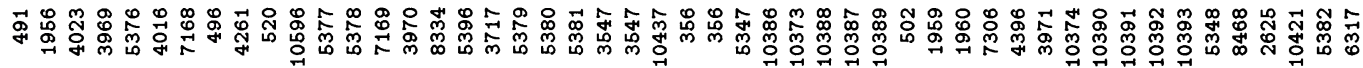

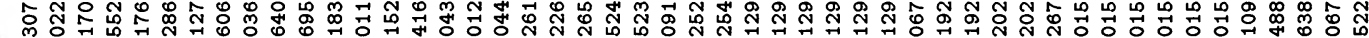

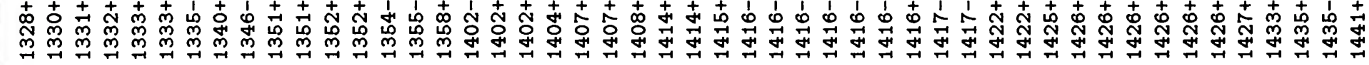

แลกิ

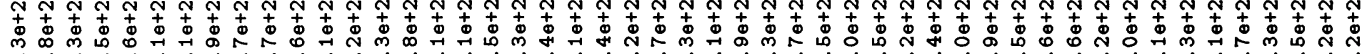
نु

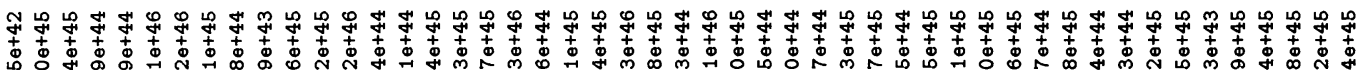
पु

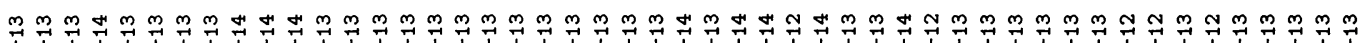

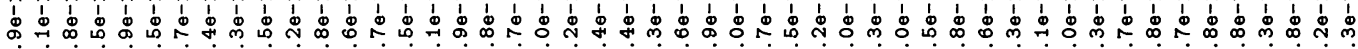

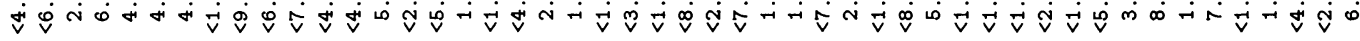

$\vec{p} \vec{p} \vec{p} \tilde{p} \vec{p} \vec{p} \vec{p} \vec{p} \vec{p} \tilde{p} \tilde{p} \vec{p} \vec{p} \vec{p} \vec{p} \vec{p} \vec{p} \vec{p} \vec{p} \vec{p} \vec{p} \vec{p} \vec{p} \vec{p} \vec{p} \vec{p} \tilde{p} \tilde{p}$

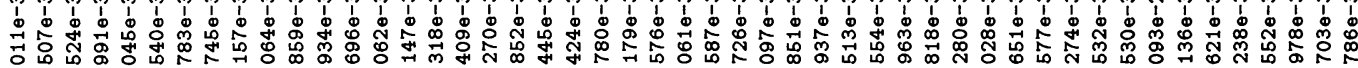

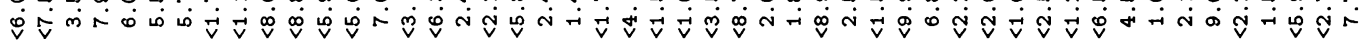

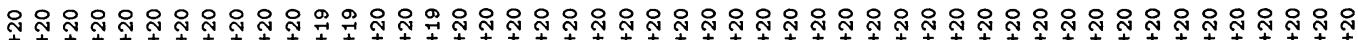

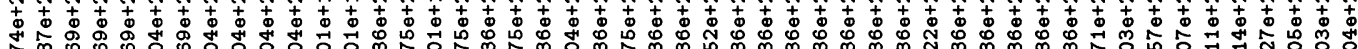

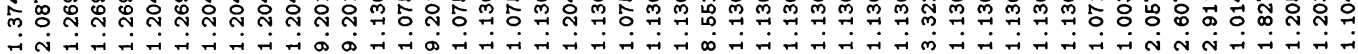

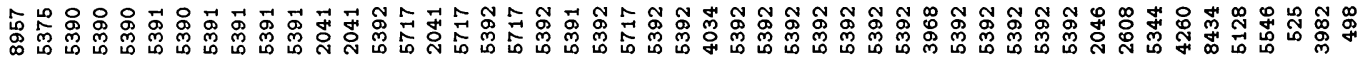

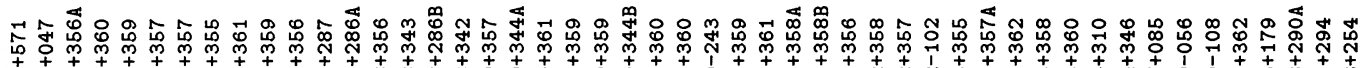

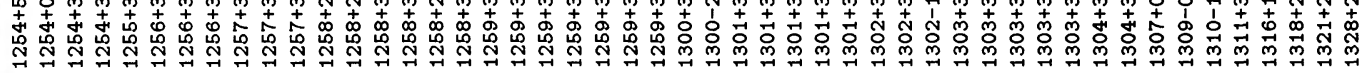




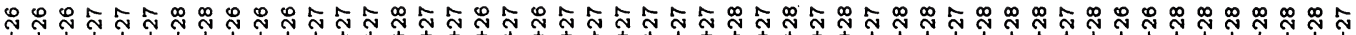

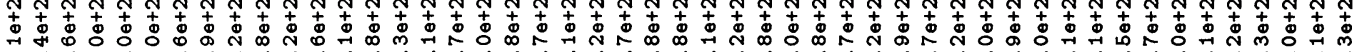

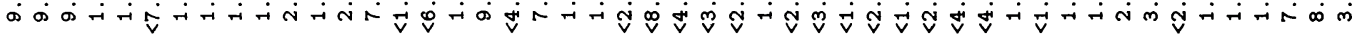

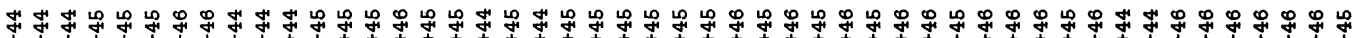

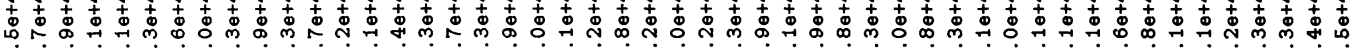

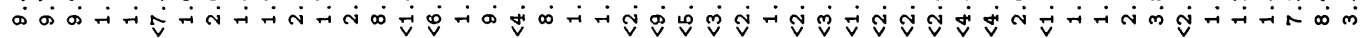

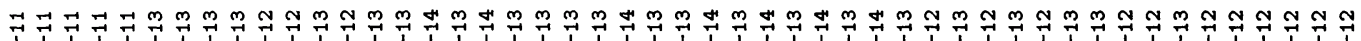

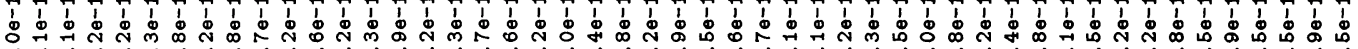

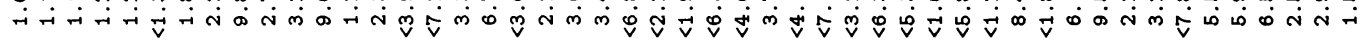

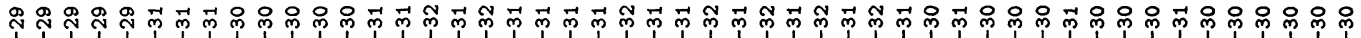

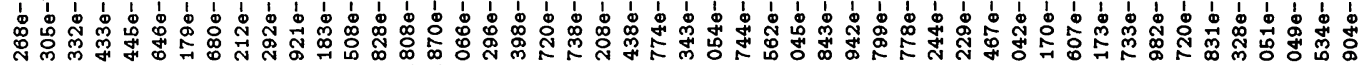

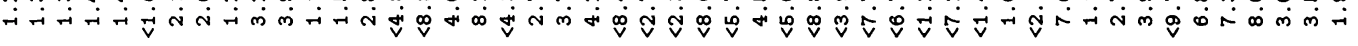

$m$

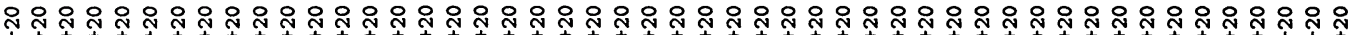

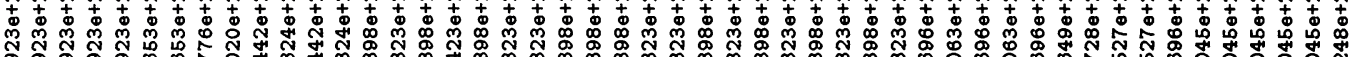
i $\dot{\sim}$ i

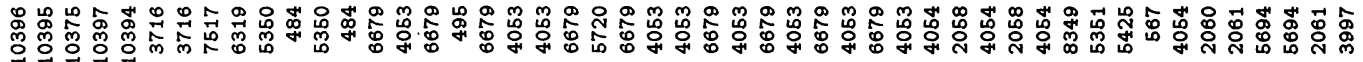

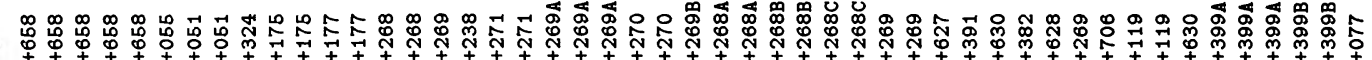

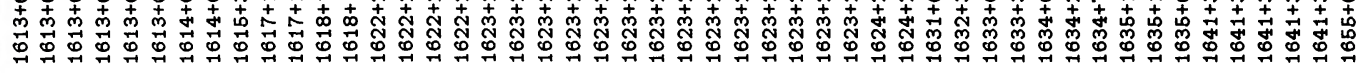

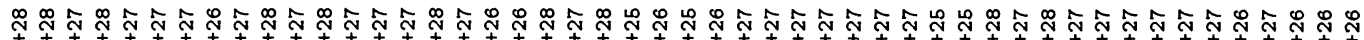

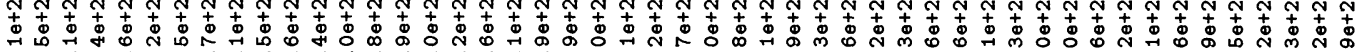

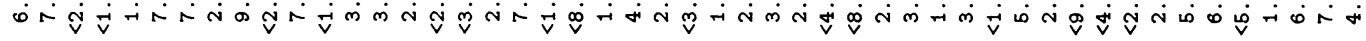

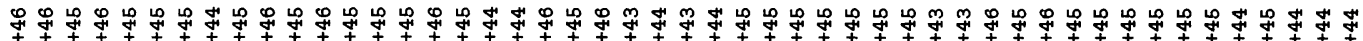

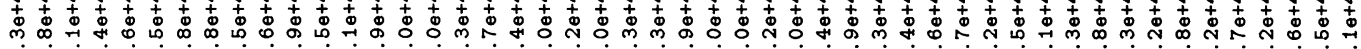

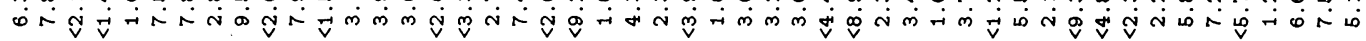

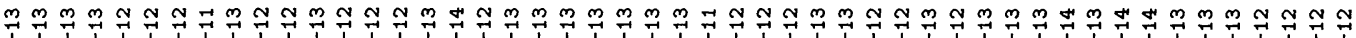

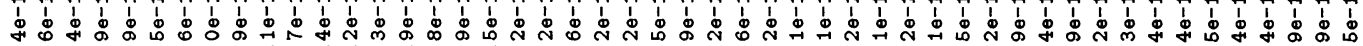
ம

$\vec{p} \vec{p} \vec{p} \vec{p}$ 웅유

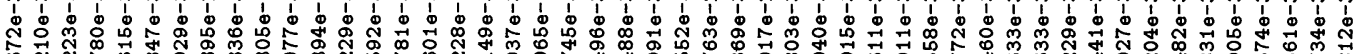
i

$$
\text { m }
$$

$m m$

m

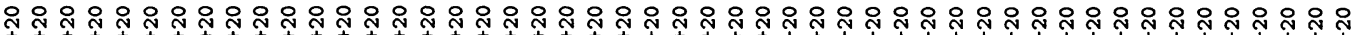

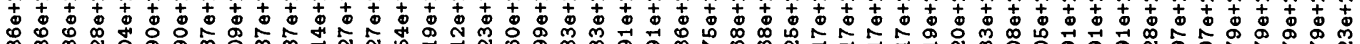
N न न

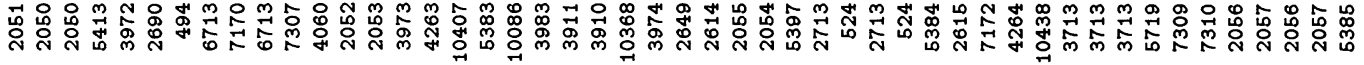

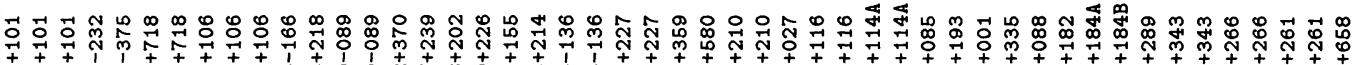

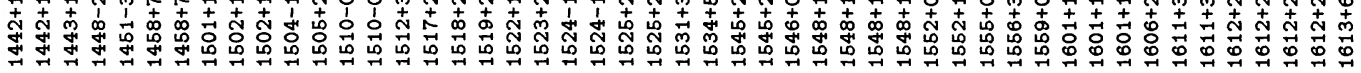




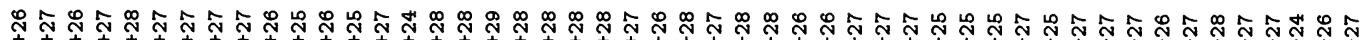

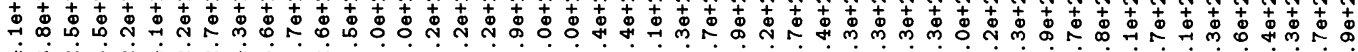

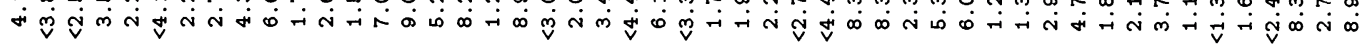

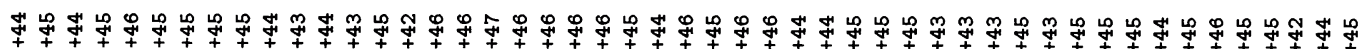

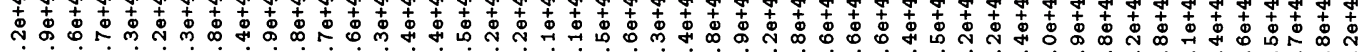
ن

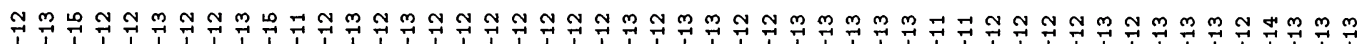

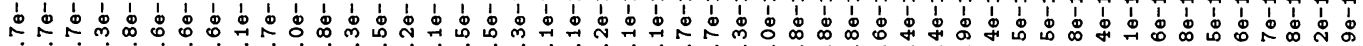

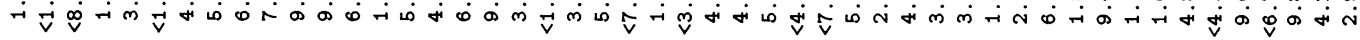

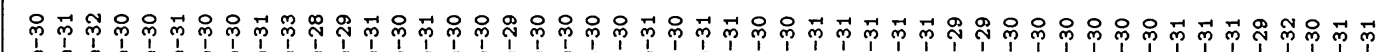

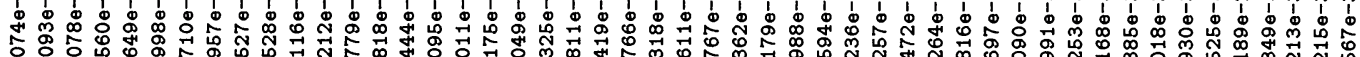

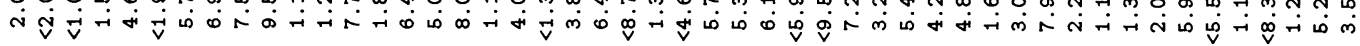

$m \rightarrow \quad m$

$m$

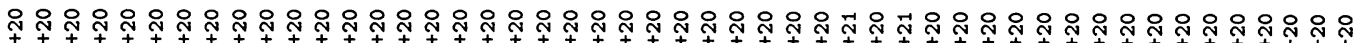

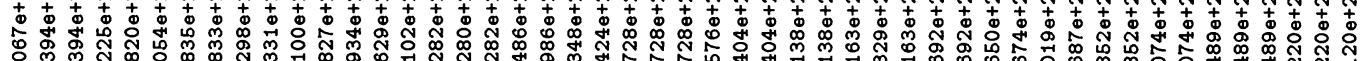

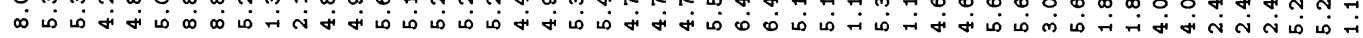

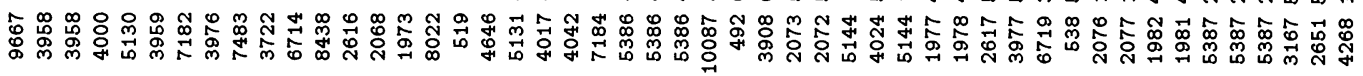

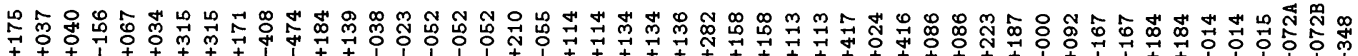

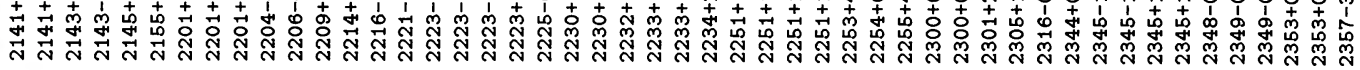

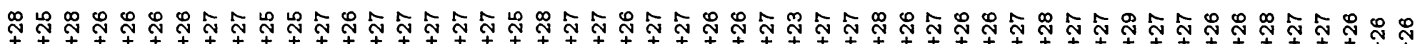

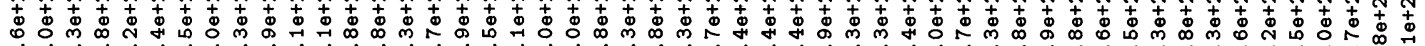

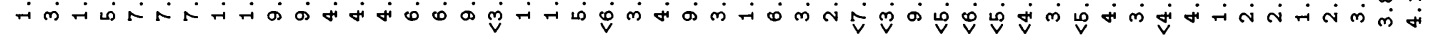

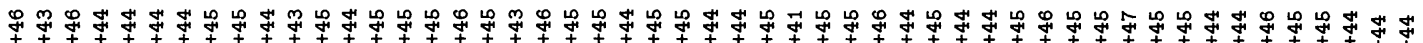

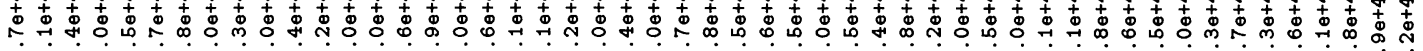

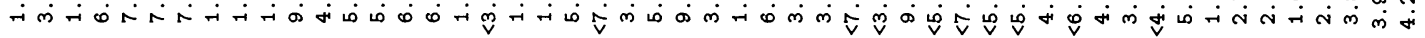

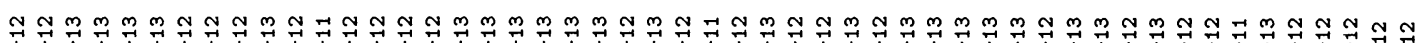

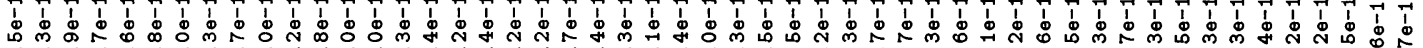

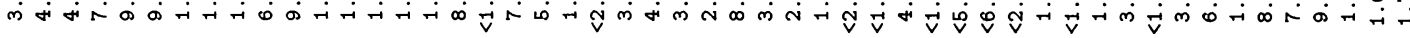

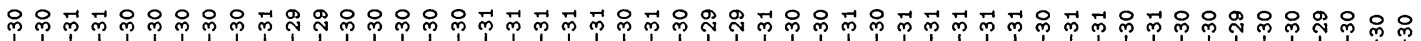

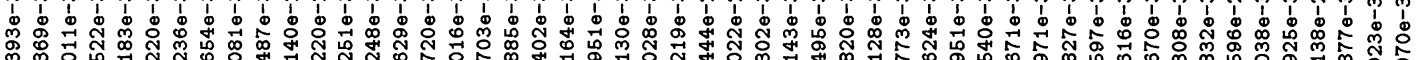
अ ம்

m $m$

$m$

$m$

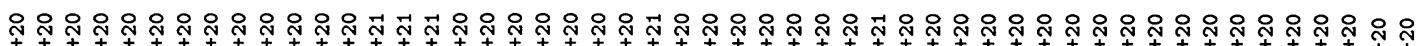

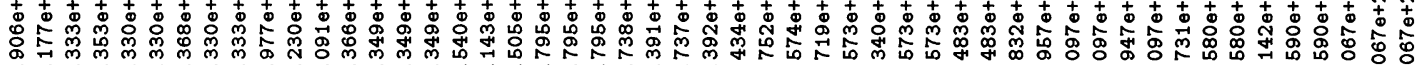

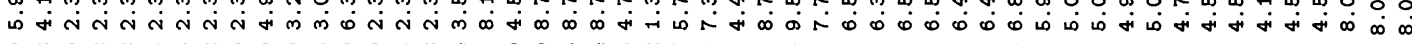

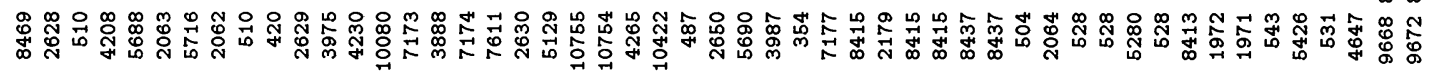

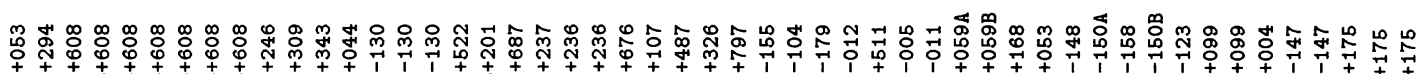

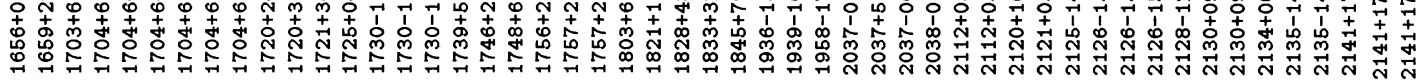




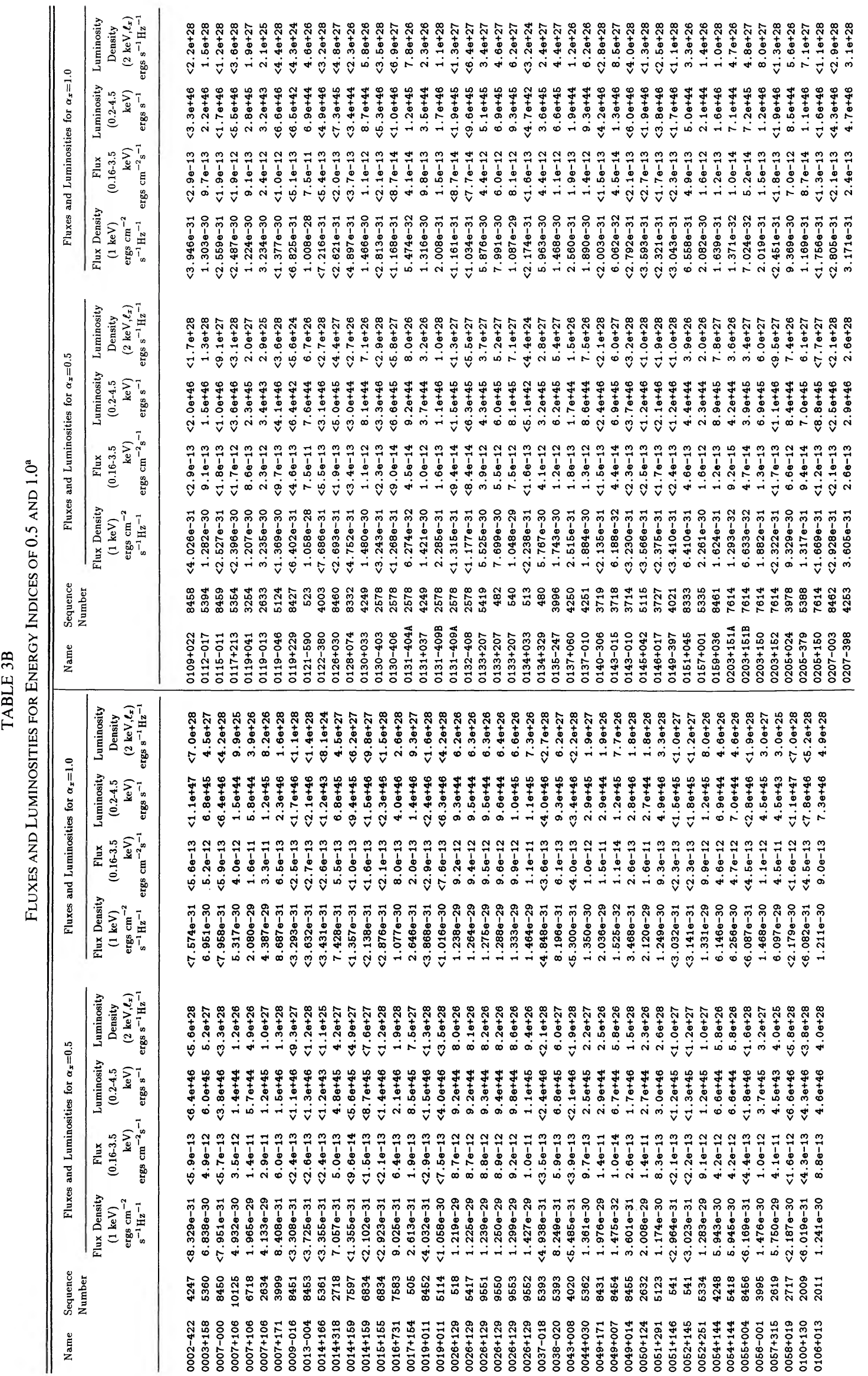




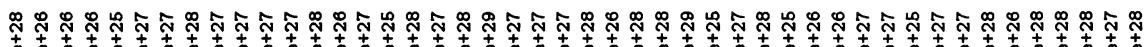

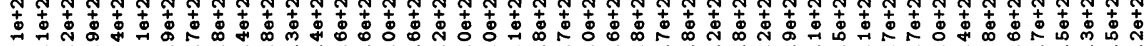

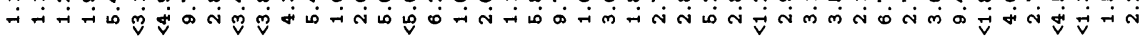

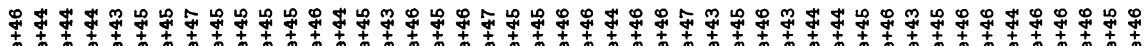

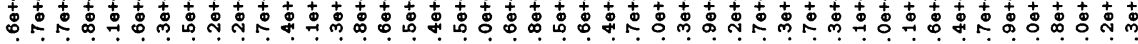
नित

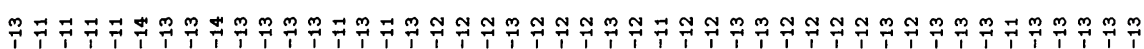

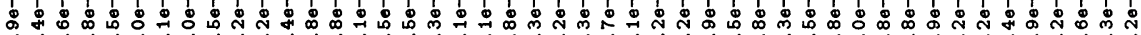

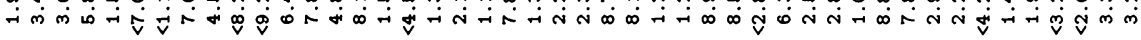

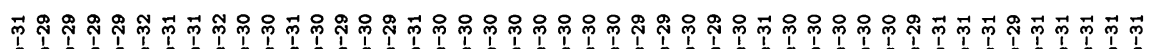

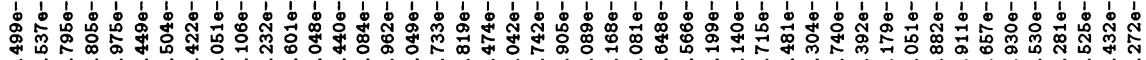
ن में

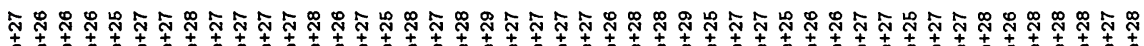

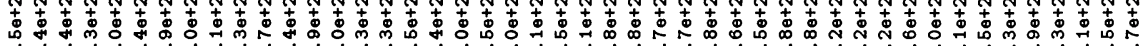

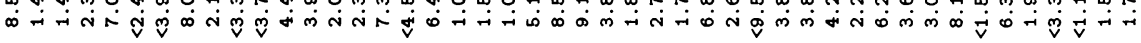

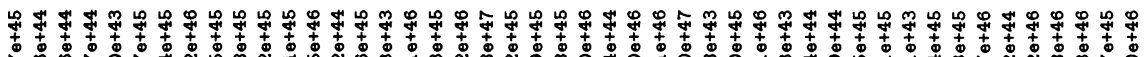
ه D

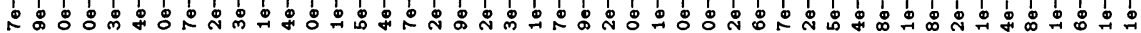

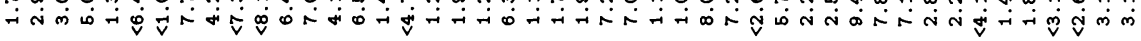

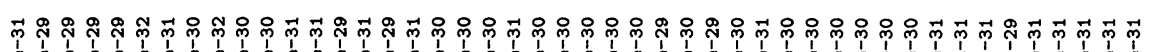

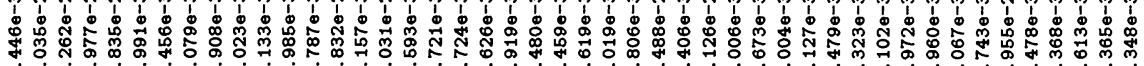

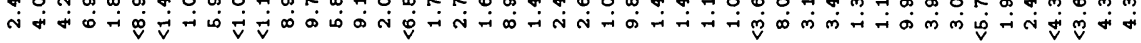

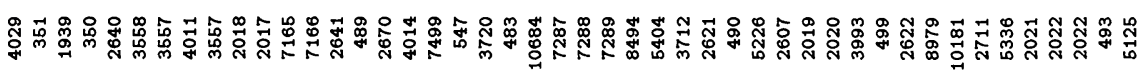

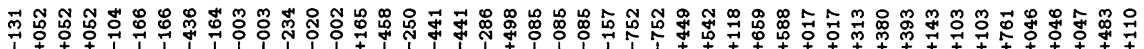

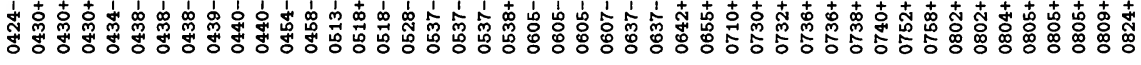

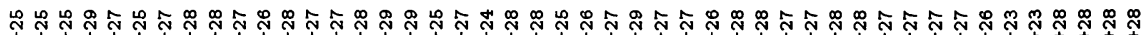

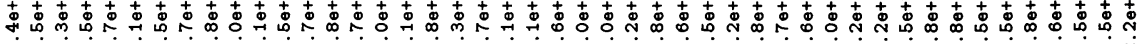

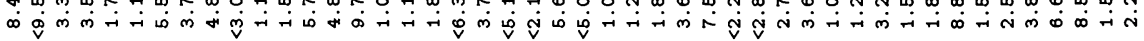

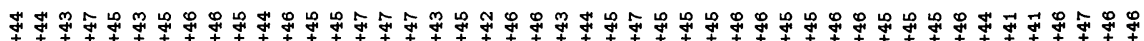

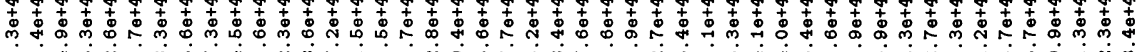
-

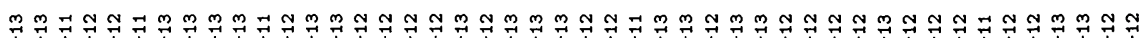

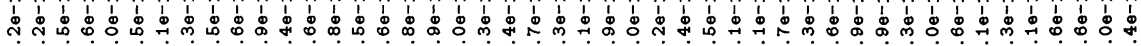
人娄

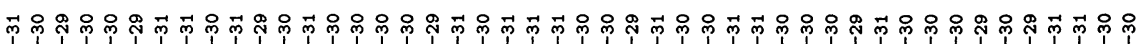

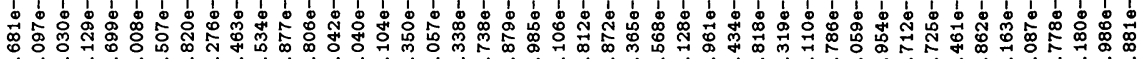

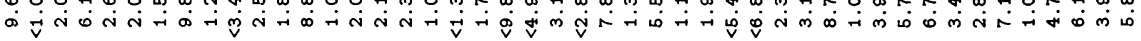

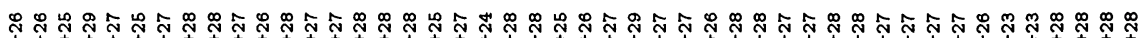

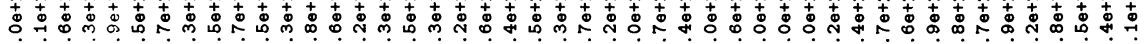
†ि

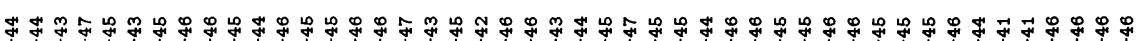

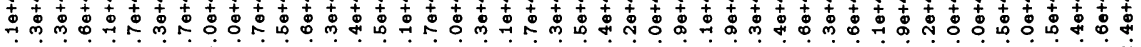

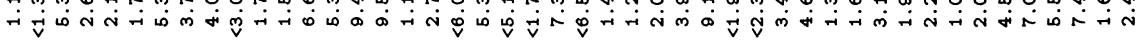

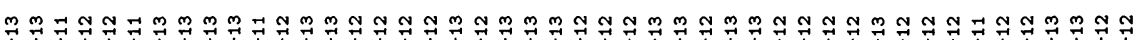

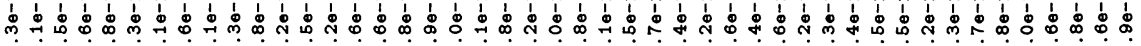
过仙

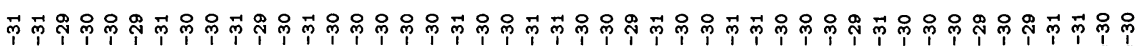

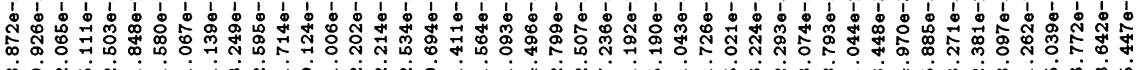

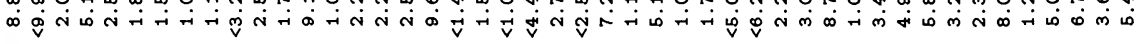

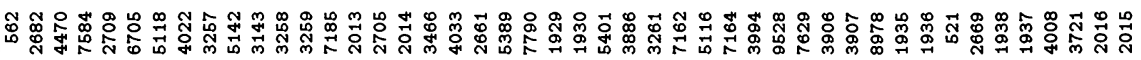

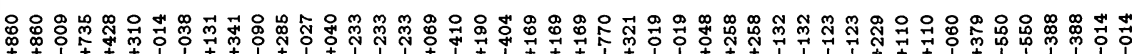

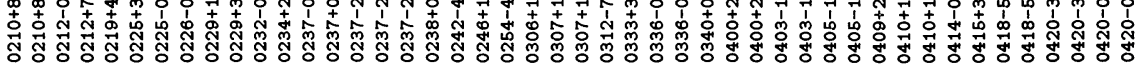




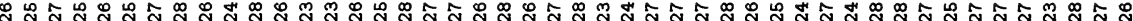

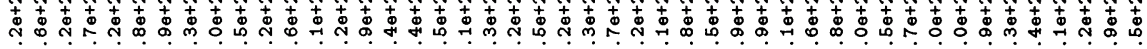

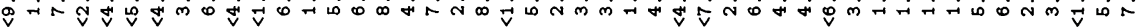

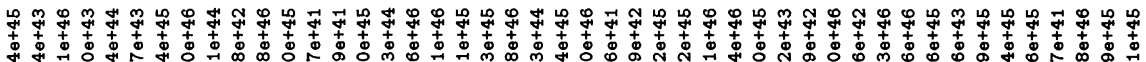
पित में

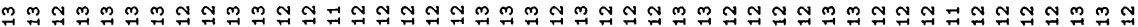
等

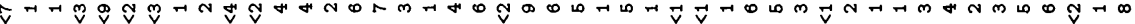

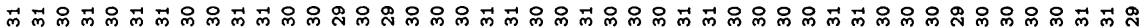

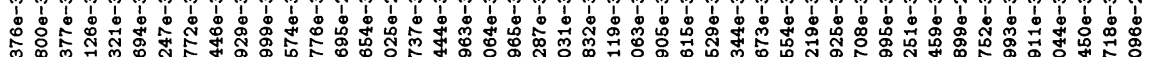

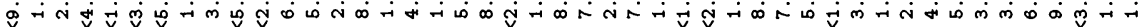

ลับิง

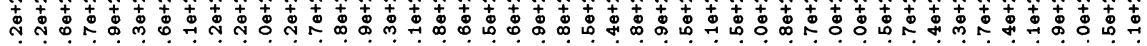

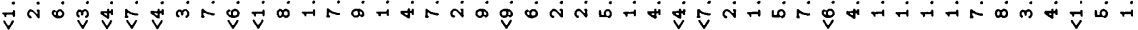

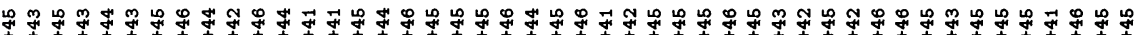

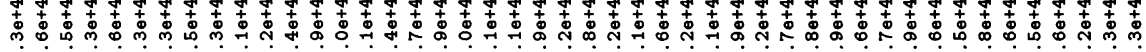

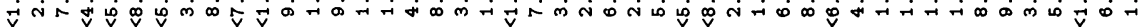
m

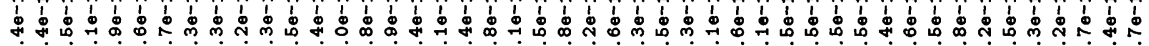

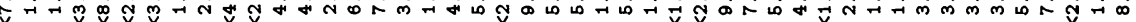

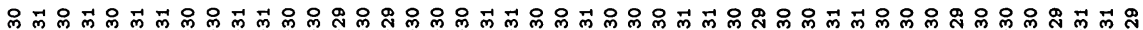

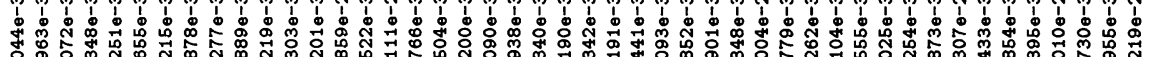

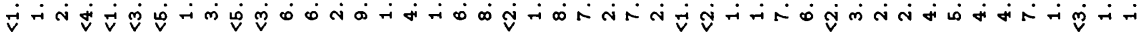

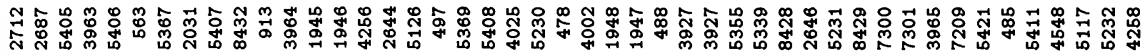

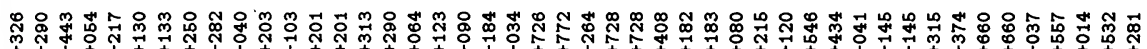

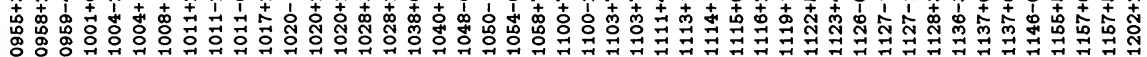

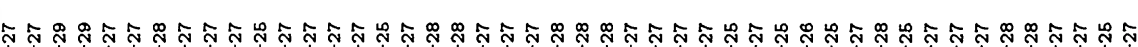

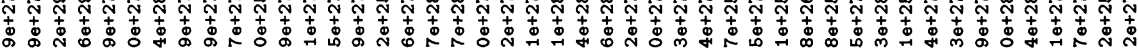

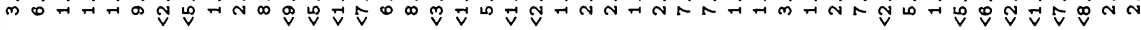

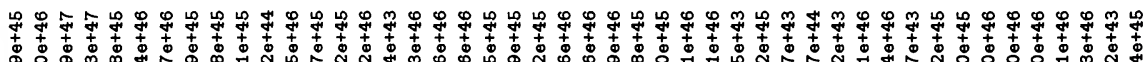

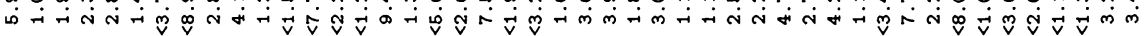

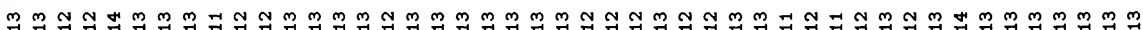

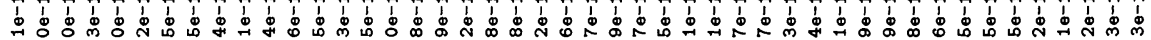

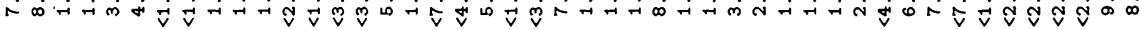

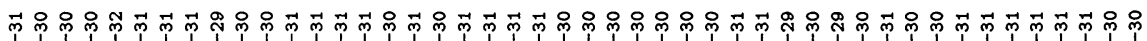

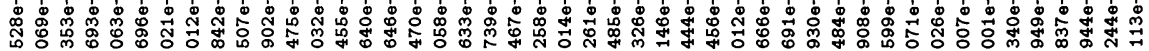

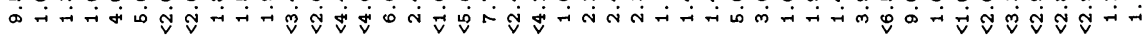

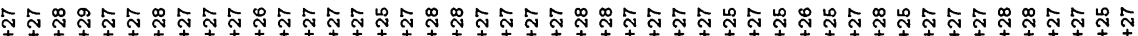

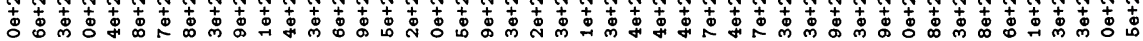

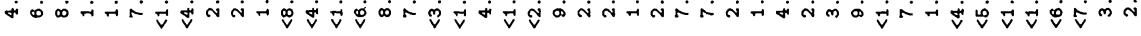

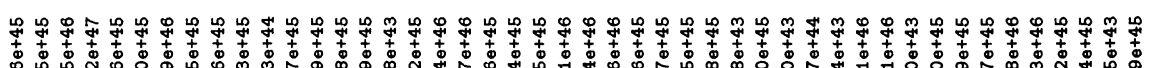

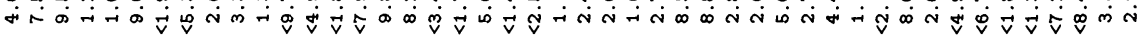

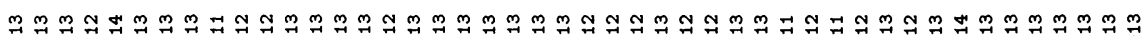

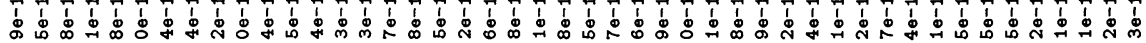

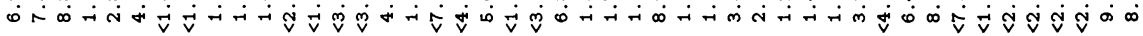

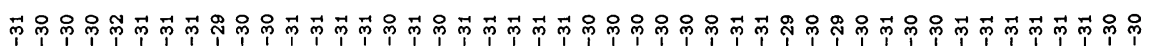

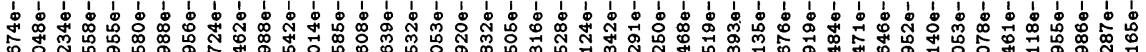

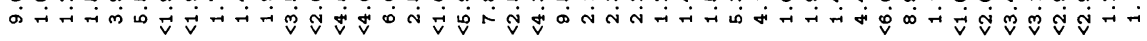

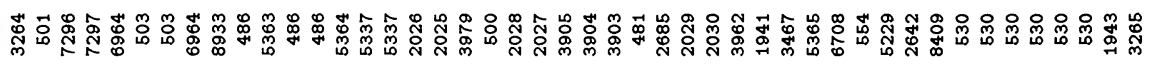

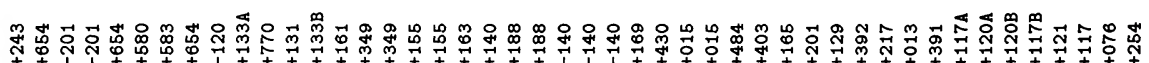

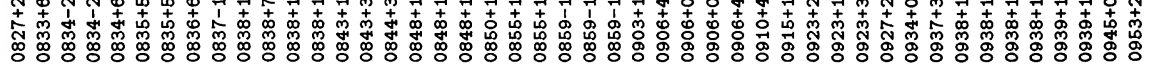




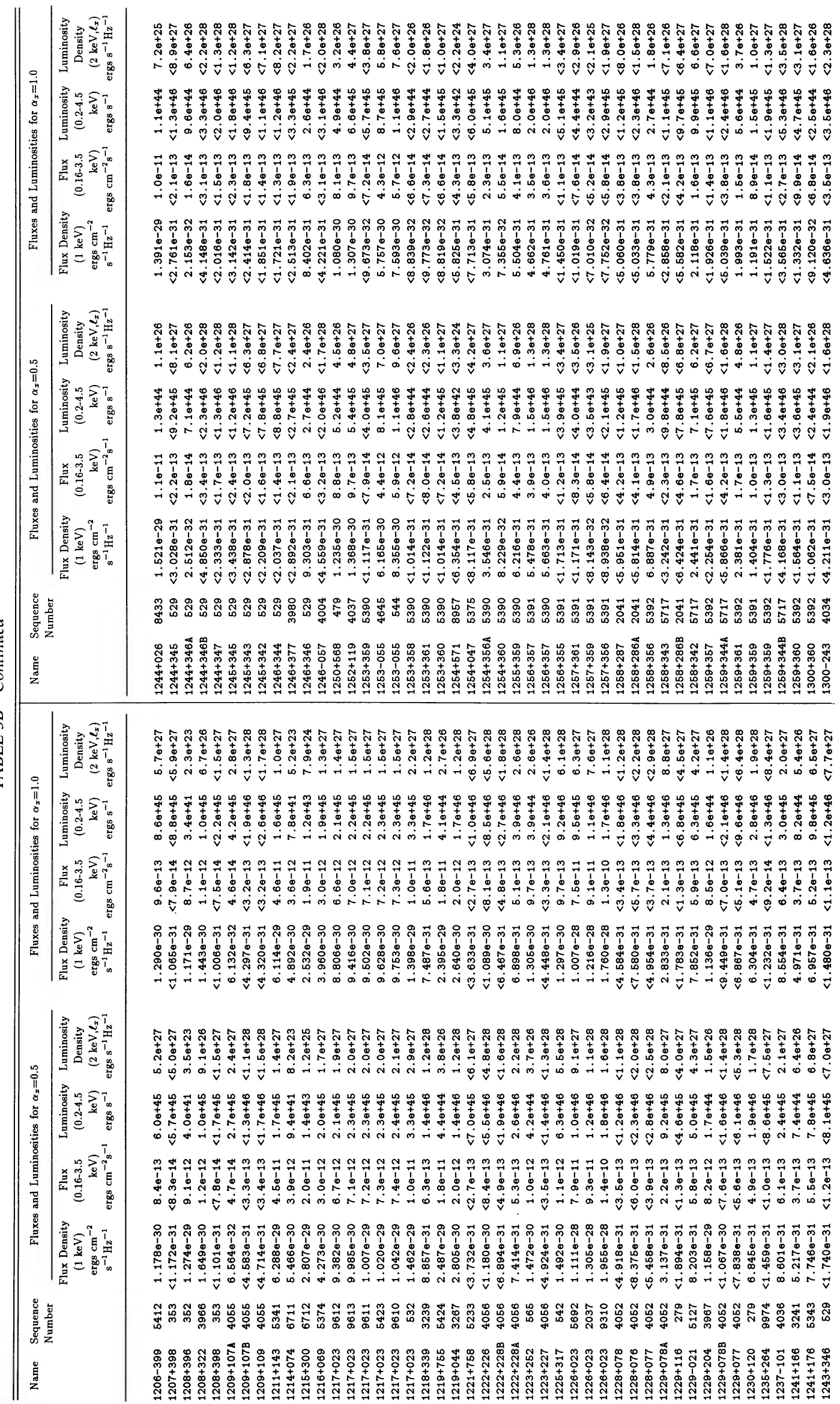




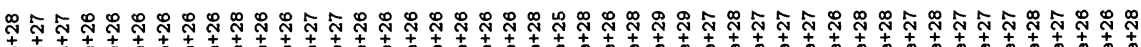

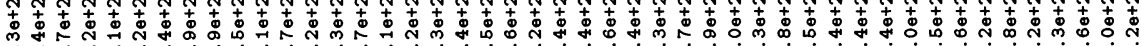

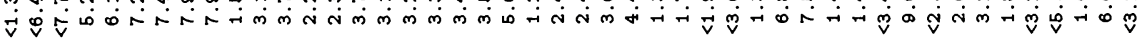

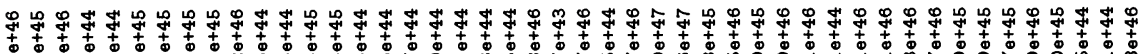
نे

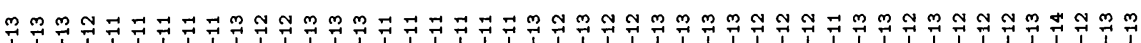

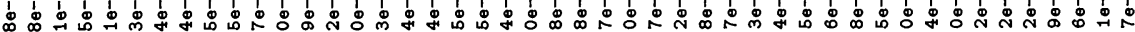

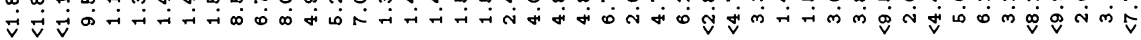

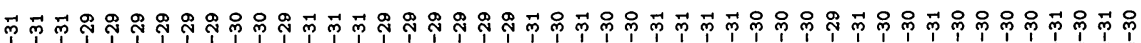

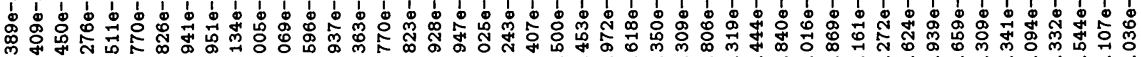

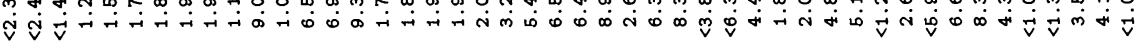

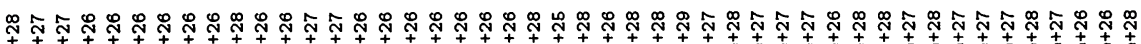

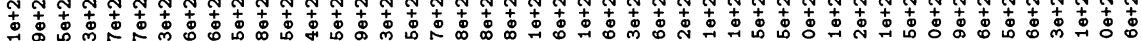

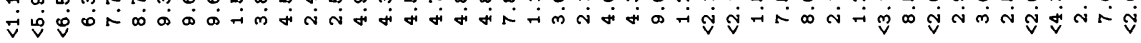

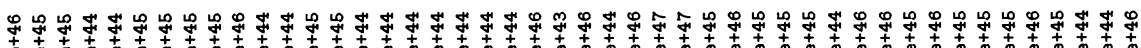

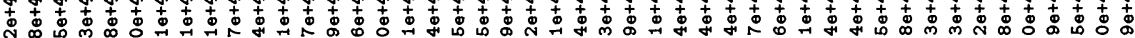

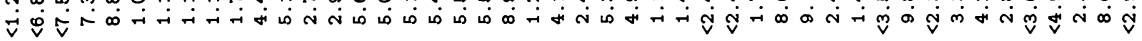

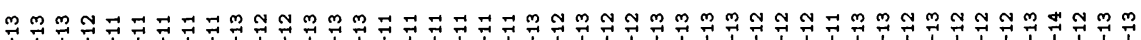

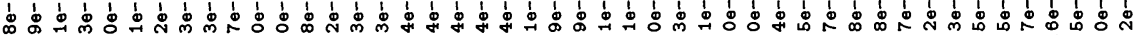

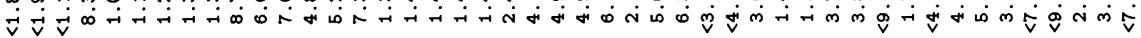

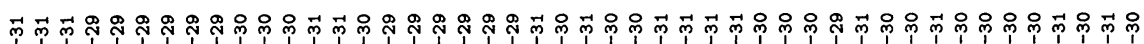

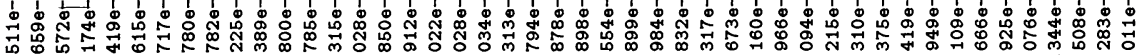

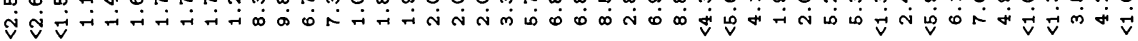

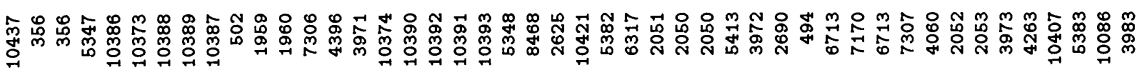

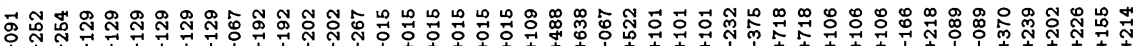

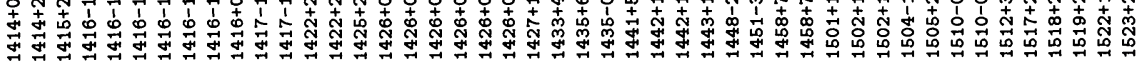

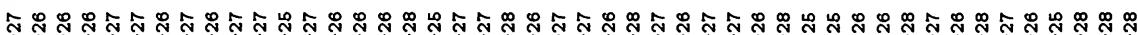

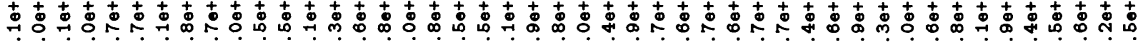

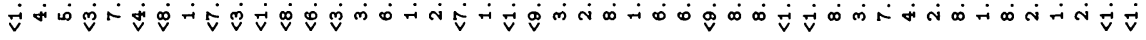

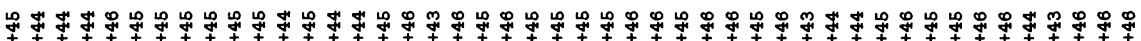

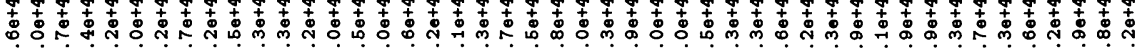

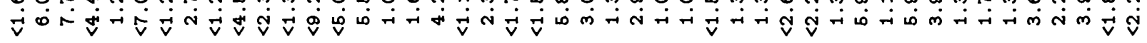

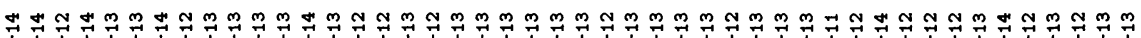

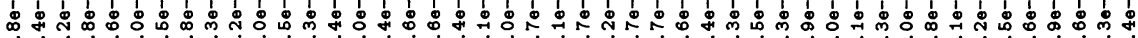

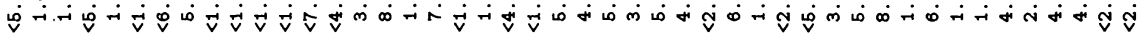

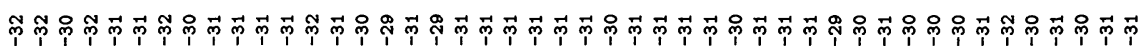

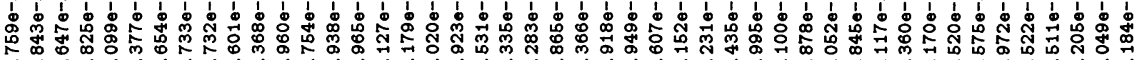

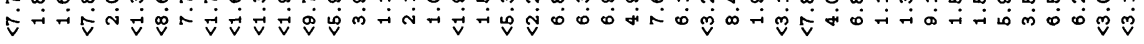

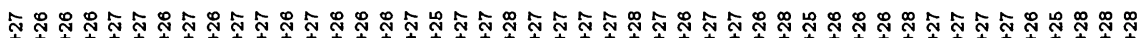

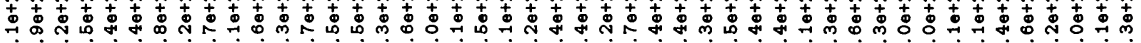

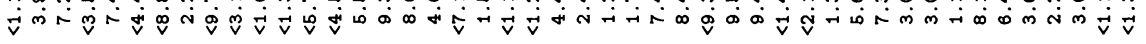

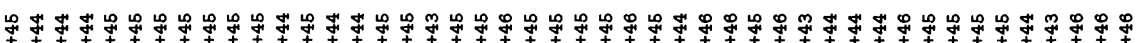

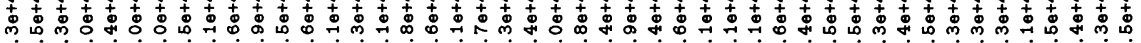

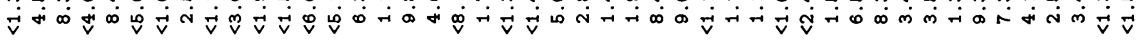

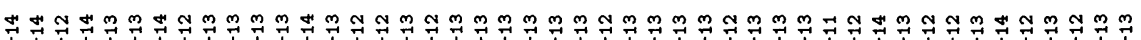

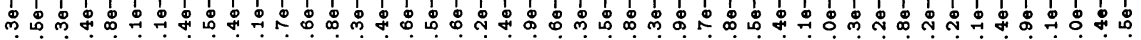

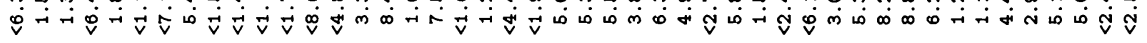

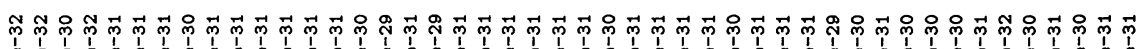

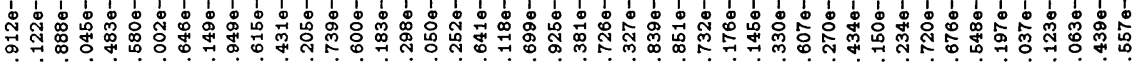

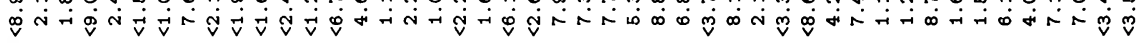

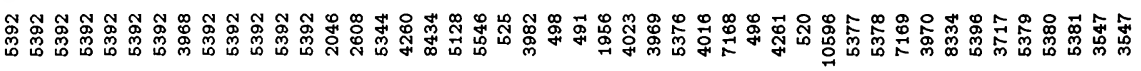

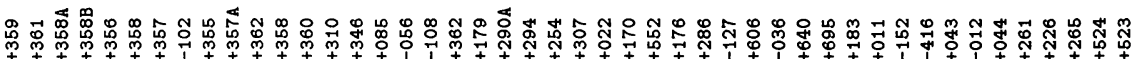

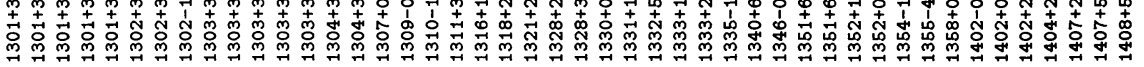

82 


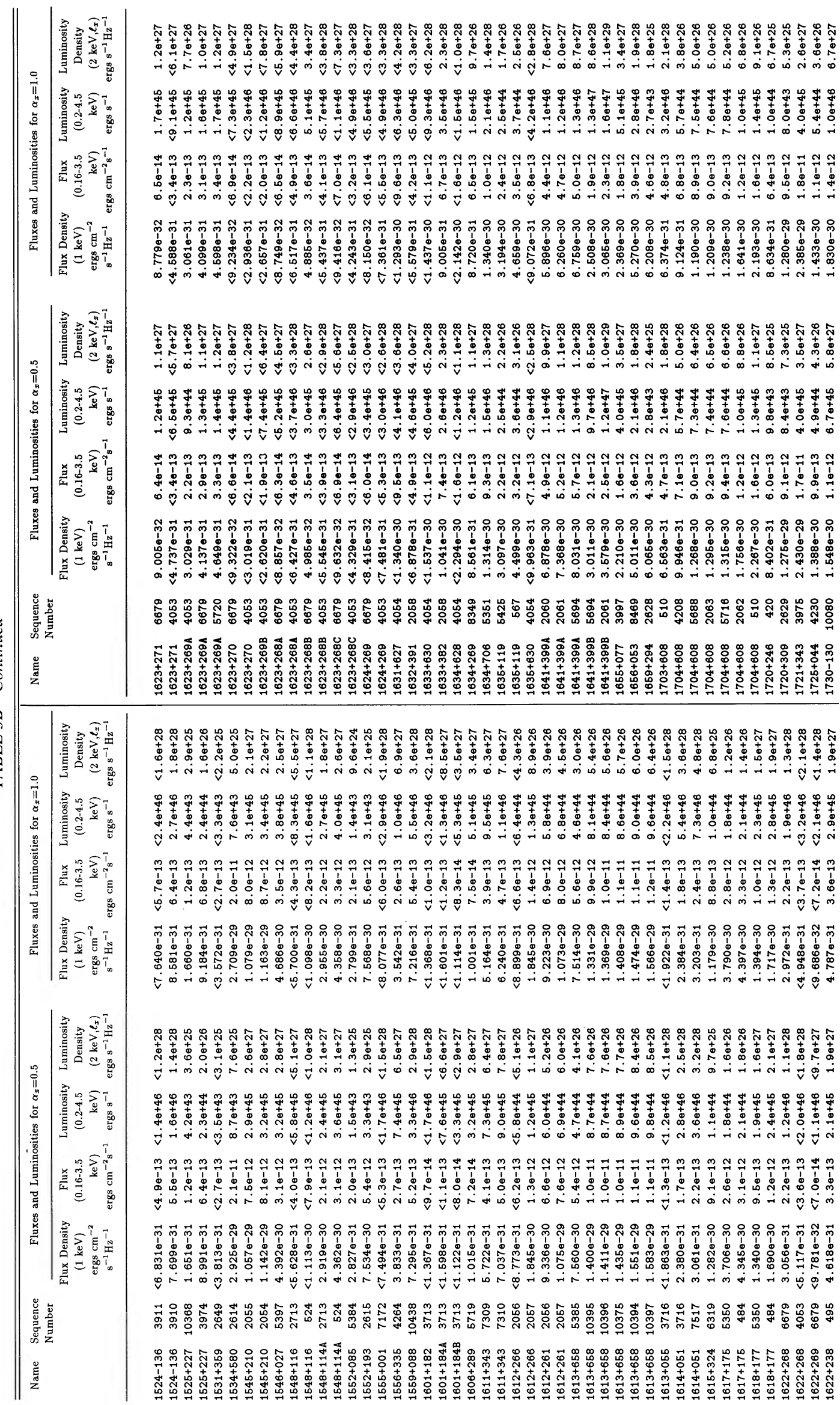

83 


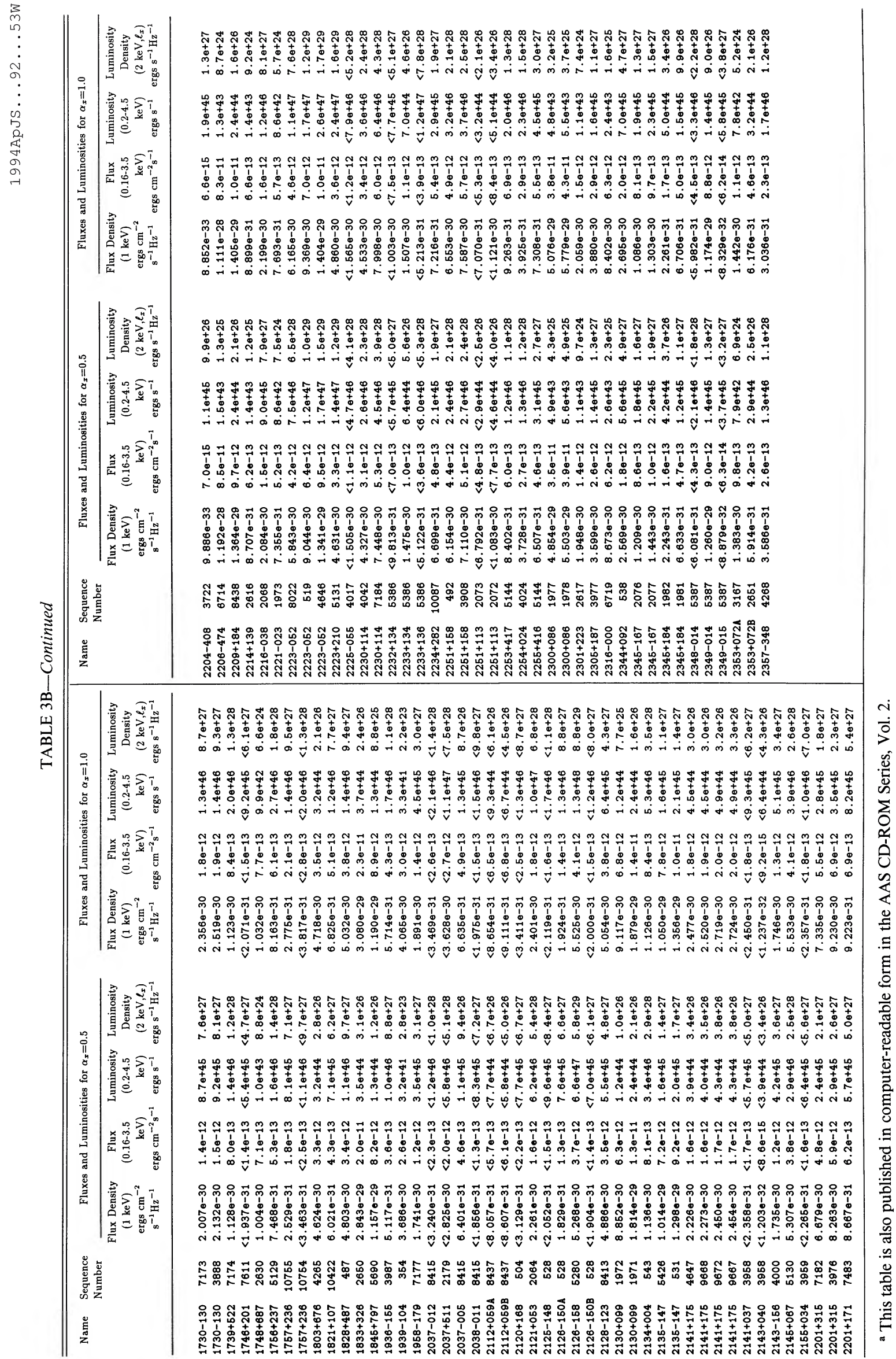

84 


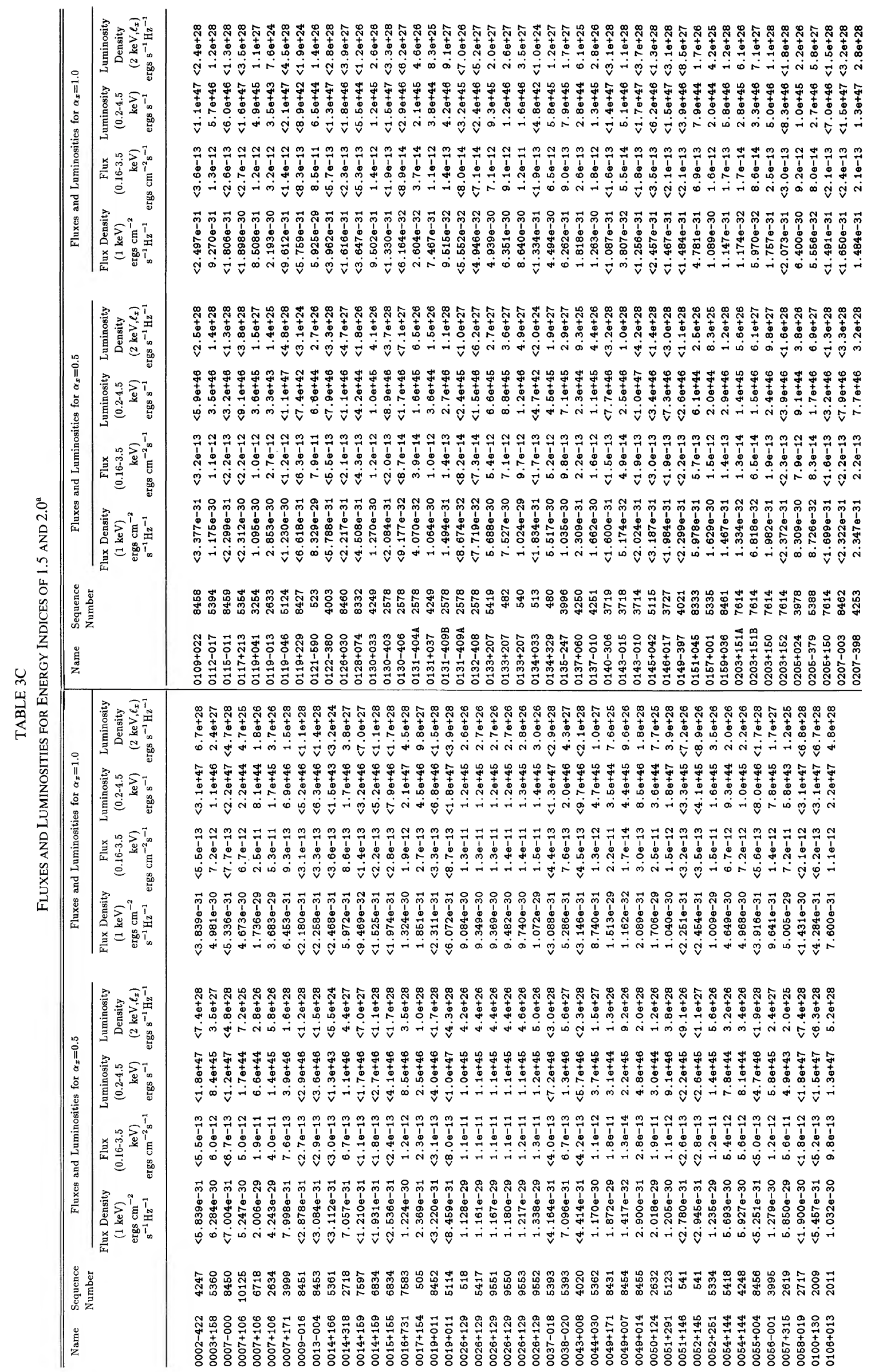




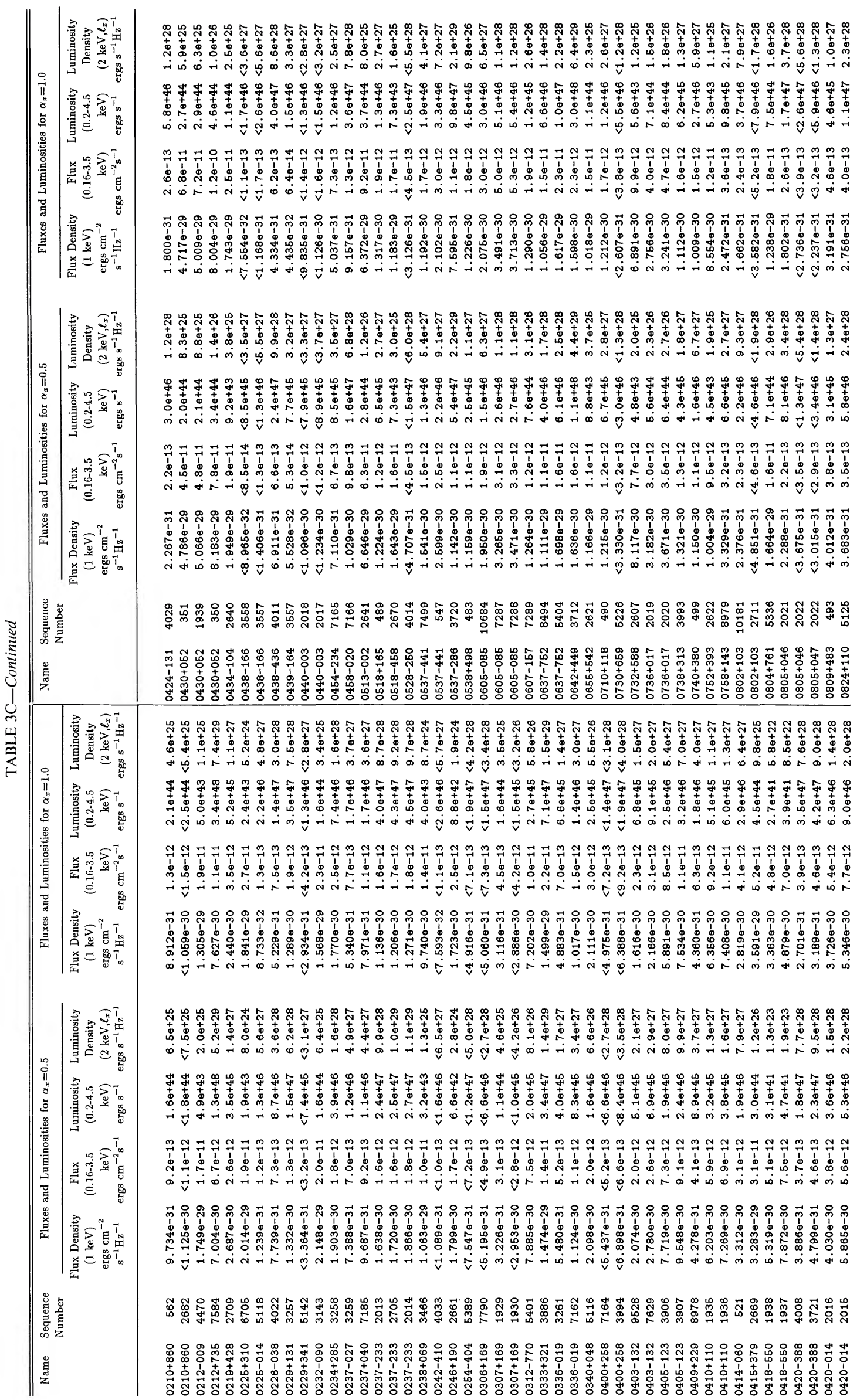




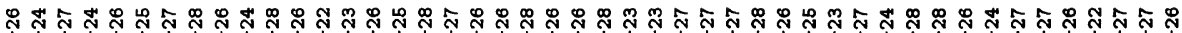

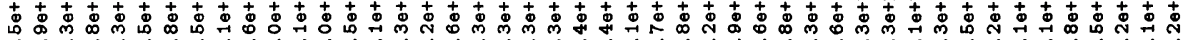

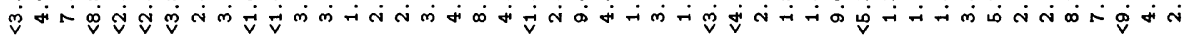

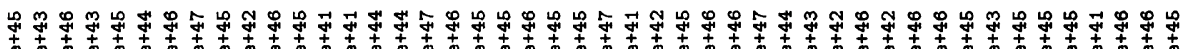

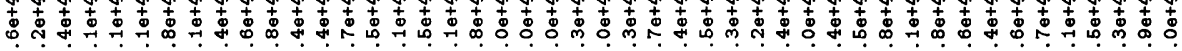

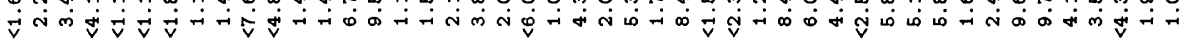
舟

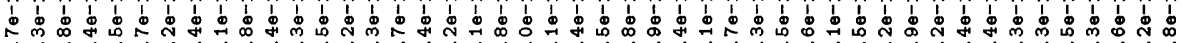

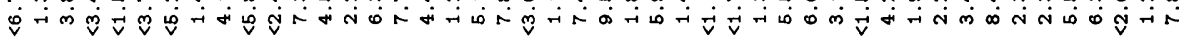

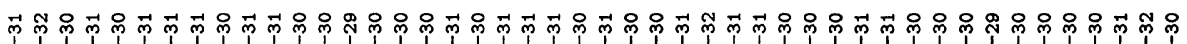

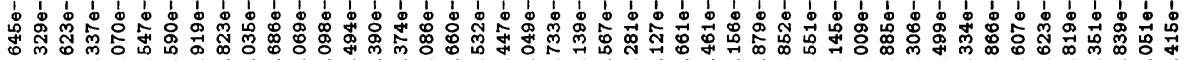
पुंक त

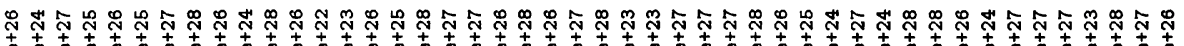

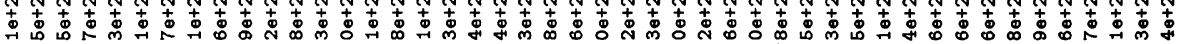

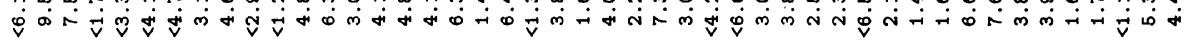

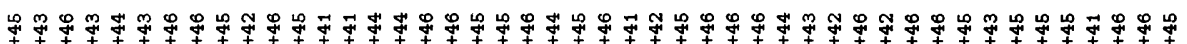

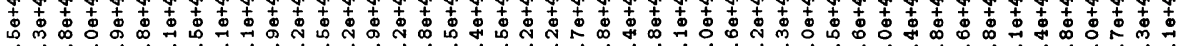

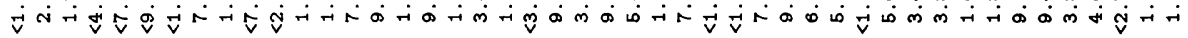

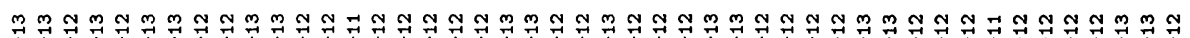

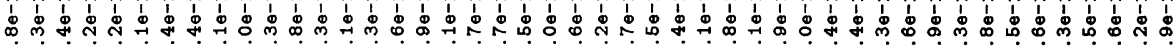

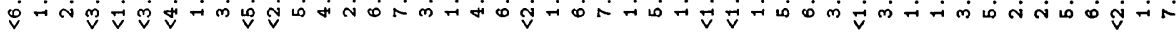

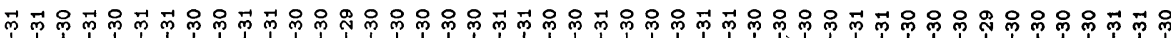

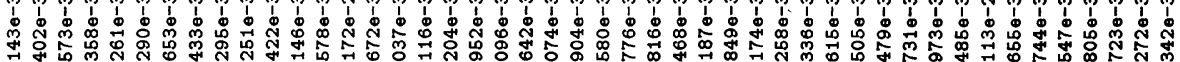

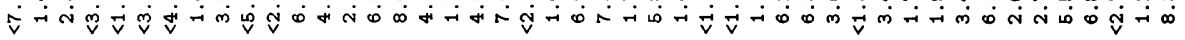

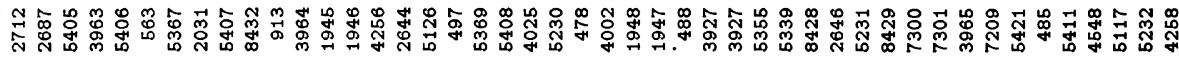

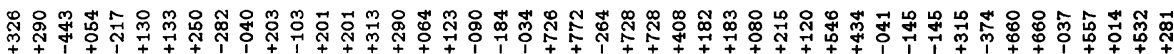

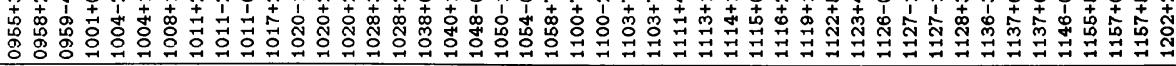

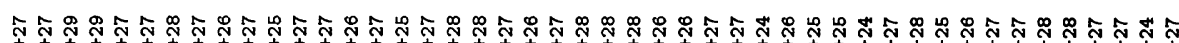

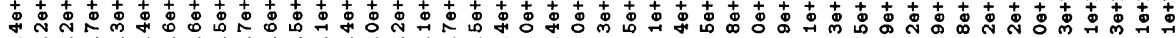
نं

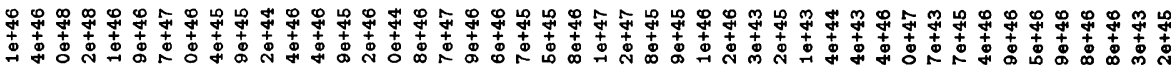

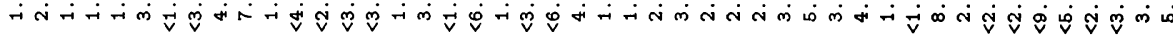

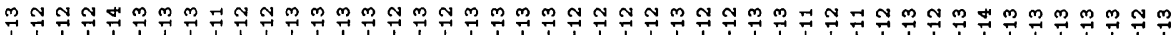

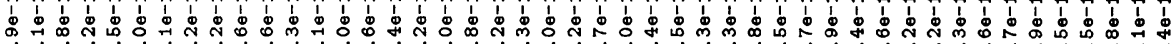

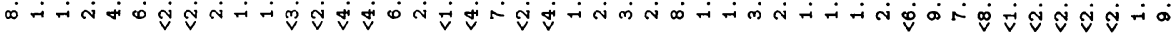

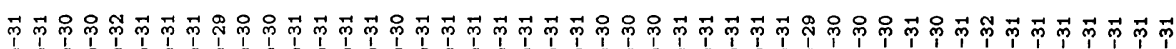

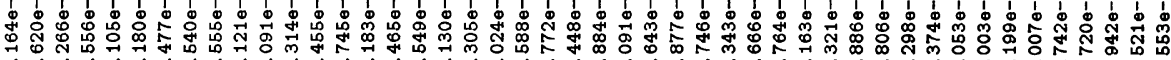

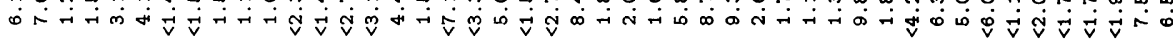

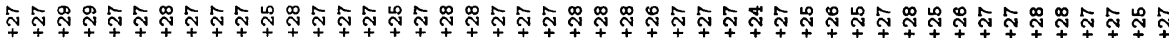

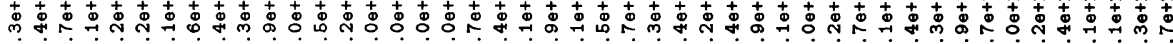

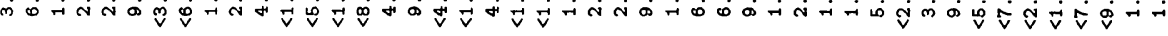

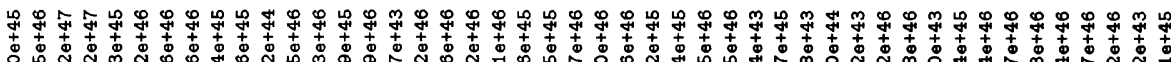
क नं कि

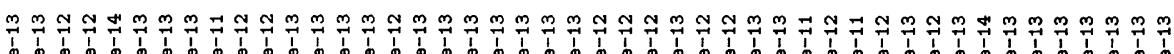
b

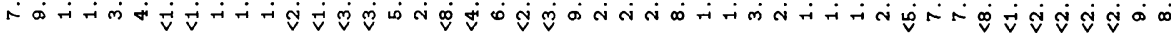

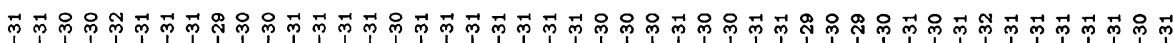

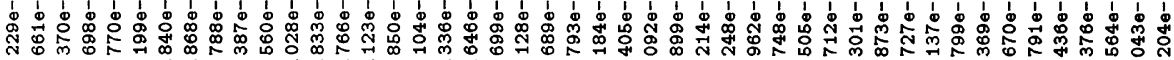

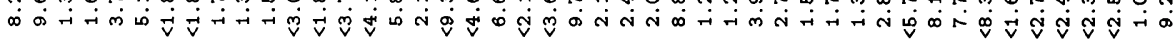

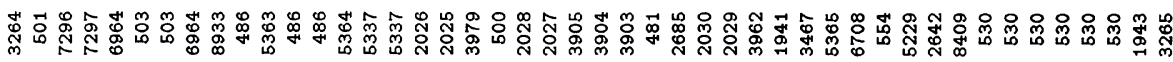

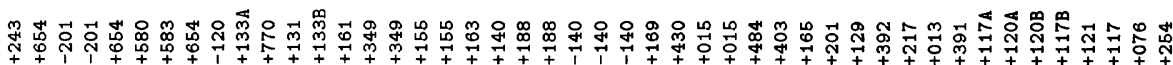

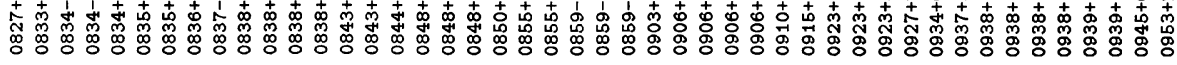




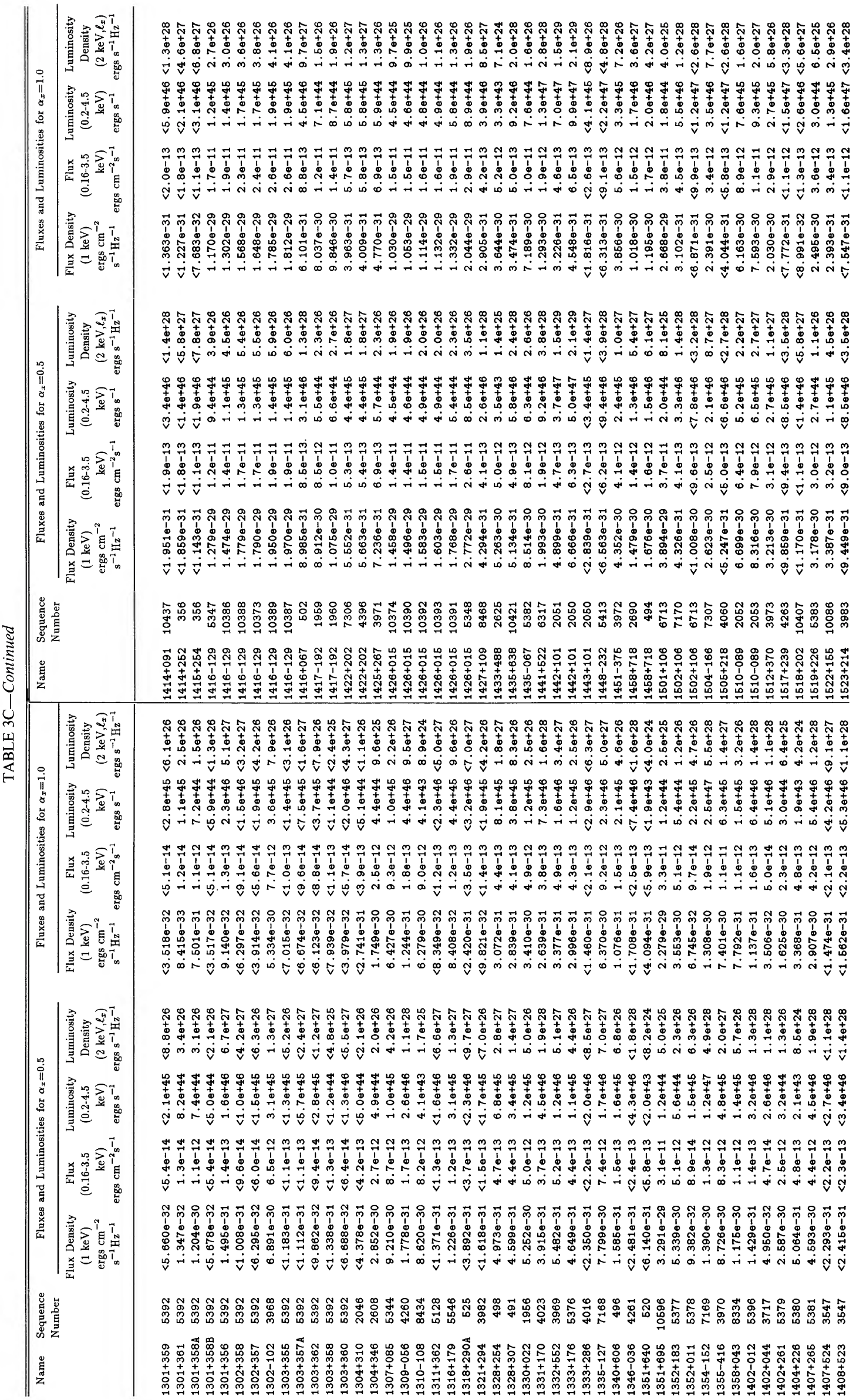


ํำ

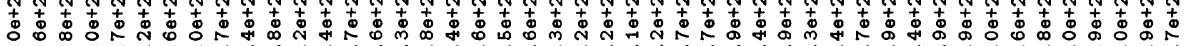

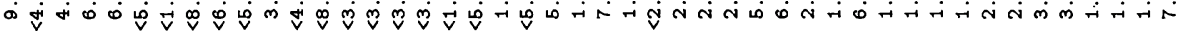

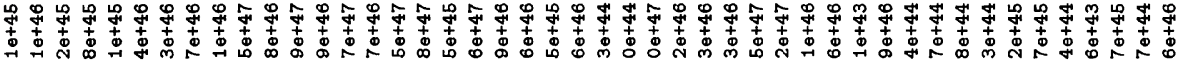

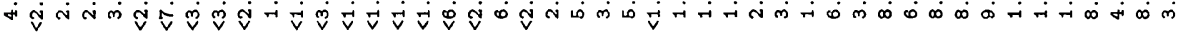

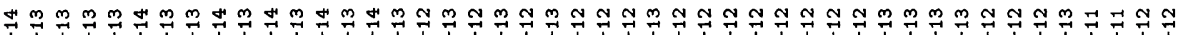

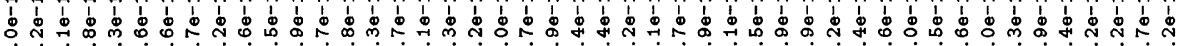

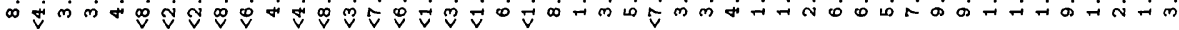

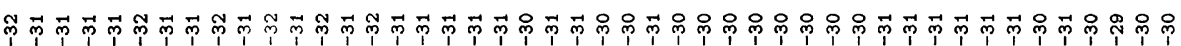

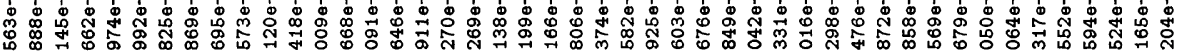
ம đูं

소

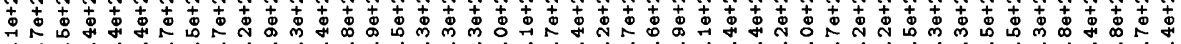
-

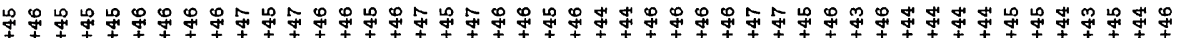

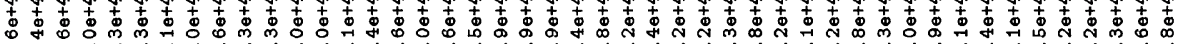

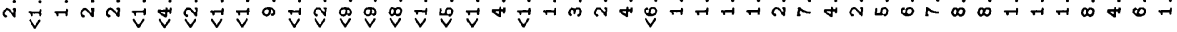

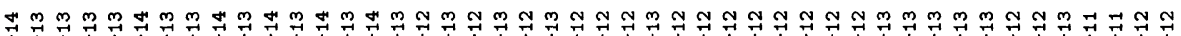

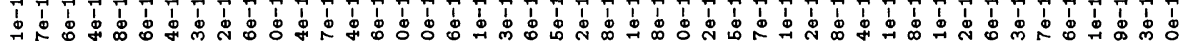
ヘ

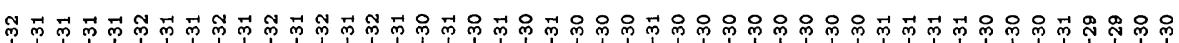

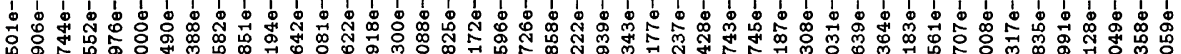
ヘ

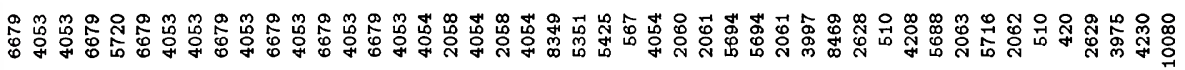

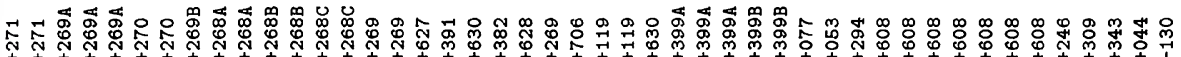

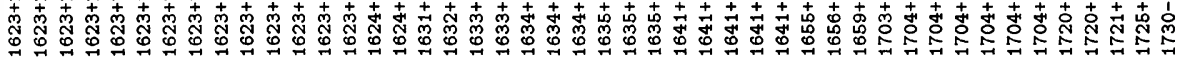

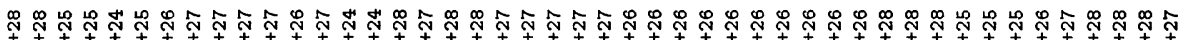

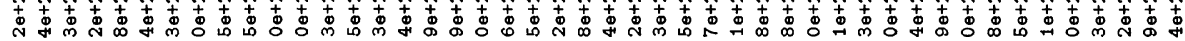

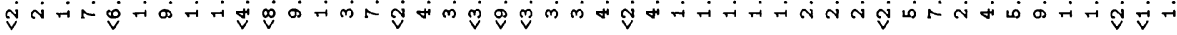

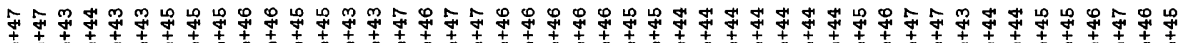

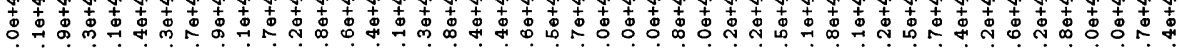

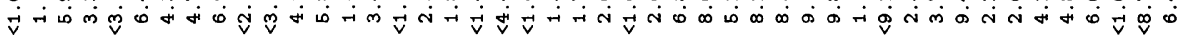

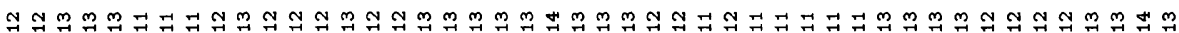

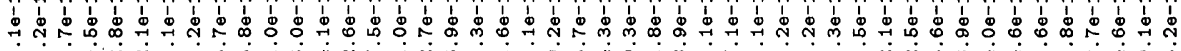
चेनितुल

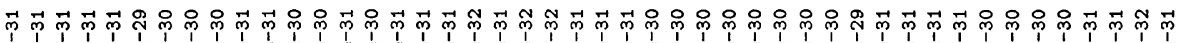

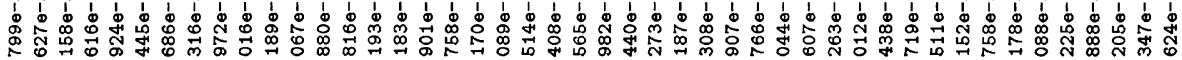

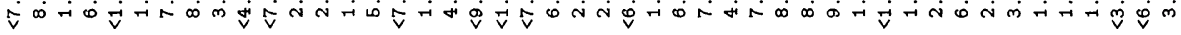

雨

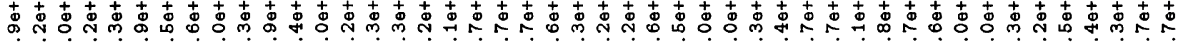
نं

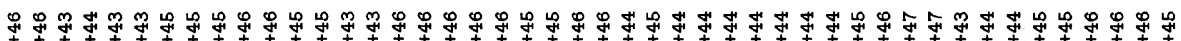

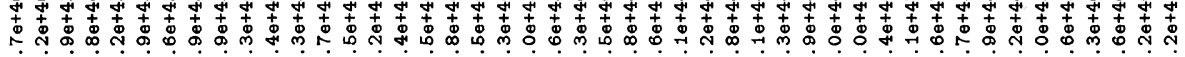

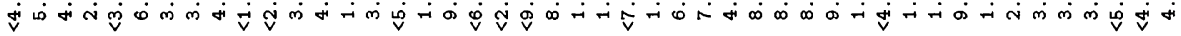

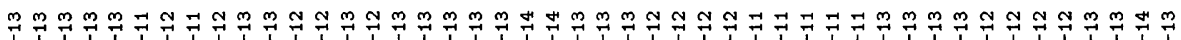

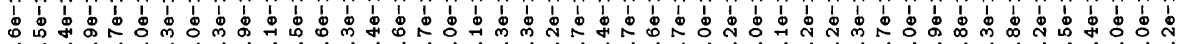

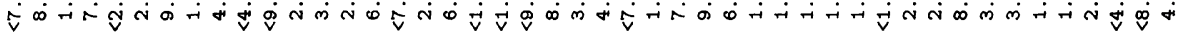
$\vec{p} \vec{p} \vec{p} \vec{p} \vec{p}$ 舟品

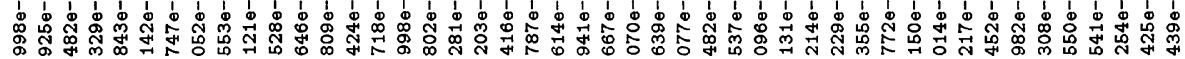
रें

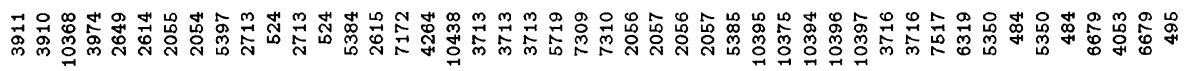

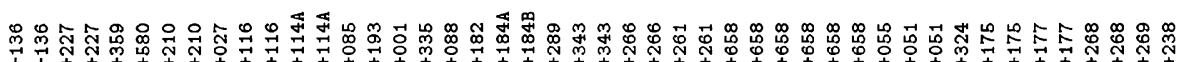

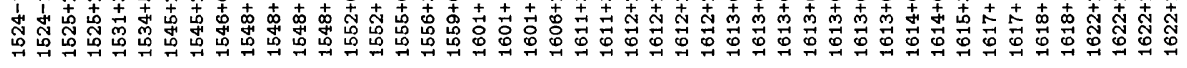




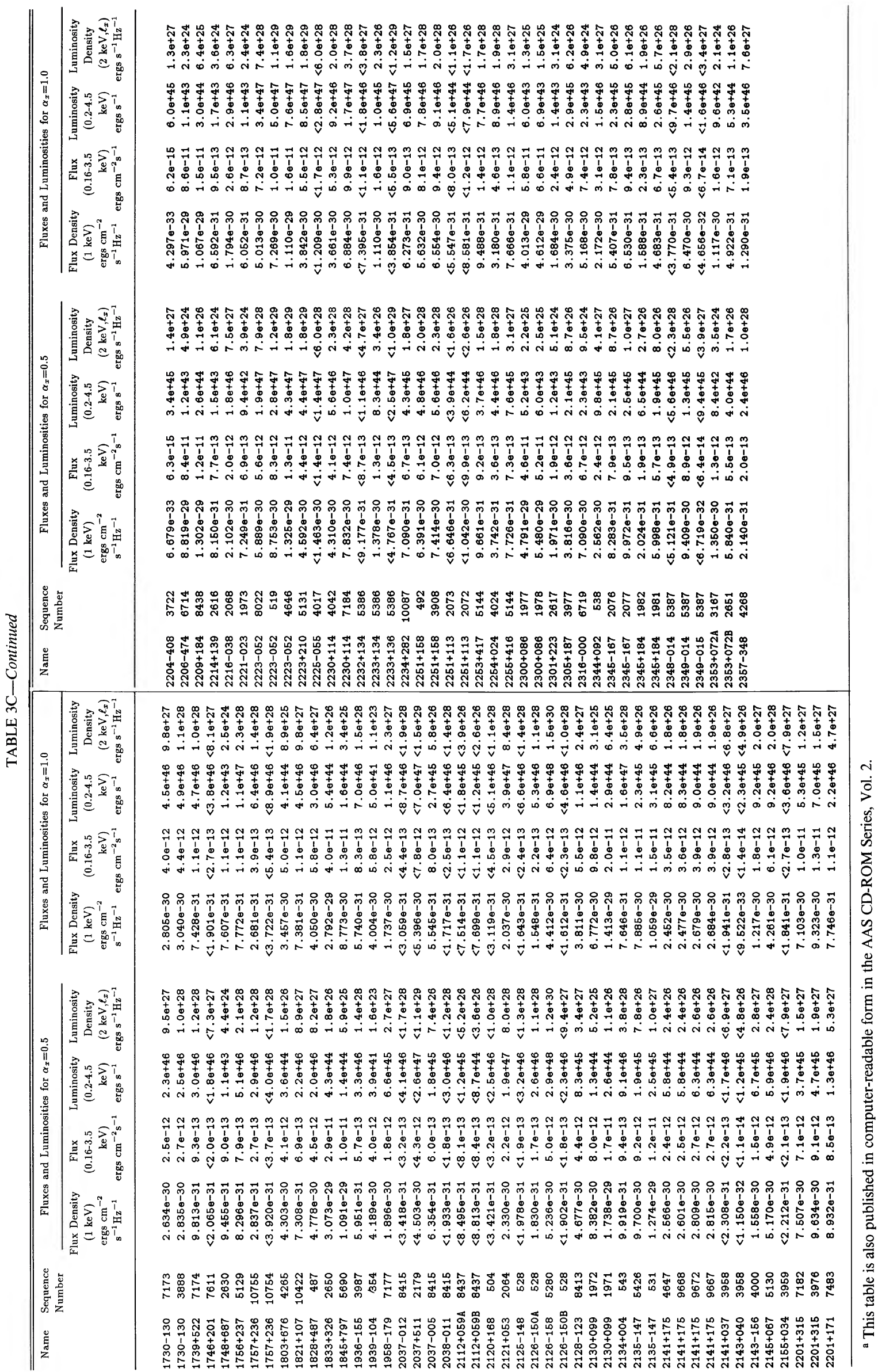




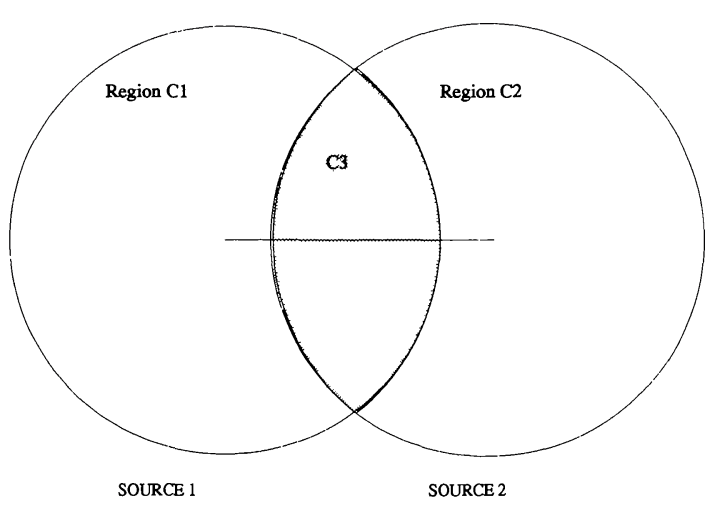

FIG. 1.-Schematic diagram showing the respective regions of overlapping source circles from which counts $C_{1}, C_{2}$, and $C_{3}$ are extracted.

increase the contaminating counts. The fraction of counts, $x$, from one source falling inside the $3^{\prime}$ circle around a nearby source, varies with source separation and with the pulse-height (PH) distribution of the counts, which in turn depends on the detector gain and the source spectrum. Since these sources are contaminating one another, we do not know the exact $\mathrm{PH}$ distribution for either source which limits the accuracy of our corrections for this effect. To quantify this situation, we examined the fraction of counts falling into a circle around a putative nearby source as a function of separation distance using seven observations of bright Seyfert galaxies and quasars covering a full range of the IPC gain: 12-18. For the source separations of interest $\left(2^{\prime}-4^{\prime}\right)$, the correction factor, $x$, was mostly dependent on the detector gain with separation a secondary factor. Mean values appropriate for the different gain ranges are shown in Table 4; we note that these values of $x$ are quite comparable to the scattering correction factor of $1.8 \%$ for isolated sources quoted in the previous section. Given the detector gain for the observation of interest, a correction based on the first results for $S_{1}$ and $S_{2}$ was computed and applied to $N_{1}$ and $N_{2}$. Values for $S_{1}, S_{2}$, and $\sigma_{1}$ were then recomputed.

This iterative process was repeated for five cycles which was usually more than sufficient for convergence. On a few occasions unphysical conditions (such as negative count rates) were encountered. We then decreased $x$ to $x-0.005$ under the assumption that the source spectrum is flatter than average, and restarted the iteration using the original net counts. For estimates of $S_{1}$ less than 0 , no iterative correction was applied since any contamination effect is insufficient to produce a positive net count rate for the target.

In the case of $N$ 's or $S$ 's being negative and $\left|N_{1}\right| \simeq\left|N_{2}\right|$, the estimate of $\sigma_{1}$ can become unstable due to a small denominator. To guard against unreasonably high error estimates, the error appropriate for the noncontaminated case, $\sqrt{C_{1}+C_{3}}$, was substituted if its value was lower than $\sigma_{1}$.

\subsection{Deep Survey Fields}

A background map for each observation is generated during the standard Rev1B processing by combining (1) the instrument flat field (DSMAP) generated from source-subtracted deep survey fields, which accounts for cosmic-ray background and non-uniformities in the detector; and (2) the diffuse back- ground (BEMAP) generated from data taken while looking at the bright Earth, which includes contributions from the sky and from solar-scattered X-rays. The contribution of the DSMAP is determined by the exposure time and that of the BEMAP from the source-subtracted background count rate in the image. In the standard processing, a first pass is made through the image using a local background estimate to detect and subtract sources in order to determine the background rate and evaluate the BEMAP contribution. A second source detection pass is then made using the combined DSMAP and BEMAP to find fainter sources. The final source list includes sources detected in both passes. For standard processing no subsequent correction is made to the background map to correct for any additional sources found in the second pass. While such a correction would be negligible in most cases, long observations may contain a number of weak sources which are not initially detected. This would result in an overestimate of the background rate and thus the level of the background map. We developed an iterative procedure to regenerate the background map after the second source detection search, repeat the search, and generate a final background map. This procedure was applied to fields with $>20000$ s of exposure time with the exception of those containing obvious diffuse sources. In these fields, no second source detection pass is made due to the large uncertainties involved, which in turn may lead to an overestimate of the background level and the failure to detect a relatively weak point source. The change in background level was significant only for the BF fields ( sequence numbers 5390 , $5391,5392)$, which contain an unusually large number of weak sources.

\subsection{Partially Obscured Objects}

There are a number of cases where the $3^{\prime}$ circle around a quasar position partially overlaps the IPC ribs which obscure $\mathrm{X}$-rays from a source, or where the circle is partially off the edge of the detector. For these, detection was determined from the counts available in a smaller box so the detection sensitivity is reduced. The source counts were measured from an unobscured half- or three-quarter circle and corrected for the fraction of area not used. If more than half the circle was obscured more than $5 \%$ of the time (due to small motions of the satellite), then the source was not processed and is listed as obscured or on the field edge in Table 5. Otherwise, the source counts were used to estimate the flux or upper limit in the usual way. The obscuration seriously decreases the area over which PH counts can be obtained from the image and in the case of the ribs presents an additional energy-dependent effect,

TABLE 4

\begin{tabular}{cc}
$\begin{array}{c}\text { CORRECTION FACTOR, } x, \text { AS A FUNCTION } \\
\text { OF DETECTOR GAIN }\end{array}$ \\
\hline \hline Gain & $\begin{array}{c}\text { Correction } \\
\text { Factor, } x\end{array}$ \\
\hline$\leq 14.0 \ldots \ldots \ldots \ldots \ldots \ldots$ & 0.025 \\
$14.0<$ Gain $\leq 15.0 \ldots \ldots \ldots$ & 0.020 \\
$>15.0 \ldots \ldots \ldots \ldots \ldots \ldots$ & 0.015 \\
\hline
\end{tabular}


preventing accurate determination of a PSF correction. A mean PSF correction of $1.8 \%$ (see $\S 3.1$ ) was applied for the partially obscured objects. Quasars processed in this way are noted in Table 2 (notes 2 and 3 ).

\subsection{Quasars Not Processed}

A number of objects could not be processed for a variety of reasons including multiple nearby sources, excessive obscura- tion by ribs/edge, mispointing, no aspect solution (useful information may still be obtainable for these objects), or unresolved computer processing errors (which may eventually be resolved). For completeness, they are listed in Table 5 along with the reason for their omission. For the user's convenience we also list omitted objects misclassified as quasars in the Einstein Observatory Catalog of Observations (5th edition), objects with no redshift and thus questionable quasars; a set of

TABLE 5A

OBJECTS NOT INCLUDED IN THIS PAPER

\begin{tabular}{|c|c|c|c|c|c|}
\hline Name & $\begin{array}{l}\text { Sequence } \\
\text { Number }\end{array}$ & Explanation & Name & $\begin{array}{l}\text { Sequence } \\
\text { Number }\end{array}$ & Explanation \\
\hline \multirow{2}{*}{$\begin{array}{l}0002+51 \ldots \ldots \cdots \\
0008-22 \ldots \ldots \ldots\end{array}$} & 8701 & No aspect $(\sim 1000 \mathrm{~s}$ of exposure $)$ & \multirow{2}{*}{$0920+39 \ldots \ldots \ldots$} & \multirow[t]{2}{*}{6738} & \multirow{2}{*}{$\begin{array}{l}\text { Classification unknown (search for } \\
\text { quasars) }\end{array}$} \\
\hline & 6727 & Classification unknown (search for & & & \\
\hline \multirow[t]{2}{*}{$0017+20 \ldots \ldots \ldots$} & \multirow[t]{2}{*}{6728} & \multirow{2}{*}{$\begin{array}{l}\text { quasars) } \\
\text { Classification unknown (search for } \\
\text { quasars) }\end{array}$} & $0931-11 \ldots \ldots \ldots$ & 6739 & \multirow{2}{*}{$\begin{array}{l}\text { Classification unknown (search for } \\
\text { quasars) } \\
\text { Unresolved error }\end{array}$} \\
\hline & & & $0938+119 \ldots \ldots$ & 530 & \\
\hline $\begin{array}{ll}0026+346 & \ldots \ldots \\
0057+311 & \ldots \ldots\end{array}$ & $\begin{array}{l}5141 \\
2619\end{array}$ & $\begin{array}{l}\text { No } z \\
\text { Obscured }\end{array}$ & $1107-11 \ldots \ldots \ldots$ & 6740 & $\begin{array}{l}\text { Classification unknown (search for } \\
\text { quasars) }\end{array}$ \\
\hline $0108+389 \ldots \ldots$ & 8464 & $\mathrm{X}$-ray-selected target & $1107+379$ & 3238 & Radio galaxy \\
\hline $0131-402 \ldots \ldots \ldots$ & 2578 & Obscured & \multirow[t]{2}{*}{$1138+041 \ldots \ldots$} & \multirow[t]{2}{*}{5356} & \multirow{2}{*}{$\begin{array}{l}\text { Nearby sources; target is a PG } \\
\text { quasar }\end{array}$} \\
\hline $0131-405 \ldots \ldots$. & 2578 & Unresolved error & & & \\
\hline $0131-404 \mathrm{~B} \ldots \ldots$ & 2578 & Unresolved error & \multirow{2}{*}{$1143-28 \ldots \ldots \ldots$} & \multirow[t]{2}{*}{6741} & \multirow{2}{*}{$\begin{array}{l}\text { Classification unknown (search for } \\
\text { quasars) }\end{array}$} \\
\hline $0131-406 \ldots \ldots$ & 2578 & Unresolved error & & & \\
\hline $0131-413 \ldots \ldots$. & 2578 & At field edge & $1215+303$ & 2715 & BL Lac \\
\hline $0132-403 \ldots \ldots$. & 2578 & Obscured & $1219+285$ & 2035 & BL Lac \\
\hline $0132-406 \ldots \ldots$. & 2578 & Incorrect optical position analyzed & $1227+074$ & 4052 & Obscured \\
\hline $0139+044 \ldots \ldots$. & 4057 & No $z$ & $1243-16 \ldots \ldots \ldots$ & 6742 & Classification unknown (search for \\
\hline $0143+077 \ldots \ldots$. & 4058 & No $z$ & \multirow{3}{*}{$\begin{array}{ll}1254+359 & \ldots \ldots \\
1254+362 & \ldots \ldots\end{array}$} & & quasars) \\
\hline \multirow[t]{2}{*}{$0207-14 \ldots \ldots \ldots$} & \multirow[t]{2}{*}{6729} & \multirow{2}{*}{$\begin{array}{l}\text { Classification unknown (search for } \\
\text { quasars) }\end{array}$} & & 5390 & Unresolved error (BF 30) \\
\hline & & & & 5390 & Obscured (BF 38) \\
\hline $0250-22$. & 6730 & $\begin{array}{l}\text { Classification unknown (search for } \\
\text { quasars) }\end{array}$ & $1254+59 \ldots \ldots \ldots$ & 2612 & $\begin{array}{l}\text { Mispointed (Mnk } 231 \text { intended } \\
\text { target at } 1254+37)\end{array}$ \\
\hline $0321-53$ & 4254 & Mispointed (intended target at & $1254+356 \mathrm{~B}$ & 5390 & Nearby sources (BF41) \\
\hline & & $0321-337)$ & $1300+362 \ldots$ & 5392 & Nearby sources (BF 202) \\
\hline $0323-24 \ldots \ldots \ldots$ & 6731 & $\begin{array}{l}\text { Classification unknown (search for } \\
\text { quasars) }\end{array}$ & $1302+361 \ldots \ldots$ & 5392 & $\begin{array}{l}\text { Nearby sources plus obscured (BF } \\
247 \text { ) }\end{array}$ \\
\hline $0327-24 \ldots$ & 6732 & $\begin{array}{l}\text { Classification unknown (search for } \\
\text { quasars) }\end{array}$ & $1303+31$ & 2046 & $\begin{array}{l}\text { Mispointed (B264 intended target } \\
\text { at } 1259+32)\end{array}$ \\
\hline $0332+07$ & 6733 & $\begin{array}{l}\text { Classification unknown (search for } \\
\text { quasars) }\end{array}$ & $1303+357 \mathrm{~B}$ & 5392 & $\begin{array}{l}\text { At edge of field of view requiring } \\
\text { unmasking (BF 275) }\end{array}$ \\
\hline $0348-04 \ldots$ & 6734 & Classification unknown (search for & $1318+290 \mathrm{~B}$ & $\begin{array}{r}525 \\
\end{array}$ & Analysis to be done (TON 156) \\
\hline & & quasars) & $1330+10 \ldots$ & 10352 & Unknown classification \\
\hline $0349-139 \ldots \ldots$ & 2346 & No $z$ & $1413+135 \ldots$ & 5143 & BL Lac \\
\hline $0350-09 \ldots \ldots \ldots$ & 2710 & $\begin{array}{l}\text { Mispointed (3C } 95 \text { intended target } \\
\text { at } 0349-14)\end{array}$ & $1430-15 \ldots \ldots \ldots$ & 6743 & $\begin{array}{l}\text { Classification unknown (search for } \\
\text { quasars) }\end{array}$ \\
\hline $0404-128 \ldots \ldots \ldots$ & 10648 & Unknown classification, no $z$ & $1505+220$ & 4060 & No $z$ \\
\hline $0458+13 \ldots \ldots \ldots$ & 6735 & Classification unknown (search for & $1515+231 \ldots \ldots \ldots$ & 8047 & No $z$ \\
\hline & & quasars) & $1519+257 \ldots \ldots$ & 4061 & No $z$ \\
\hline $0548-322 \ldots \ldots$. & 2707 & BL Lac & $1527+208$ & 4062 & No $z$ \\
\hline $0602+67 \ldots \ldots \ldots$ & 6736 & Classification unknown (search for & $1548+114 B$ & 524 & Nearby sources \\
\hline & & quasars) & $1642+690 \ldots$ & 7192 & No aspect ( $\sim 2200 \mathrm{~s}$ of exposure) \\
\hline $0627-19 \ldots$ & 6737 & Classification unknown (search for & $1749+701 \ldots \ldots$ & 2720 & BL Lac \\
\hline & & quasars) & $1928+738 \ldots \ldots$ & 7589 & No aspect ( $3000 \mathrm{~s}$ of exposure) \\
\hline $0731+653 \ldots \ldots$ & 5227 & Incorrect optical position analyzed & $2044-027 \ldots$ & 8981 & Short exposure $(\sim 300 \mathrm{~s})$; analysis \\
\hline $0809+04 \ldots \ldots \ldots$ & 2719 & Mispointed (unknown & & & to be done \\
\hline & & $\begin{array}{l}\text { classification for target at } \\
0829+47 \text { ) }\end{array}$ & $2047+09 \ldots$ & 6744 & $\begin{array}{l}\text { Classification unknown (search for } \\
\text { quasars) }\end{array}$ \\
\hline $0830+112 \ldots \ldots \ldots$ & 2023 & X-ray-selected target & $2117+02$. & 552 & BL Lac \\
\hline $0830+112 \ldots \ldots \ldots$ & 2024 & X-ray-selected target & $2201+044 \ldots \ldots$ & 553 & BL Lac \\
\hline $0844+186 \ldots \ldots$. & 4059 & No $z$ (target differs from X-ray- & $2251-178 \ldots \ldots$ & 2074 & X-ray-selected target \\
\hline & & selected QSO 5' away) & $2252-09 \ldots \ldots \ldots$ & 6746 & Classification unknown (search for \\
\hline $0855+14 \ldots \ldots \ldots$ & 8980 & No aspect (satellite probably & & & quasars) \\
\hline $0912+297 \ldots$ & 2716 & mispointed) & $2330+08$. & 6747 & $\begin{array}{l}\text { Classification unknown (search for } \\
\text { quasars) }\end{array}$ \\
\hline & 2710 & BL Lac & & & quasars) \\
\hline
\end{tabular}

NoTE.-See text for details.

${ }^{a}$ This table is also published in computer-readable form in the AAS CD-ROM Series, Vol. 2. 
TABLE 5B

OBJeCtS INCLUDED IN THIS PAPER, WITH INCOMPLETE ANALYSIS FOR INDIVIDUAL OBSERVATIONS LISTED HERE ${ }^{\mathrm{a}}$

\begin{tabular}{|c|c|c|}
\hline Name & $\begin{array}{l}\text { Sequence } \\
\text { Number }\end{array}$ & Explanation \\
\hline $0121-590$. & 6726 & No aspect $(\sim 1800 \mathrm{~s}$ of exposure $)$ \\
\hline $1258+356$. & 5391 & Unresolved error (BF 141) \\
\hline $1259+357$. & 5391 & Obscured (BF 161) \\
\hline $1259+361$. & 5391 & Obscured (BF 164) \\
\hline $1259+360$. & 5391 & Obscured (BF 170) \\
\hline $1622+268$ & 5720 & Analysis to be done (KP 70) \\
\hline $1622+269$. & 4053 & Obscured (KP 71) \\
\hline $1622+269$. & 5720 & Analysis to be done (KP 71) \\
\hline $1623+271 \ldots$. & 5720 & Analysis to be done (KP 72) \\
\hline $1623+270 \ldots \ldots$ & 5720 & Analysis to be done (KP 73) \\
\hline $1623+269 B$ & 6679 & Nearby sources (KP 74) \\
\hline $1623+269 B$ & 5720 & Analysis to be done (KP 74) \\
\hline $1623+268 \mathrm{~A}$. & 5720 & Analysis to be done (KP 76) \\
\hline $1623+268 B$ & 5720 & Analysis to be done (KP 77) \\
\hline $1623+268 \mathrm{C}$ & 5720 & Analysis to be done (KP 78) \\
\hline $1624+269 \ldots$ & 5720 & Analysis to be done (KP 79) \\
\hline $2141+175 \ldots$ & 9669 & No aspect (satellite mispointed) \\
\hline $2141+175 \ldots \ldots$. & 9670 & No aspect (satellite mispointed) \\
\hline
\end{tabular}

${ }^{a}$ This table is also published in computer-readable form in the AAS CD-ROM Series, Vol. 2.

objects listed as quasars in the Yellow Book (Seq. 6727-6747) which in reality were targets hypothesized, but not confirmed, as quasars with possible high X-ray luminosity, and a few objects not yet properly processed due to coordinate errors. Table $5 \mathrm{~A}$ lists such objects which have been omitted from this paper, while Table 5B is comprised of objects for which results are reported here but for which one or more observation sets (sequences) have not yet been fully analyzed.

\subsection{Optical Data}

Optical $B$ and / or $V$ magnitudes, collated primarily from the quasar catalogues (Hewitt \& Burbidge 1987, 1989, hereafter HB87 and HB89; Véron-Cetty \& Véron 1987, hereafter VV87), are listed in Table 6 along with the reference. For these two catalogs, magnitudes of quasars without color data are quoted as " $V$." In many cases the original references provide additional information (such as photographic magnitude which approximates $B$ ). To the extent possible, we rechecked the literature for objects without color information in the catalogues. Where applicable, we changed " $V$ " magnitudes in the catalogs to $B$ in Table 6 and indicated such objects by a 2 in the Note column. Additional reference information is provided by other entries to the Note column of Table 6 for several quasars for which the catalog magnitudes have been superseded. In some cases, such as the PG quasars, we used the original reference (e.g., Schmidt \& Green 1983) and $B$ magnitudes, which occasionally differ slightly from the values in HB87 and HB89, and VV87. When $V$ magnitudes from the quasar catalogs for PG quasars are included in Table 6, the $B-V$ color does not always agree with the color difference quoted in the quasar catalogs. For other samples, such as Sramek \& Weedman (1980) and Anderson (1990), the available magnitudes are essentially $B$ and are quoted as such in Table 6 without an accompanying note, even though HB87 and HB89 and VV87 may list the same data as $V$ magnitudes. We calculated the $2500 \AA$ (rest frame) optical luminosity, $l_{o}$, from the $B$ magni- tude, where available, assuming a power-law energy distribution with slope $\left(\alpha_{o}\right)$ of 0.5 . This calculation was made using a magnitude-to-flux conversion constant of 48.36 for $B$ magnitudes (Hayes \& Latham 1975) leading to the following equation for the emitted flux at $2500 \AA$ :

$$
\begin{aligned}
& \log \left[f_{\mathrm{em}}(2500)\right]=-19.34+\alpha_{0} \log \left(\frac{2500}{4400}\right) \\
& -\left(1-\alpha_{0}\right) \log [1+z]-0.4\left(B-A_{B}+\Delta B\right) .
\end{aligned}
$$

When no $B$ magnitude was available, the $V$ magnitude was used with a conversion constant of 48.60 (Oke 1974) leading to a constant of -19.44 in the equation above.

When no $B$ magnitude was available, the $V$ magnitude was used with a conversion constant of 48.60 (Oke 1974) leading to a constant of -19.44 in the equation above.

The correction for reddening along the line of sight through our Galaxy assumes the constant gas-to-dust ratio given by Burstein \& Heiles (1978) so that

$$
E_{B-V}=\max \left[0,\left(-0.055+1.987 \times 10^{-22} N_{\mathrm{H}}\right)\right],
$$

where $N_{\mathrm{H}}$ is the Galactic value as used to determine the X-ray luminosity. Following Allen (1973), we then take

$$
\begin{gathered}
A_{V}=3 E_{B-V} \\
A_{B}=A_{V}+E_{B-V}=4 E_{B-V} .
\end{gathered}
$$

The correction for the presence of emission lines in the $B, V$ filter range uses the formula

$$
\Delta B=2.5 \log _{10}\left[1+W_{\lambda}(1+z) \frac{R_{\mathrm{B}}(\lambda)}{\int R_{B}(\lambda) d \lambda}\right]
$$

where $W_{\lambda}$ is the rest frame equivalent width in $\AA$ of the emission line; $\lambda=\lambda_{\text {rest }}(1+z)$, is the observed wavelength of the line at redshift $z$; and $R_{B}$ is the response of the $B$ filter in $\AA^{-1}$ (and similarly for $V$; Marshall 1983). Adopted mean equivalent widths for prominent emission lines were taken from Wilkes (1986) and are shown in Table 7. No correction was made for the contribution of starlight which is expected to be important at low $l_{o}\left(\log l_{o} \leq 29\right)$. This does effect the results of our analysis ( see $\S 5.3$ ).

We note that the values of $\log l_{o}$ (the spectral luminosity at $2500 \AA$ in units of $\mathrm{erg} \mathrm{s}^{-1} \mathrm{~Hz}^{-1}$ computed using eq. [6] assuming $\alpha_{o}=0.5$ ) are lower than those reported in earlier papers (Marshall et al. 1984; AT86; Worrall et al. 1987) by 0.06 on average. This is due to a combination of updated constants for the magnitude-to-flux conversion, a more accurate reddening correction, and generally smaller corrections for emission lines due to updated equivalent width measurements. This mean change results in a negligible change in the resulting $\alpha_{o x}$ values of 0.002 .

The effective optical-to-X-ray power-law slope, $\alpha_{o x}$, also listed in Table 6, was computed following the definition:

$$
\alpha_{o x}=-\frac{\log \left(l_{x} / l_{0}\right)}{\log \left(\nu_{x} / \nu_{\mathrm{opt}}\right)},
$$




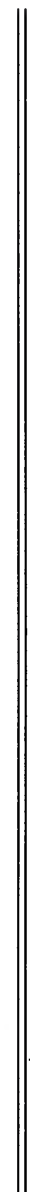

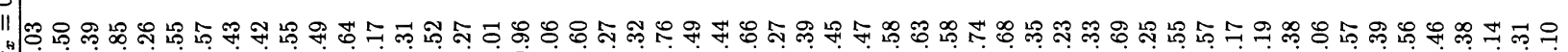

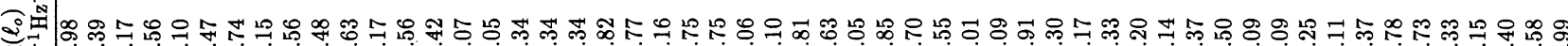
员

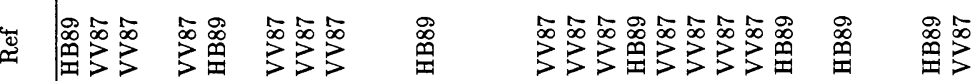

\begin{tabular}{|c|c|c|c|c|c|c|c|c|}
\hline 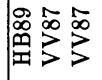 & $\stackrel{\infty}{\infty}^{\infty} \stackrel{\infty}{\stackrel{\infty}{M}}$ & $\sum_{>}^{\infty} \sum^{\infty} \sum^{\infty}$ & 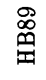 & 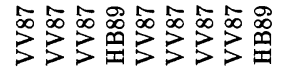 & 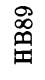 & 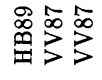 & 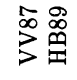 & 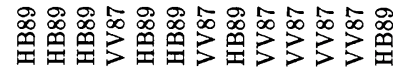 \\
\hline 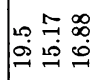 & 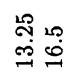 & 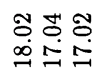 & 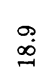 & 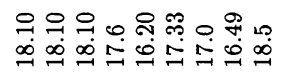 & $\stackrel{\oplus}{\oplus}$ & 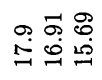 & 苟苛 & 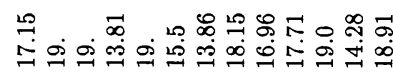 \\
\hline
\end{tabular}

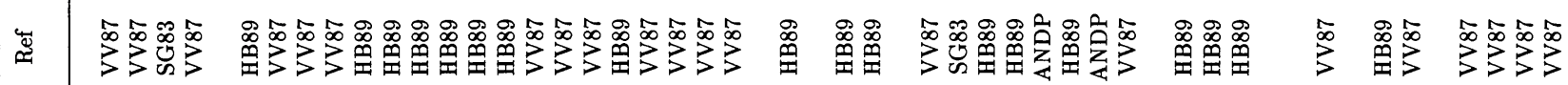

z

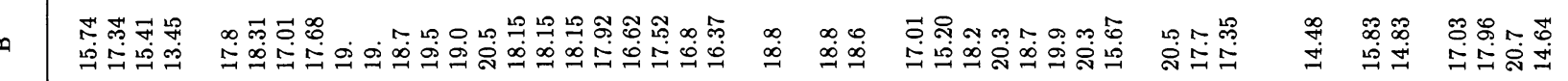

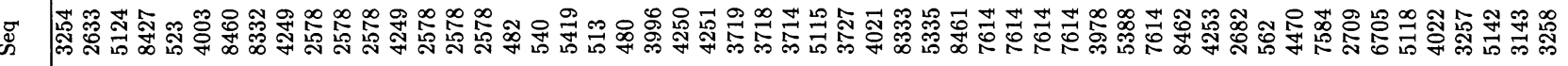

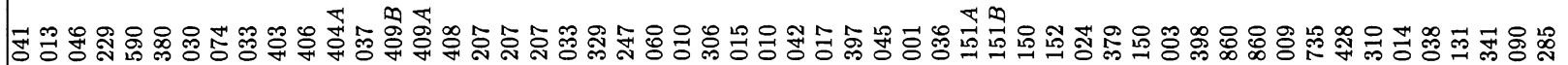

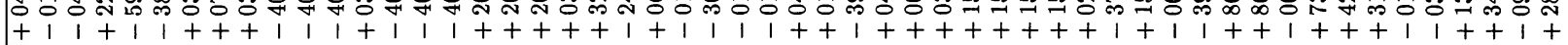

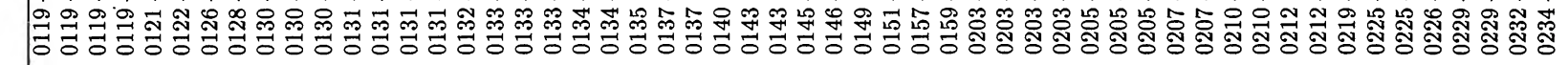

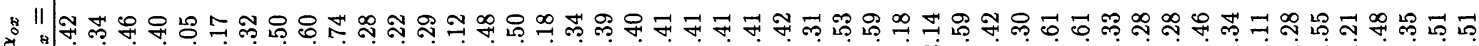
ठै

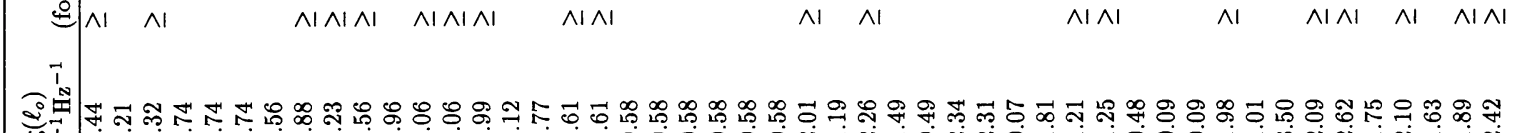

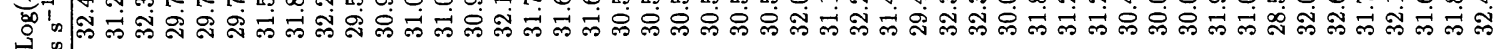
象

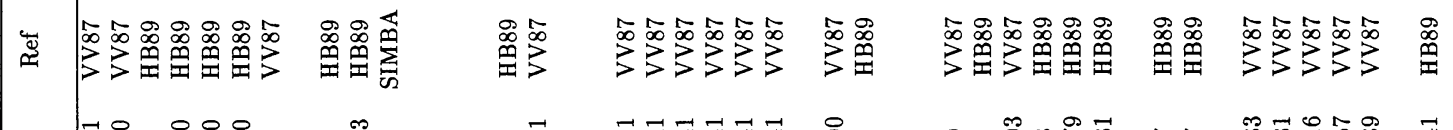

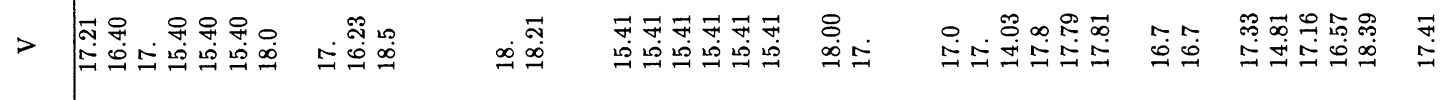

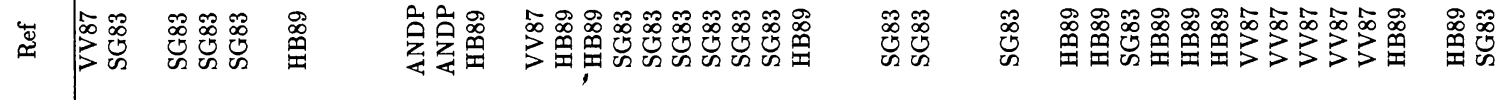

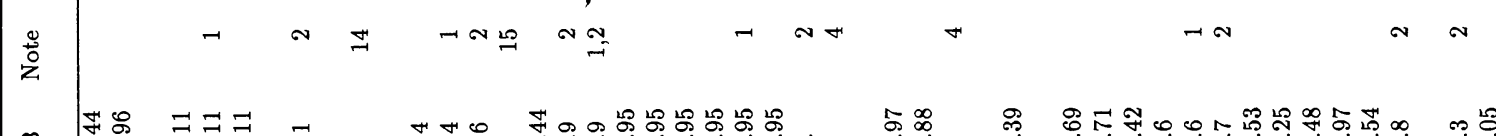

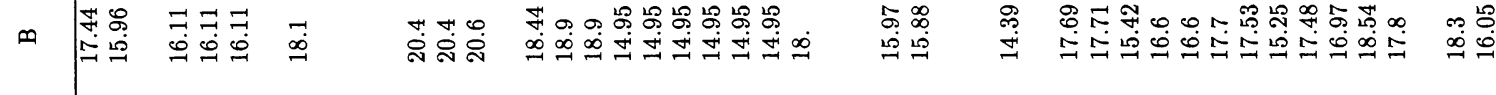

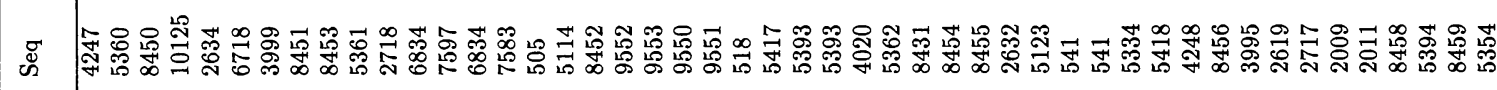
ฟึ - $1+1++++11+++++++++++++++11++++++++++++++1+++++1,1+t$

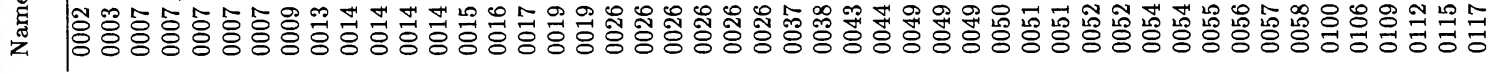


ไ||

.

$\wedge \mathrm{A} \mathrm{I}$

$\wedge I \wedge I \wedge I \wedge I N I N I N I \wedge I$

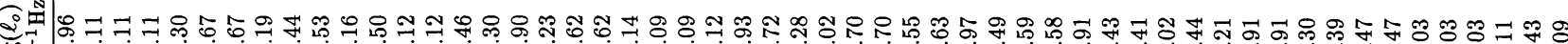

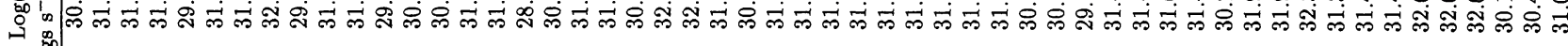

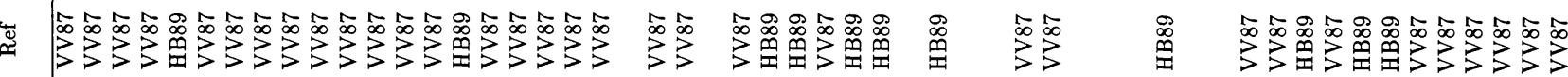

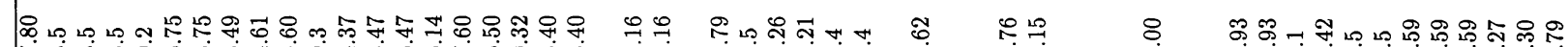
영

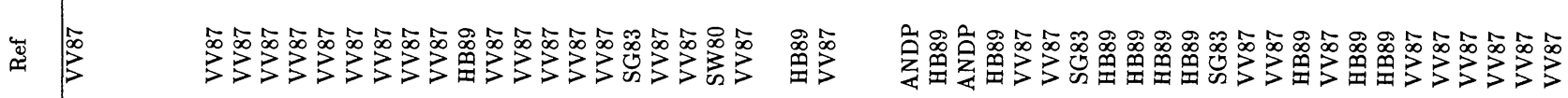

$\stackrel{ \pm}{\stackrel{2}{z}}$

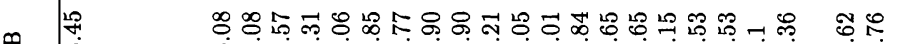

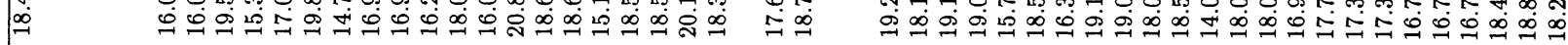

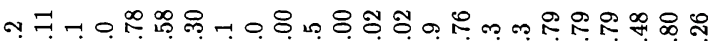

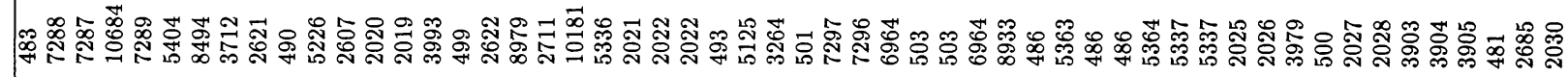

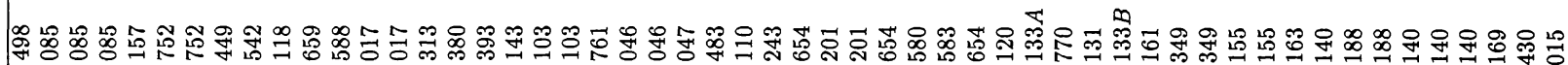
$+1|1| 11+++++++++++++++++++++11++++1+++++++++++++111+++$

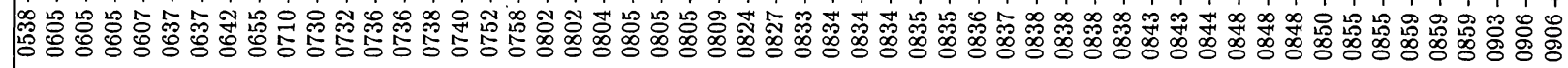

"11 $\wedge I \wedge I \wedge I \quad \wedge I \wedge I$

$\wedge \mathrm{\wedge}$

$\wedge \wedge \wedge$

$\wedge \mathrm{I}$

2

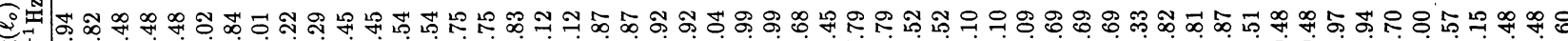

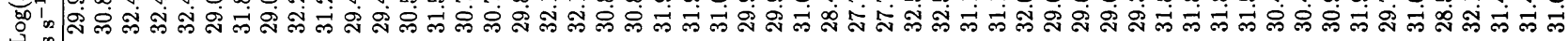
要

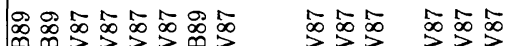

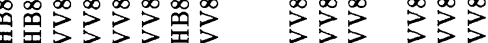

$>>>>3$

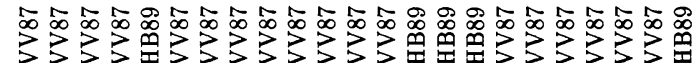

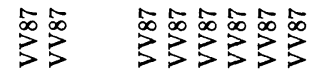

ㅇำ

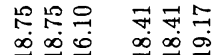

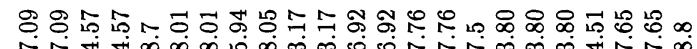

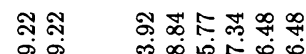

הi்

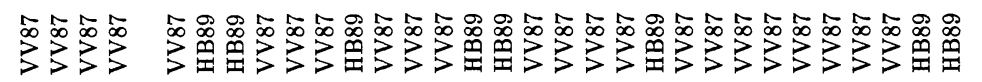

\begin{abstract}
N N -
\end{abstract}

\begin{abstract}
-
\end{abstract}
$\stackrel{20}{\rightarrow}$

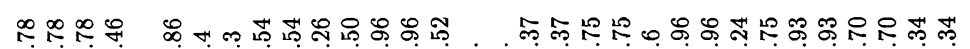

仓்

$\sum^{\infty} \sum^{\infty} \sum^{\infty} \sum^{\infty} \sum^{\circ}$

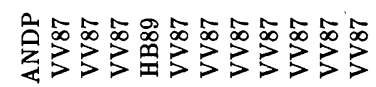

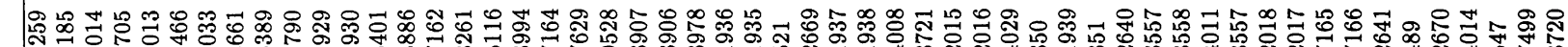

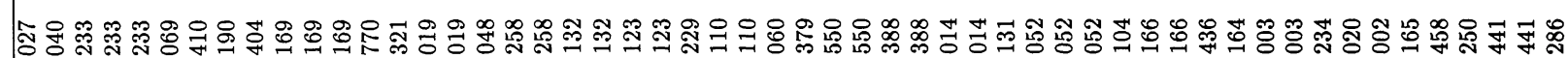
$1+111+1+1+++1+11+++1111+++1+11_{1} 11111+++1111111111+11111$

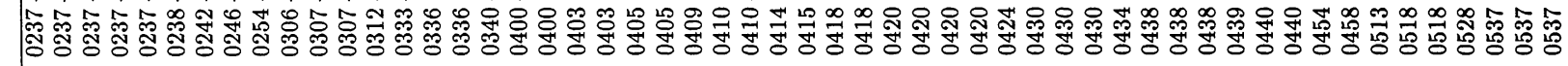


ل11
常 $\wedge 1 \wedge 1 \wedge 1 \wedge 1$
$\wedge I \wedge I \wedge I \quad \wedge I$
$\wedge I \wedge I \wedge I \quad \wedge I$
$\wedge I \wedge I \wedge I$

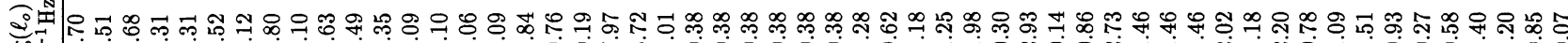

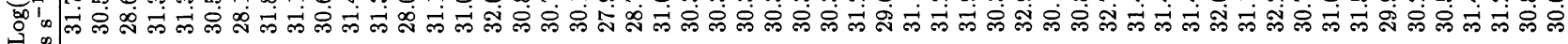

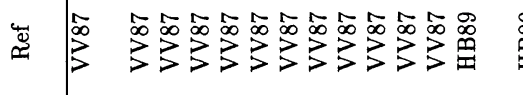

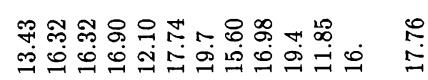

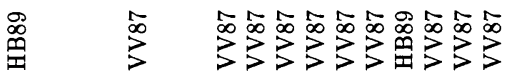

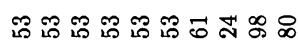

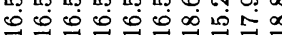

$\sum^{\infty} \sum^{\infty} \sum^{\infty} \sum^{\infty} \sum^{\infty} \sum^{\infty} \sum^{\infty} \stackrel{\infty}{\infty} \quad \sum^{\infty} \hat{\infty}$

ㄱ. $\sum_{\substack{\infty \\ \infty}}^{\infty}$

$\exists$

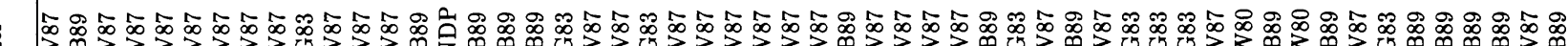

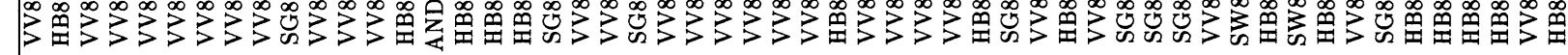

당요 웅

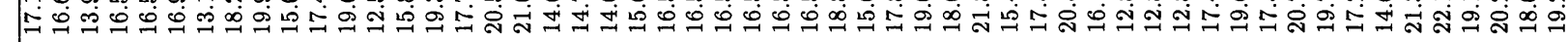

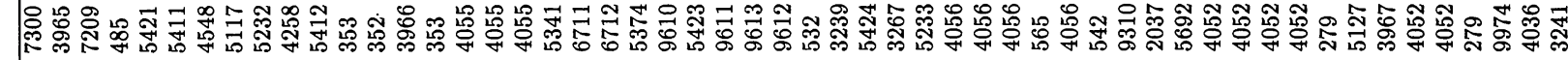

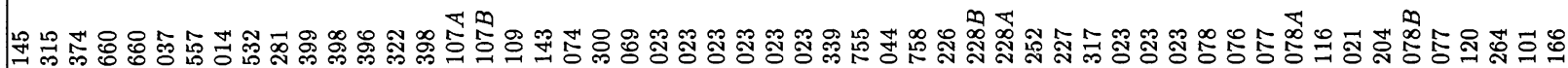

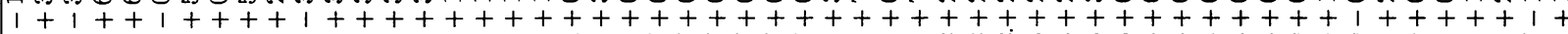

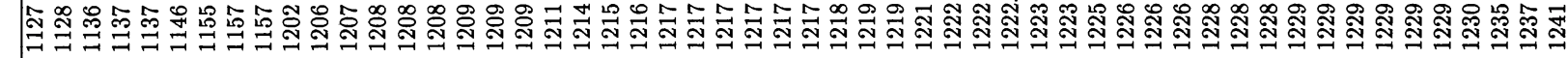

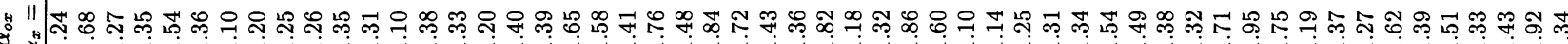
:$$
\wedge
$$
$\wedge I \wedge I \wedge I \wedge I \wedge I \wedge I$
$\wedge$
$\wedge I \wedge \mid \wedge I \wedge I$
$\wedge \mathrm{I} I$
$\wedge 1$
$\wedge|\wedge|$
$\wedge$

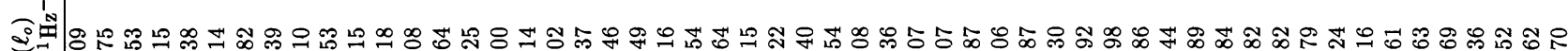
金的

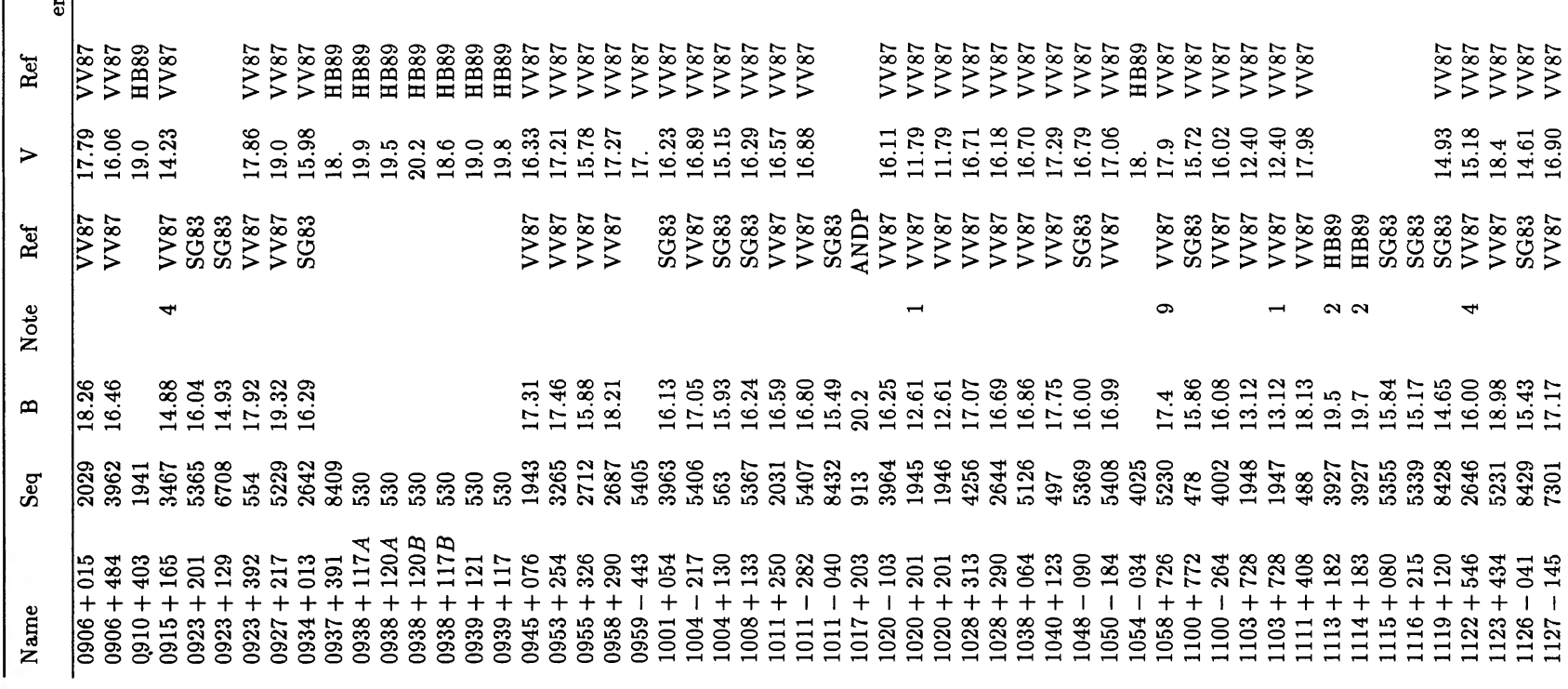


11

氛|

$\Lambda I N I N I N I N I N$

$\wedge I \wedge I$

$\wedge I \wedge I$

$\wedge I \wedge I \wedge I \wedge I N I$

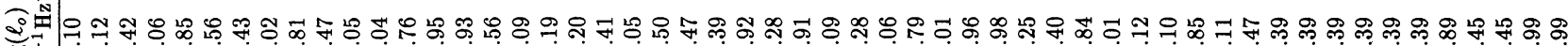
๒ cond

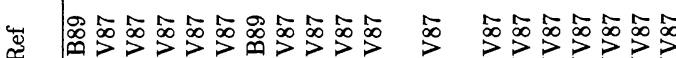

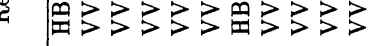

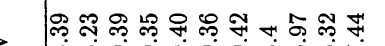

$3^{\infty} 3^{\infty} 3^{\infty} 3^{\infty} \sum^{\infty} \sum^{\infty} \sum^{\infty}$

$\stackrel{\infty}{D^{\infty}} \sum^{\infty} \sum^{\infty} \sum^{\infty} \sum^{\infty}$

$\sum^{\infty} \sum^{\infty} \sum^{\infty}$

$\sum^{\infty} \sum^{\infty} \sum^{\infty} \sum^{\infty} \sum^{\infty} \sum^{\infty} \sum^{\infty}$

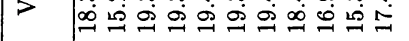

ఈ กิฒ

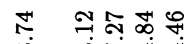

$\therefore \div$

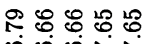

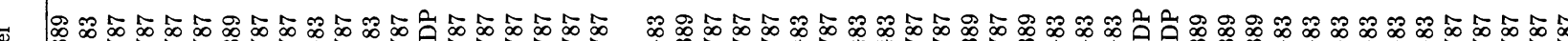

我

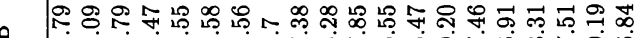

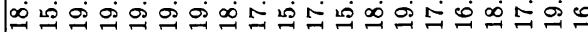

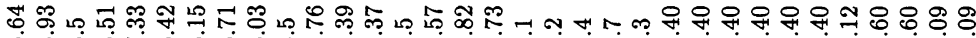

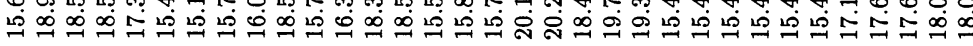

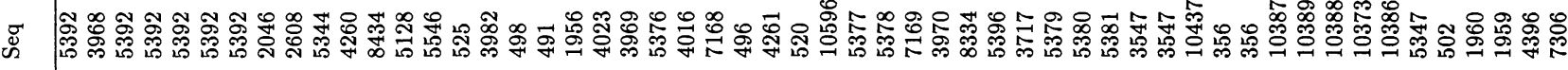

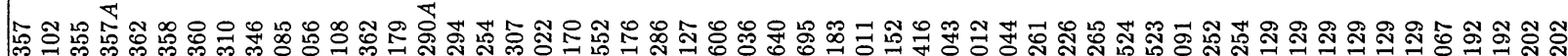

$+1++++++++11+++++++++++1+1++++11+1+++++++++1111111+11+t$

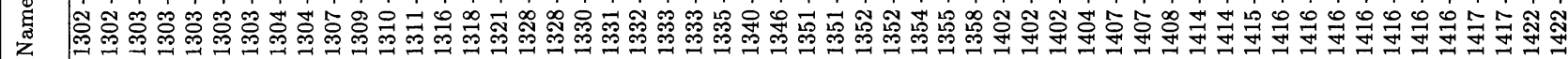

(2).

范

$\wedge I \wedge I \wedge I \wedge I \wedge I \wedge I \wedge I N I N$

$\wedge \mathrm{I}$

$\wedge I \wedge I N I \wedge I N I$

$\wedge I \wedge I \wedge I \wedge I \wedge I \wedge I$

$\wedge \mathrm{\wedge I}$

$A I N I \quad N I N I N I N I N I N I$

$\wedge \wedge \wedge$

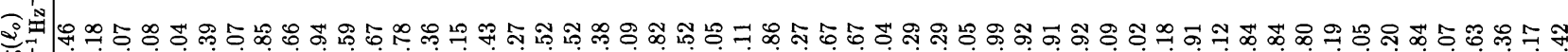

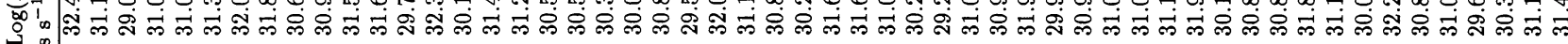

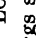

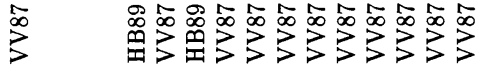

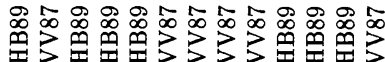

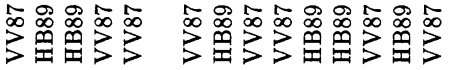

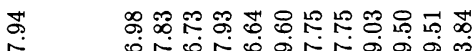

응 ศำ \&

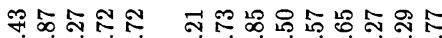

I

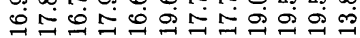

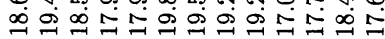

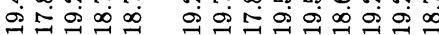

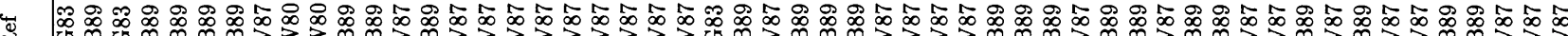
党
N N N N N
N
$\rightarrow$
$\bumpeq \stackrel{\infty}{\sim} \sim$
$\rightarrow \sim$
오요

苋

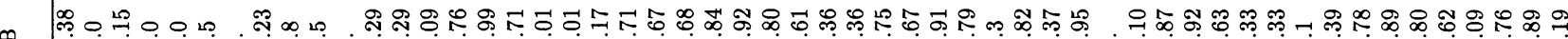

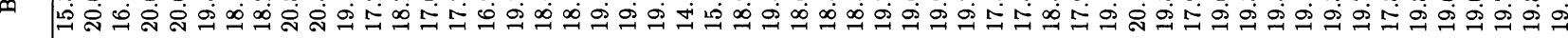

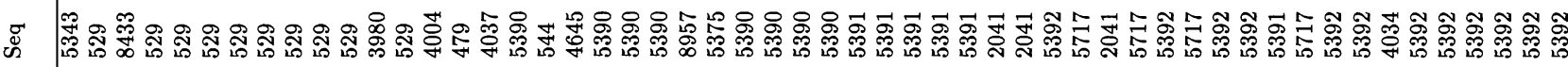

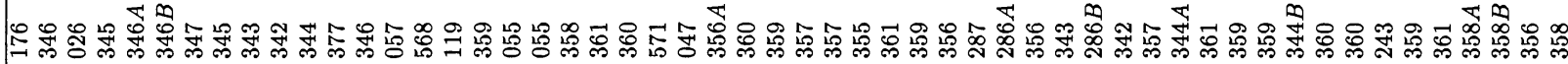

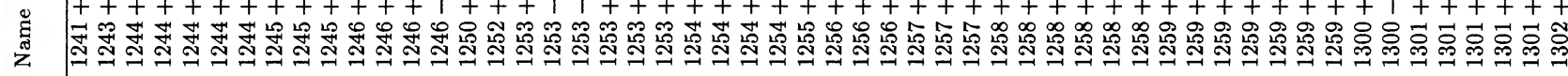




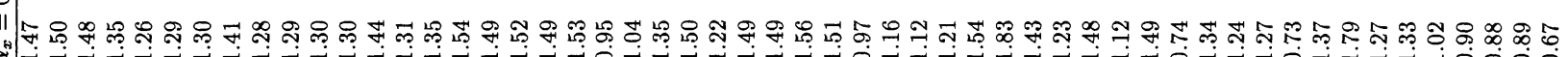

$\wedge$

$\wedge \mathrm{I} \wedge \mathrm{I} \wedge \mathrm{I}$

NININININI NINININININININI NI

$\wedge 1$

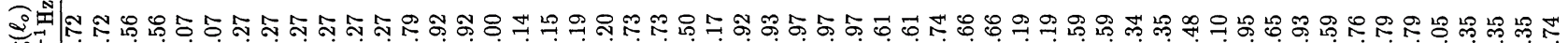

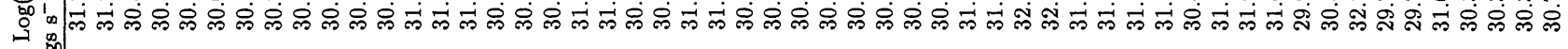

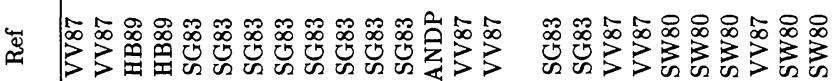

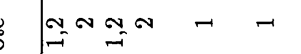

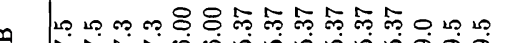

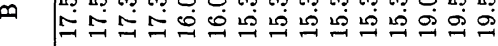

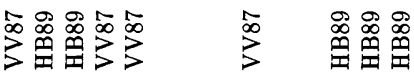

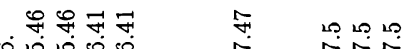

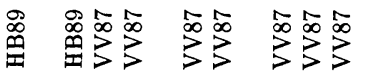

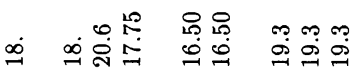

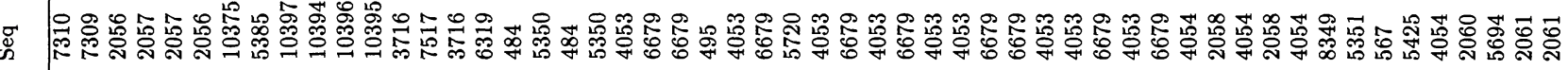

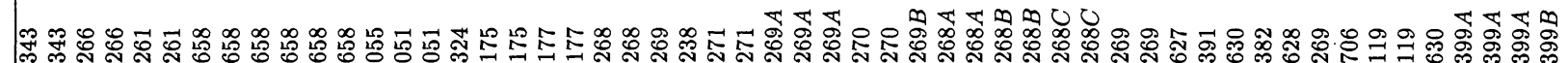

$+++++++++++++++++++++++++++++++++++++++++++7++++++$

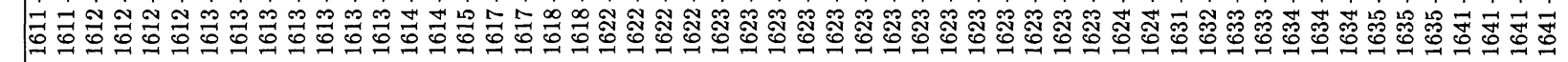

11 고 จ ని

$\wedge \perp \wedge \mathrm{I}$

$\wedge \wedge 1$

$\wedge 1$

$\wedge \mid \wedge !$

$\wedge I \quad \wedge I \wedge I \wedge I$

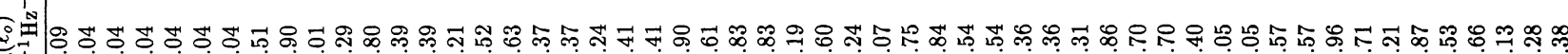
फ़

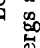

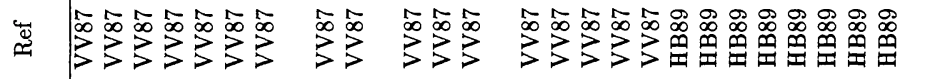

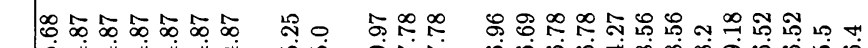

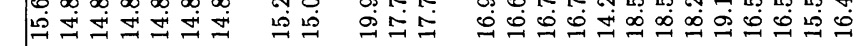

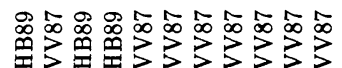

เค

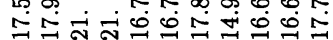

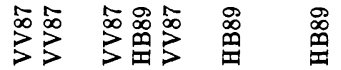

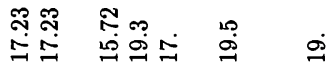

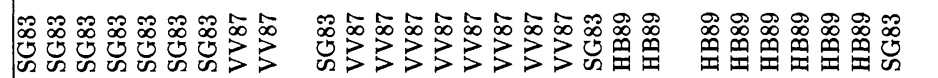

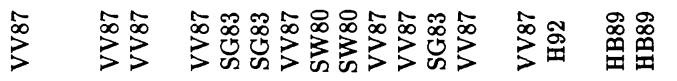

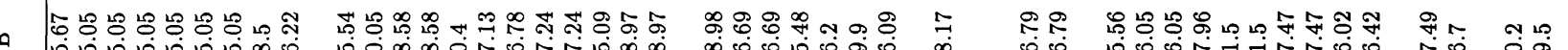
ه

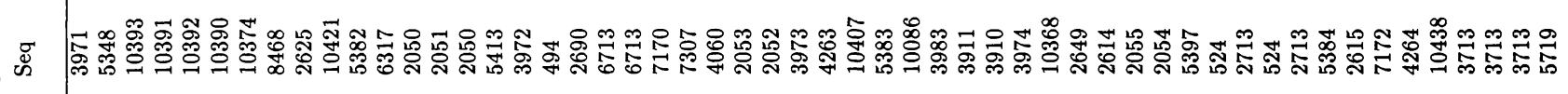

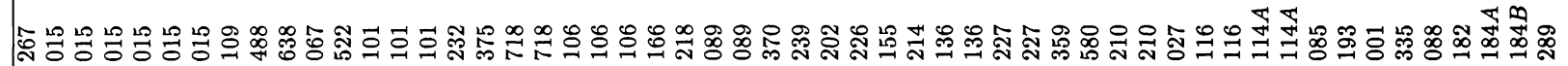
$+++++++++1++++11+++++1+11++++++11+++++++++++++++++++++$

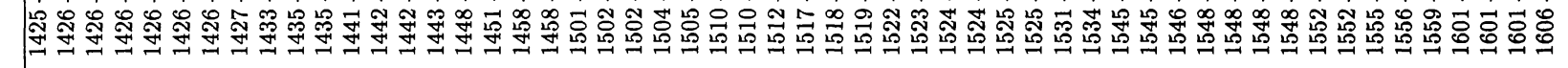




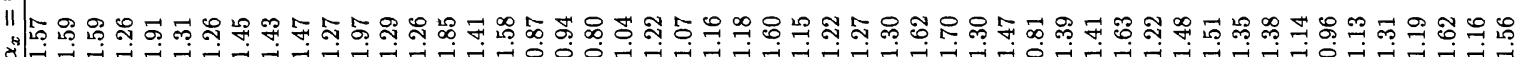
仓ी $\wedge 1 \wedge$
$\wedge \mathrm{I} \wedge \mathrm{I} \wedge \mathrm{I}$
$\wedge \mathrm{N} \wedge$
$\wedge \wedge \wedge$

领

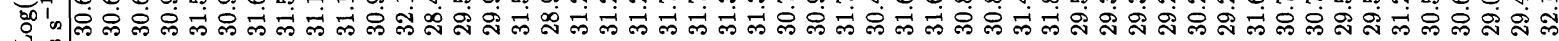
每

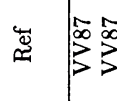

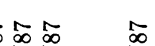

\section{-}

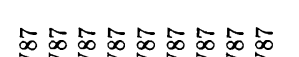

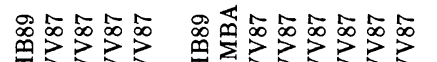

$\sum^{\infty} \sum^{\infty} \sum^{\infty} \sum^{\infty} \sum^{\infty} \sum^{\infty}$

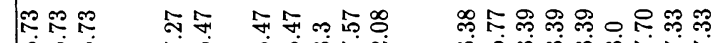

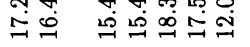

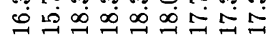

으응 요

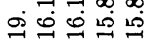

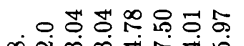

ஸீ:

$>\quad$ is 19

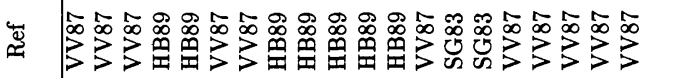

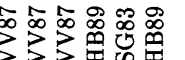

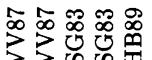

z

$\infty$

万எ

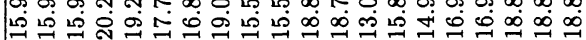

$\rightarrow \sim$

$-\rightarrow$

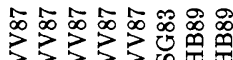

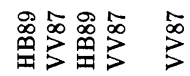

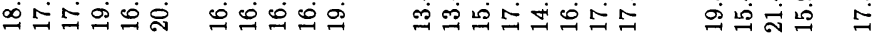

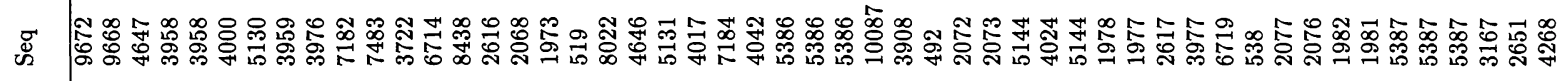

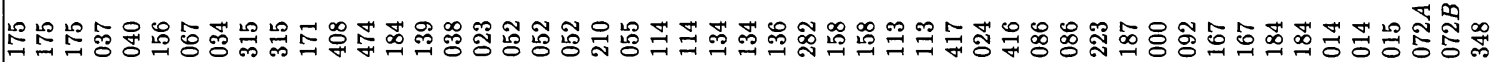

$+++++1+++++111++1|1| 1 \mid 1+1+++++++++++++++++1+11++111++1$

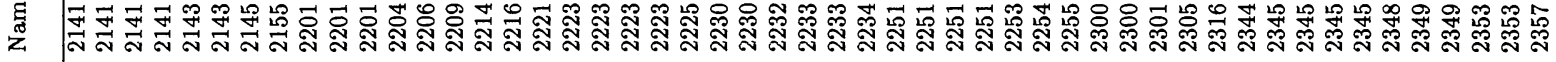

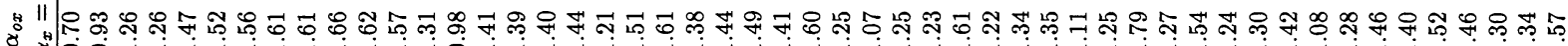

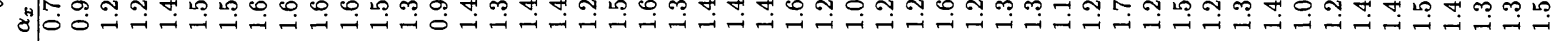

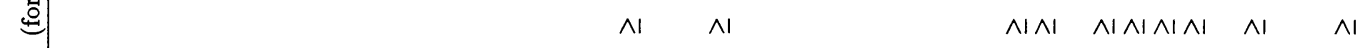

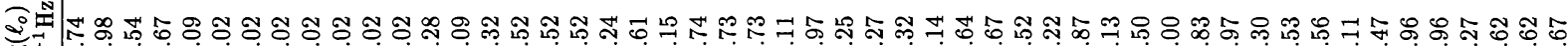

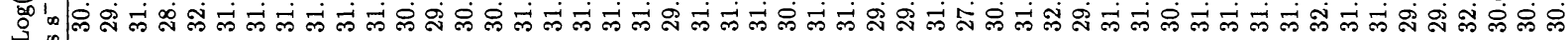

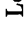

ֻ

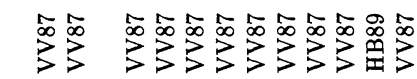

$\sum_{>}^{\infty} \sum^{\infty}$

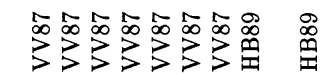

$\sum_{\infty}^{\infty}$

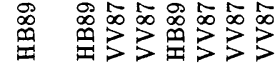

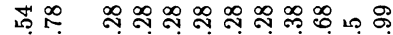

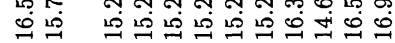

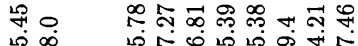

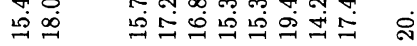

8 :

๓

7

๕ั

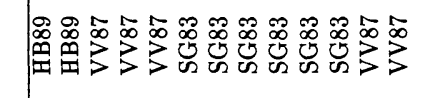

zั.

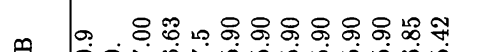

$\infty$

สิ่

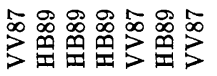

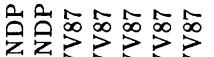

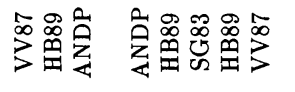

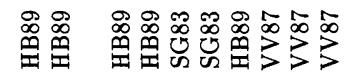

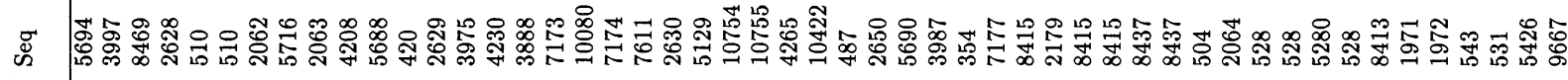

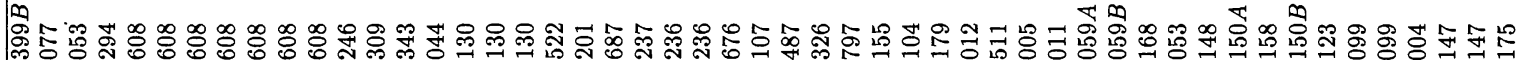

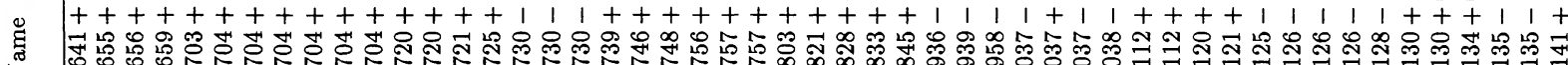

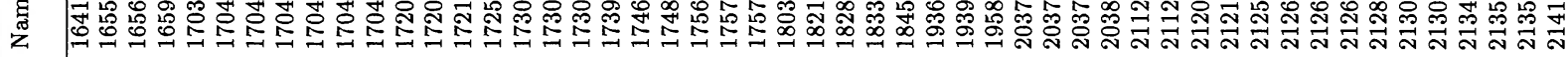


NoTES.- (1) Data used in subsequent analysis when multiple X-ray observations exist. (2) Magnitude quoted as $V$ in HB89 or VV87, but taken as $B$ in this paper on basis of original references. (3) Two observations of same quasar in two different X-ray fields lead to slightly different $N_{\mathrm{H}}$ 's and thereby slightly different optical extinctions and optical luminosities. (4) VV87 computer tapes or HB89 computer tapes give magnitude update relative to hard copy catalog. (5) $B$ magnitude from Hoag \& Smith 1977 as referenced in HB89. (6) $B$ magnitude from Burbidge \& Strittmatter 1972 as referenced in HB89. (7) $B$ magnitude from Kristian \& Sandage 1970 as referenced in VV87. (8) $B$ and $V$ magnitudes from Usher 1978 as referenced in HB89. (9) $B$ magnitude from Willis \& de Ruiter 1977 as referenced in VV87. (10) $B$ magnitude from Vaucher \& Weedman 1980 as referenced in HB89. (11) $B$ magnitude from Cohen et al. 1977 as referenced in VV87. (12) $B$ magnitude from Peterson, Bolton, \& Shimmins 1973 as referenced in HB89. (13) $B$ and $V$ magnitudes from Rafanelli $\&$ Schulz 1983 as referenced in VV87. (14) Original reference quotes magnitude as $R$; taken as $V$ here due to lack of additional data. (15) No published data (other than HB89) found to verify magnitude or band; HB89 value taken as $V$ magnitude. (16) $V$ magnitude from Hunstead, Murdoch, \& Shabbrook 1978 as referenced in HB89. (17) $B$ and $V$ magnitudes from Usher 1981 as referenced in HB89. (18) $B$ and $V$ magnitudes from Formiggini et al. 1980 as referenced in HB89. (19) $B$ magnitude from Marshall et al. 1984 as referenced in HB89. (20) $B$ magnitude from Braccesi et al. 1970 as referenced in HB89. (21) $B$ magnitude from Véron et al. 1976 as referenced in HB89.

REFERENCES.-ANDP, Anderson 1990; H92, Hazard 1992; HB89, Hewitt \& Burbidge 1989; SG83, Schmidt \& Green 1983; SIMBA, SIMBAD on-line catalogue; SW80, Sramek \& Weedman 1980; VV87, Véron-Cetty \& Véron 1987.

${ }^{a}$ This table is also published in computer-readable form in the AAS CD-ROM Series, Vol. 2.

where $\nu_{x}$ corresponds to $2 \mathrm{keV}$ and $\nu_{\text {opt }}$ corresponds to $2500 \AA$ in the quasar's rest frame, $l_{x}$ is the spectral luminosity at $2 \mathrm{keV}$ in units of $\mathrm{ergs}^{-1} \mathrm{~Hz}^{-1}$ assuming $\alpha_{x}=0.5$ (Tananbaum et al. 1979). For multiple X-ray observations of a given object, Table 6 contains multiple entry lines and appropriate values of $\alpha_{o x}$. Lower limits for $\alpha_{o x}$, corresponding to X-ray nondetections, are so indicated in Table 6.

\section{CHARACTERISTICS OF THE QUASAR SAMPLE}

As noted earlier, the quasars in the database are generally a heterogeneous sample but contain as subsets three complete samples: PG (optically selected), 3CR (radio-selected), and BF (optically selected). We describe the characteristics of the full set of 514 objects with the complete samples shown individually for comparison. Figure 2 shows the redshift distributions of the full, PG, 3CR, and BF samples respectively. It is clear that the database is dominated by low-redshift quasars with a long tail to higher redshift, the maximum redshift being 3.53. The X-ray and optical luminosity histograms (Figs. 3 and 4, respectively) show the range in spectral luminosity at $2 \mathrm{keV}$ and $2500 \AA$, respectively. The database covers more than six orders of magnitude in both quantities.

In Figure 5 the distribution of $\alpha_{o x}$ (effective X-ray-to-optical slope) values for the whole database and for each complete sample are displayed. X-ray nondetections, i.e., lower limits on $\alpha_{o x}$, are shaded. For our set of 514 "primary" observations, we have $328 \mathrm{X}$-ray detections and 186 nondetections. We further note that five of the nondetected objects were detected in one of our "nonprimary" observations. The PG sample is the most

TABLE 7

MEAN EQUIVALENT WIDTHS FOR THE PROMINENT EMISSION LINES

\begin{tabular}{ccc}
\hline \hline Line & $\begin{array}{c}\text { Wavelength } \\
(\AA)\end{array}$ & $\begin{array}{c}\text { Equivalent Width } \\
\left(W_{\lambda}, \AA\right)\end{array}$ \\
\hline $\mathrm{H} \beta \ldots \ldots \ldots$ & 4861 & 47 \\
$\mathrm{Mg}$ II $\ldots \ldots$. & 2798 & 27 \\
$\mathrm{C}$ III] $\ldots \ldots$. & 1909 & 17 \\
$\mathrm{C} \mathrm{IV} \ldots \ldots \ldots$ & 1549 & 32 \\
$\mathrm{Ly} \alpha \ldots \ldots$. & 1215 & 84 \\
\hline
\end{tabular}

representative of the quasar population as a whole; it has a mean $\alpha_{o x}$ of 1.5 and a range 1.0-2.0. We note, however, that this sample is biased toward bright optical luminosity as a result of its relatively bright apparent magnitude limit, which in turn affects its distribution of X-ray luminosities due to the dependence of $\alpha_{o x}$ on optical luminosity. Figure 6 shows the same histograms but with radio-loud objects shaded. In the optically selected samples (PG, BF), the figures show a tendency for the radio-loud objects to have smaller $\alpha_{o x}$ ( $L_{x}$ relatively greater) than the radio-quiet ones. This is a manifestation of the result that for a given optical luminosity, $\alpha_{o x}$ is lower if an object is radio loud ( $\mathrm{Ku}$, Helfand, \& Lucy 1980; Zamorani et al. 1981; Worrall et al. 1987).

\section{X-RAY AND OPTICAL LUMINOSITY RELATIONS}

The study by AT 86 of the dependence of the X-ray-to-optical luminosity ratio on optical luminosity and redshift is extended here using our significantly larger sample of optically selected quasars and Seyfert 1 galaxies.

\subsection{Object Selection}

The optically selected sample was assembled from the database by excluding certain sources: objects with flags " $\mathrm{X}$ " or "R" in the Hewitt \& Burbidge $(1987,1989)$ catalog of quasars, indicating X-ray or radio selection, were excluded unless there was also an "O" or " $\mathrm{C}$ " flag (indicating optical or UV-excess selection) or unless they were part of the PG or BF samples. Eight radio-loud objects flagged "C" by Hewitt \& Burbidge are excluded from our "optical" sample and are listed in Table 8. Six of these are included in the PHL Catalog of very blue objects (Haro \& Luyten 1962). For all eight objects, follow-up redshift studies and classification as quasars only resulted from independent discovery of strong radio emission. As noted in Table 1, 0112-017 (also UM 310) is included in the optically selected sample even though it is not flagged " $\mathrm{C}$ " or " $\mathrm{O}$ " by Hewitt \& Burbidge, because it was independently selected in a prism survey (Lewis, McAlpine, \& Weedman 1979).

This selection process ensures that radio-loud objects are included as optically selected only when they are independently selected in an optical survey. Our optically selected sample is comprised of 343 objects ( 179 detections and 164 upper limits) from the original 514. 

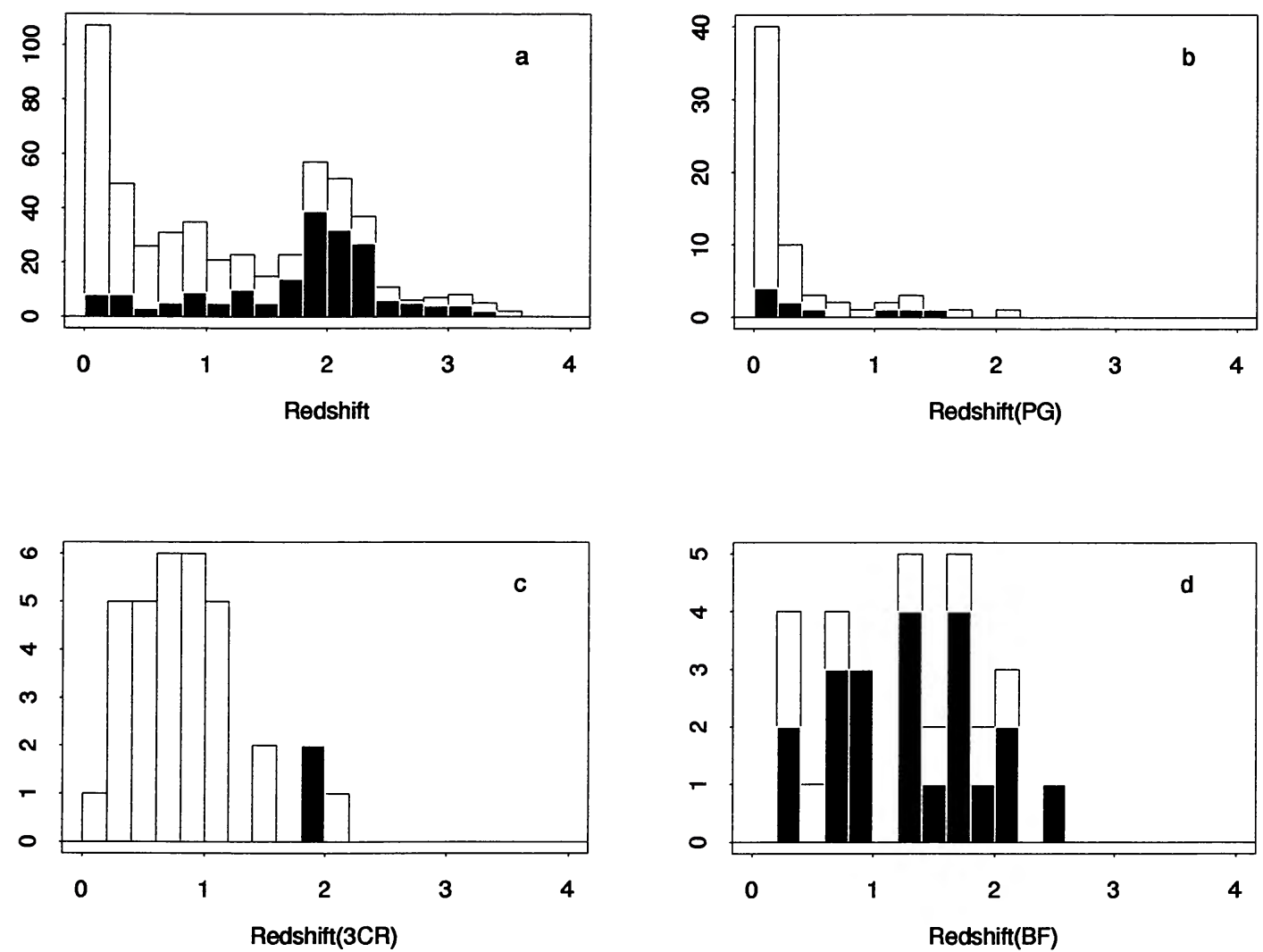

FIG. 2.-Histograms of the number of objects at each redshift for $(a)$ the full database and $(b)$ the PG, $(c)$ the $3 C R$, and $(d)$ the BF samples. X-ray upper limits are shown shaded.
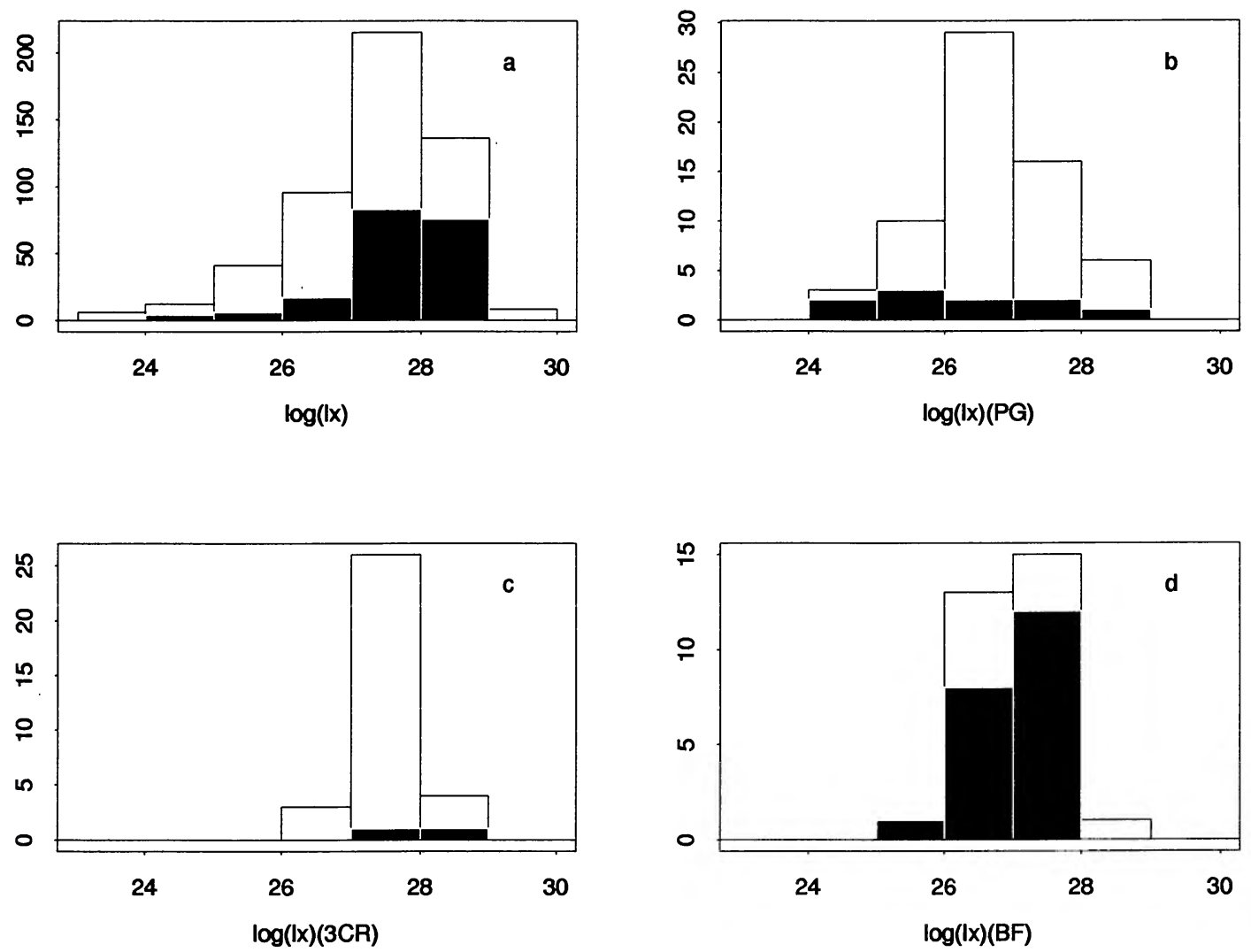

FIG. 3.-The range in $2 \mathrm{keV} \mathrm{X-ray} \mathrm{luminosity} \mathrm{present} \mathrm{in}(a)$ the full sample of 514 sources and $(b)$ the PG, $(c)$ the $3 \mathrm{CR}$, and $(d)$ the BF subsamples. $\mathrm{X}$-ray upper limits are shown shaded. The term $\log l_{x}$ is spectral luminosity at $2 \mathrm{keV}$ with $l_{x}$ in units of ergs s${ }^{-1} \mathrm{~Hz}^{-1}$ assuming $\alpha_{x}=0.5$. 

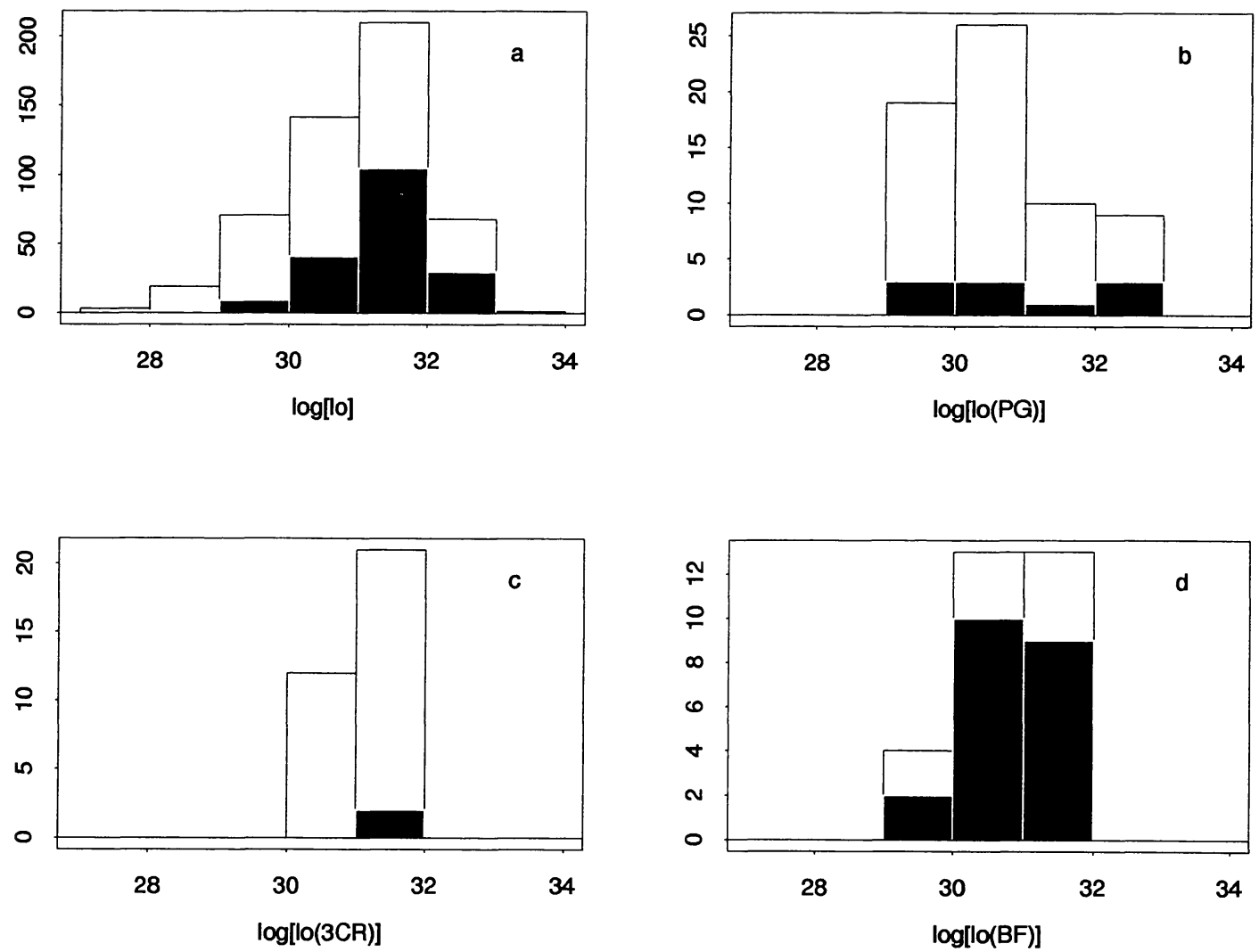

FIG. 4.-The range in $2500 \AA$ optical luminosity present in $(a)$ the full sample and $(b)$ the PG, $(c)$ the $3 C R$, and $(d)$ the BF subsamples. X-ray upper limits are shown shaded. The term $\log l_{o}$ is the spectral luminosity at $2500 \AA$ with $l_{o}$ in units of ergs s ${ }^{-1} \mathrm{~Hz}^{-1}$ derived from the optical magnitude under the assumption $\alpha_{o}=0.5$.
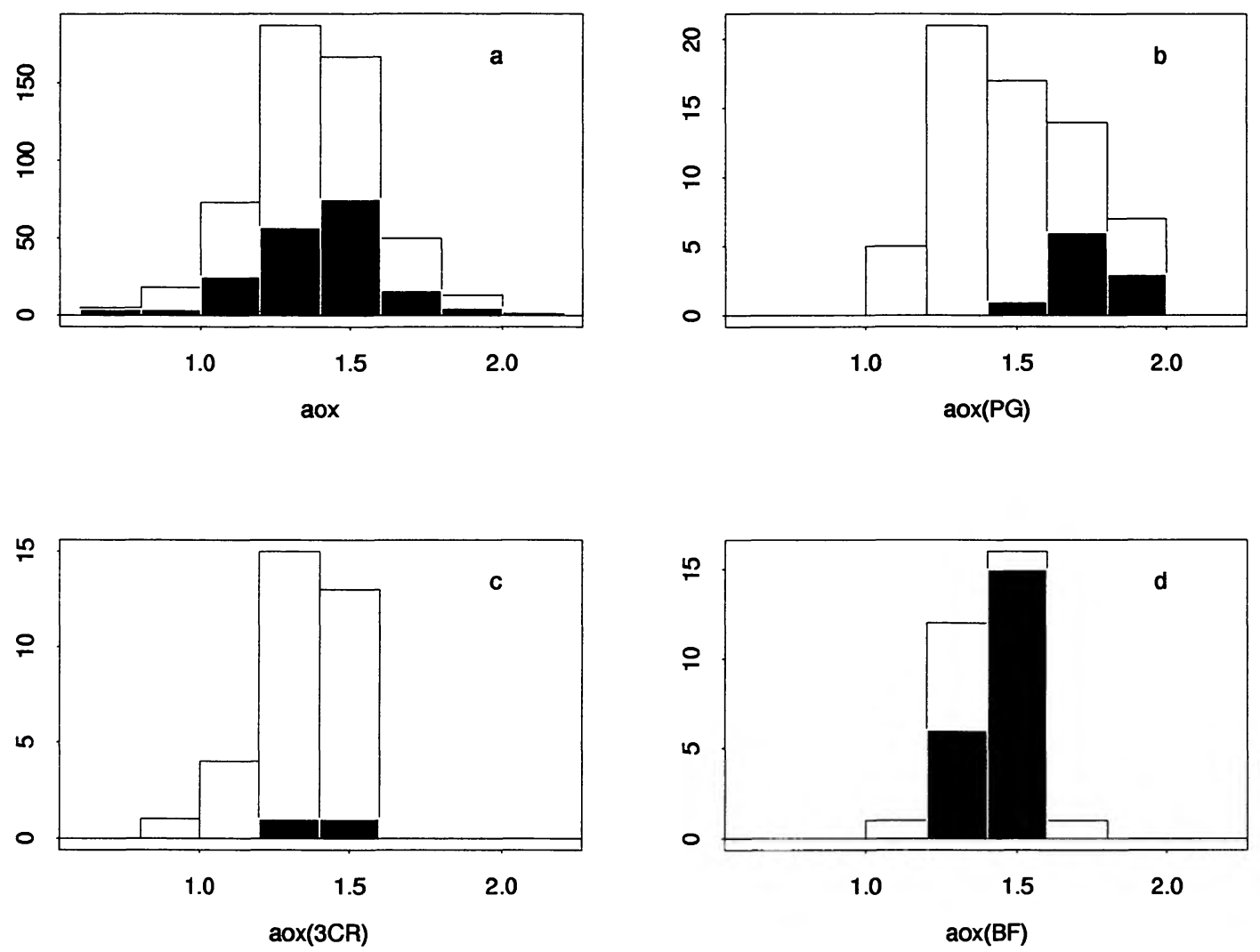

FIG. 5.-The range in effective optical to X-ray slope $\left(\alpha_{o x}\right)$ present in $(a)$ the full sample and $(b)$ the PG, $(c)$ the $3 \mathrm{CR}$, and $(d)$ the BF subsamples. Lower limits are shown shaded. 

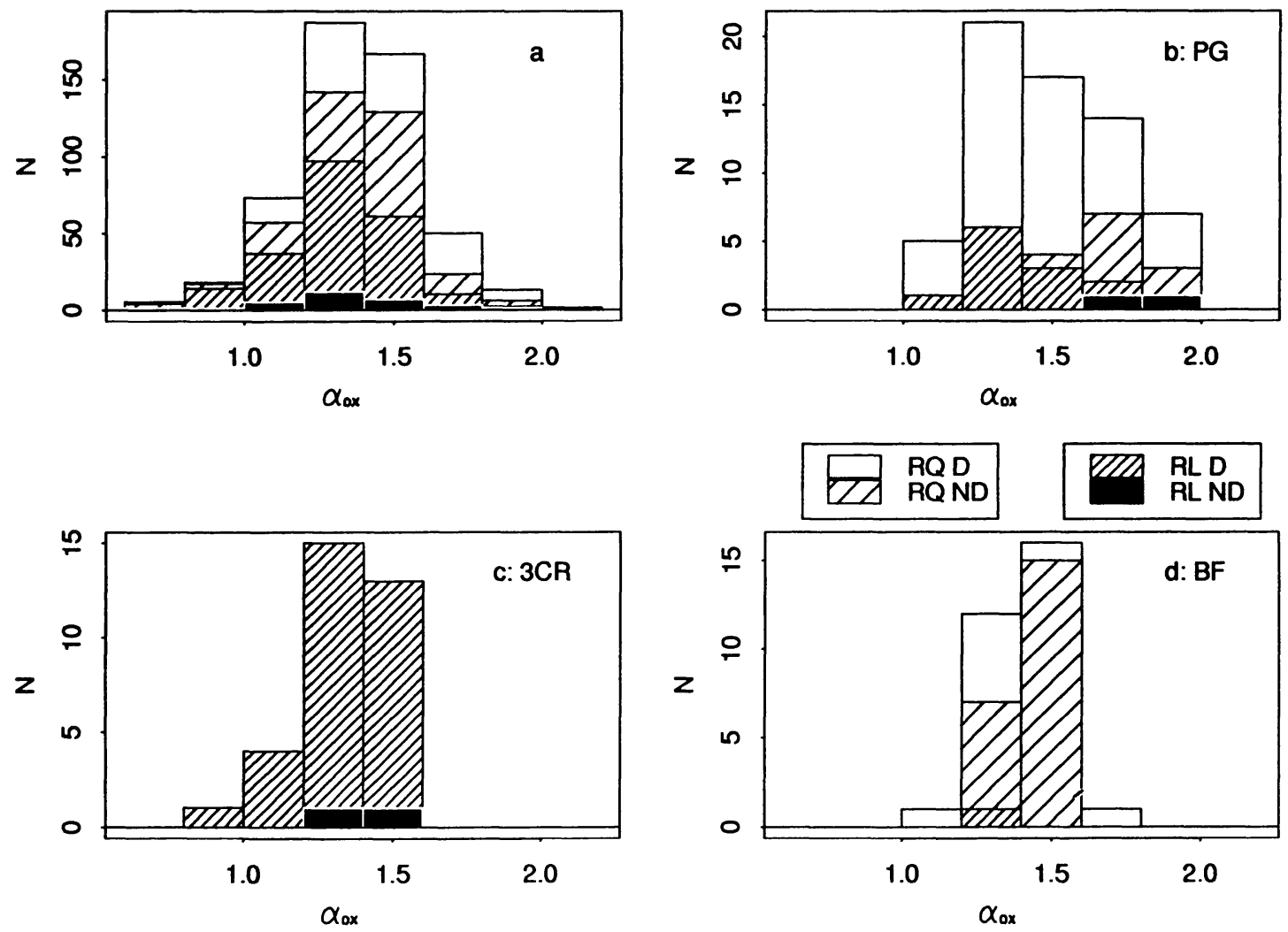

FIG. 6.-The range in effective optical to X-ray slope $\left(\alpha_{o x}\right)$ present in $(a)$ the full sample and $(b)$ the PG, $(c)$ the $3 \mathrm{CR}$, and $(d)$ the $\mathrm{BF}$ subsamples. RQ $=$ radio-quiet, $\mathrm{RL}=$ radio-loud, $\mathrm{D}=\mathrm{X}$-ray detections, $\mathrm{ND}=\mathrm{X}$-ray nondetections.

\subsection{Subsample Characteristics}

Figure 7 compares the distribution in redshift and $\log l_{o}$ for the current sample and that of AT86. One obvious difference between the two samples is our inclusion of lower luminosity objects. A K-S test for two-dimensional data (Press \& Teukolsky 1988) finds that the distribution of $z, \log l_{o}$ values differ between the current sample and that of AT86 $(0.2 \%$ of a chance occurrence).

\subsection{Dependence of X-ray Luminosity on Optical Luminosity}

We use the DB regression analysis method described in AT86 with the same functional form for the mean $\alpha_{o x}$,

$$
\begin{aligned}
\left\langle\alpha_{o x}\left(\log l_{o}, z\right)\right\rangle=A_{z}[\tau(z)- & 0.5] \\
& +A_{o}\left(\log l_{o}-30.5\right)+A,
\end{aligned}
$$

where $\tau(z)$ is the lookback time in units of the Hubble time (or $z /[1+z]$ for the $q_{0}=0$ cosmology assumed here).

For consistency with Avni \& Tananbaum (1982, hereafter AT82) and AT86, we assume that the $\alpha_{o x}$ residuals follow a Gaussian distribution about $\left\langle\alpha_{o x}\right\rangle$, and we compute the value of $\alpha_{o x}$ for each source using an X-ray spectral index of $\alpha_{x}=0.5$. Figures $8 a$ and $8 b$ show $90 \%$ confidence contours for two interesting parameters ( $\Delta S=4.6$ ) in the $A_{o}$ versus $A_{z}$ and $A_{o}$ versus
$A$ planes, respectively, for the new optically selected sample of 343 sources (solid line) and the earlier AT86 sample of 154 sources (dotted line). The revised contours are consistent with the earlier results but provide tighter constraints on the parameter values due to the larger sample. The decrease in size of the contours is not as great as would be expected (squareroot of ratio of number of sample objects in each linear dimension) if the larger sample were drawn from exactly the same population as the smaller. We attribute this to the differences between the ranges of $z$ and $\log l_{o}$ in the samples (see $\S 5.2$ ). Note that measurements of $l_{o}$ and $l_{x}$ revised with respect to AT86 have an insignificant effect on the fitted results. A total of 143 sources

TABLE 8

ObJects FlaGged as COLOR-SELECTED By HewitT \& BURBIDGe, BUT EXCLUDED FROM OUR OPTICALly SELECTED SAMPLE

\begin{tabular}{cl}
\hline \multicolumn{1}{c}{ Name } & \multicolumn{1}{c}{ Other Names } \\
\hline $0017+154 \ldots \ldots$ & PHL 2871, 3C R9 \\
$0056-001 \ldots \ldots$ & PHL 923, PKS \\
$0226-038 \ldots \ldots$ & PHL 1305, PKS \\
$0730+659 \ldots \ldots$ & W1 0730+659 \\
$1223+252 \ldots \ldots$ & TON 616, 4C 25.40 \\
$2128-123 \ldots \ldots$ & PHL 1598, PKS \\
$2134+004 \ldots \ldots$ & PHL 61, PKS \\
$2135-147 \ldots \ldots$ & PHL 1657, PKS \\
\hline
\end{tabular}




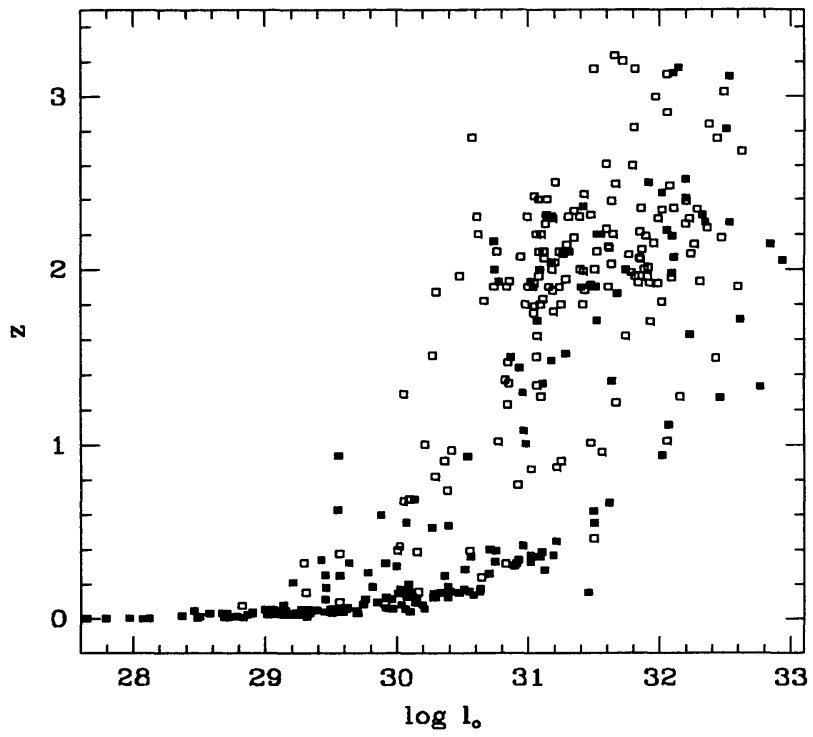

$$
\begin{array}{r}
\left\langle\alpha_{o x}\right\rangle=(0.11 \pm 0.02)\left(\log l_{o}-30.5\right)+(1.53 \pm 0.02) \\
\left\langle\log \left(\frac{l_{x}}{10^{26.5}}\right)\right\rangle=(0.71 \pm 0.05) \log \left(\frac{l_{o}}{10^{30.5}}\right) \\
+(0.018 \pm 0.065) .
\end{array}
$$

Figure 9 shows the values of $\log l_{x}$ and $\log l_{o}$ for our sample objects with the fit of equation (13) superposed. The scatter of the data about the best-fit line suggests visually that something more complicated than a linear dependence of $l_{x}$ on $l_{o}$ may give a better fit; this is investigated in a forthcoming paper (Avni, Worrall, \& Morgan 1994). Figure 10 illustrates that the X-ray and optical flux densities are correlated, confirming that a correlation between $\log l_{x}$ and $\log l_{o}$ is not an artifact of common distance spreading along each axis.

AT86 found that although a Gaussian form to the residuals was acceptable, a skew distribution with a longer tail at high $\alpha_{o x}\left(\right.$ low $l_{x}$ ) and a shorter tail at low $\alpha_{o x}$ (high $l_{x}$ ) gave a better fit. This change was found to reduce the normalization but not the shape of the dependence on $l_{o}$. DB regression analysis can also be applied using a nonparametric form for the residuals (Avni et al. 1980). This method gives a similar dependence of $\alpha_{o x}$ on $\log l_{o}$, and, by fitting the mean $\alpha_{o x}$ for different bands of $\log l_{o}$, the shape of the dependence of $\alpha_{o x}$ on $\log l_{o}$ can be investigated. The nonparametric approach has been investigated further and applied to the present sample by Avni, Worrall, \& Morgan (1991, 1994) who show how the resulting uncertainties (although not the best fits) depend on the allowed range for the underlying distribution of residuals and who point out that errors may be underestimated if care is not taken in applying such methods (see also Anderson 1985).

Margon et al. (1992) have found a very similar dependence of $\alpha_{o x}$ on $\log l_{o}$ to our fit of equation (12). Their slope is $0.11 \pm$ 0.01 (error presumed to be $1 \sigma$ for one interesting parameter) from fitting measurements of 146 objects which were observed with the IPC and taken from a complete quasar sample. While some of their objects are in common with our analysis, it is significant that the analysis method they employ, "image stacking," is quite different from ours, and yet the results agree very well. Marshall (1992) also obtains a similar result to that given here from a further analysis of the quasar sample of AT86. Marshall's treatment includes the uncertainties in the detected fluxes as well as the probability distribution associated with the nondetections.

Low-luminosity objects show increased dispersion in their dependence of $l_{x}$ on $l_{o}$, probably due to a combination of varying degrees of starlight contamination which would affect $l_{o}$ and intrinsic absorption (believed to be largest in low-luminosity sources) having been ignored in the calculations of $l_{x}$ (Worrall 1987). If we restrict our sample to exclude the lowest luminosity AGNs $\left(\log l_{o}<29.0\right)$, we find that the results change marginally in the direction of a larger $A_{o}$ (i.e., a flatter dependence on $l_{o}$; see Figs. $11 a$ and $\left.11 b\right)$. The new best-fit parameters are $A_{o}=0.15 \pm 0.04, A_{z}=-0.08 \pm 0.17, A=$ $1.49 \pm 0.04$, with $\sigma=0.23 \pm 0.02$. Still no dependence on redshift is required by the fit. This change is consistent with 

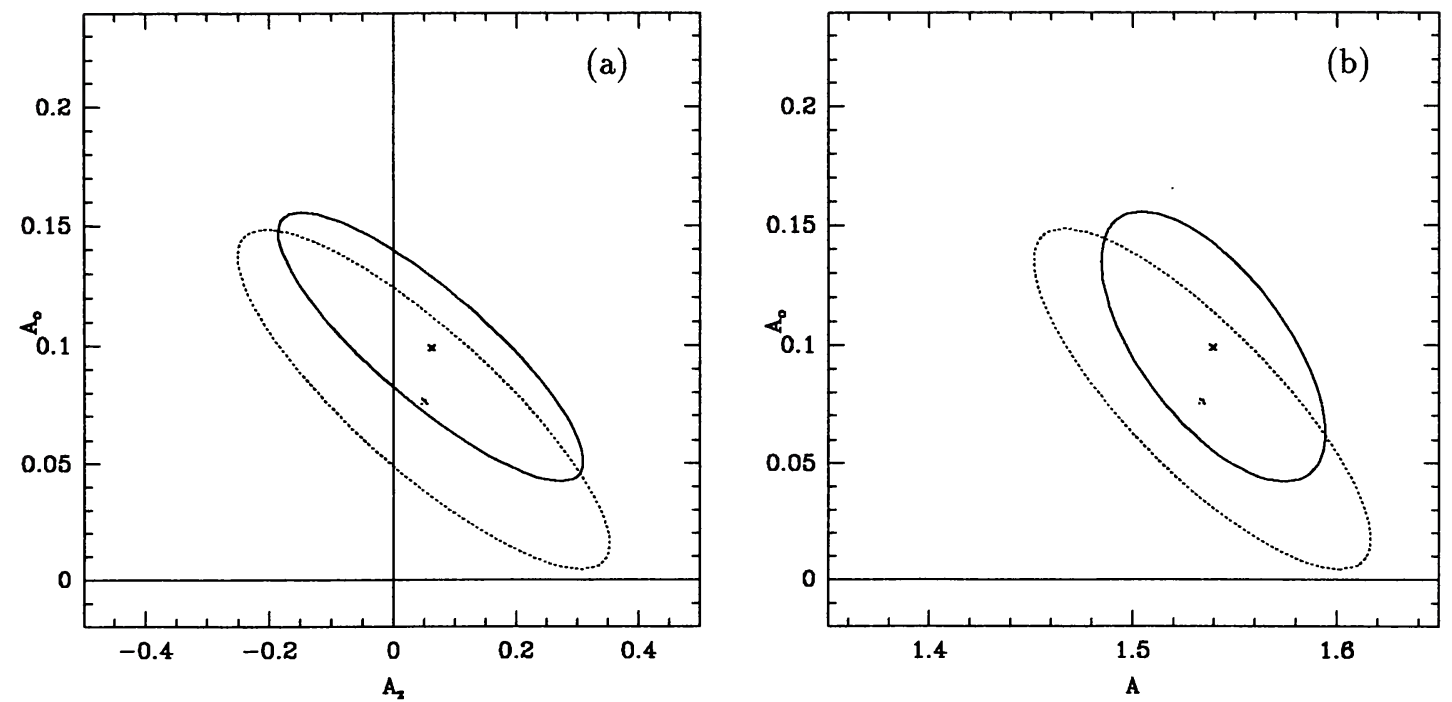

FIG. 8.-The $90 \%$ confidence ( $\Delta S=4.6$ ) contours for $(a) A_{o}, A_{z}$, (with $A$ free), and ( $\left.b\right) A_{o}, A$, (with $A_{z}$ free), from fitting the dependence of $\alpha_{o x}$ on $z$ and $\log l_{o}$ (eq. [11]). Contours using the previously analyzed smaller dataset of AT86 are shown (dotted lines) for comparison.

contamination by starlight at low $l_{o}$ steepening the dependence of $l_{o}$. This is clear in Figure 9 where the low luminosity objects have systematically high $l_{o}$.

\subsection{Dependence on X-Ray Spectral Index}

Although we assumed a value of $X$-ray spectral energy index $\alpha_{x}=0.5$ in our analysis, for comparison with earlier work, it is

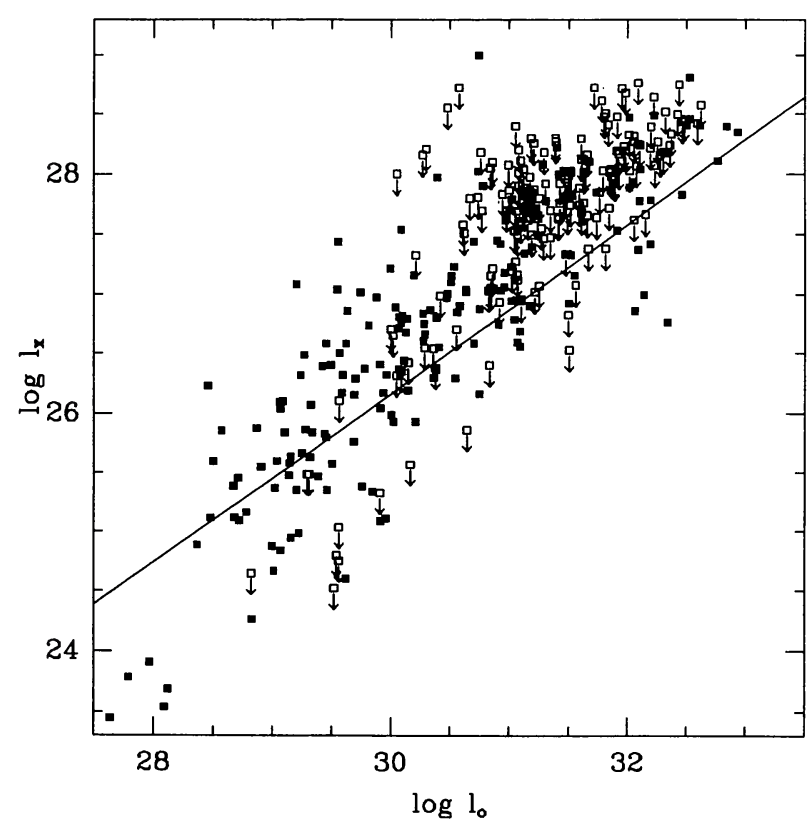

FIG. 9.-The relation between X-ray and optical luminosity for the new optically selected sample of 343 sources, with best-fit model assuming no redshift dependence. Luminosities are in units of ergs $\mathrm{s}^{-1} \mathrm{~Hz}^{-1}$ at $2 \mathrm{keV}$ and $2500 \AA$ for $l_{x}$ and $l_{o}$, respectively. Detections are indicated by filled squares and $\mathrm{X}$-ray upper limits by open squares with arrows attached. now known that, for the brightest X-ray AGNs measured with the IPC, a slope of $0.5-0.7$ is more typical for radio-loud objects and $\sim 1.0$ for their radio-quiet counterparts (Wilkes \& Elvis 1987; Canizares \& White 1989; Worrall 1989; Brunner et al. 1989). Figure 12 shows that our conclusion that $\alpha_{o x}$ depends primarily on $\log l_{o}$ rather than redshift is relatively insensitive to our choice of $\alpha_{x}$. A change in $\alpha_{x}$ affects $A_{z}$ and $A$ but not $A_{o}$. This is because $\alpha_{o x}$ jointly depends on $\alpha_{x}$ and $z$ through

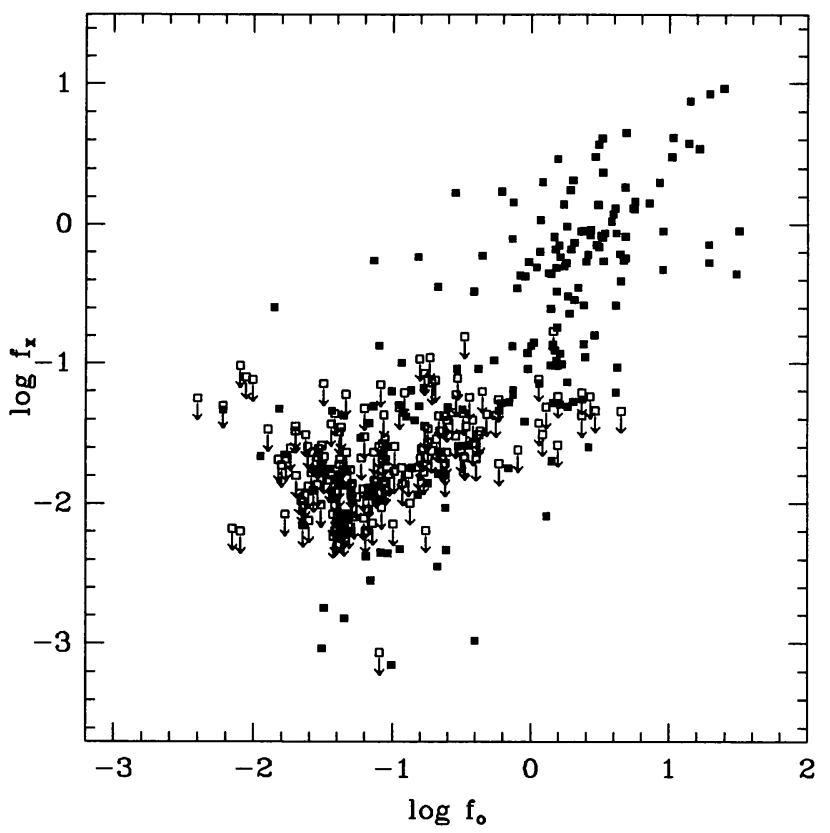

FIG. 10.-A dependence of X-ray flux density $(\mu \mathrm{Jy})$ on optical flux density ( $\mathrm{mJy}$ ) shows that the $\log l_{x}, \log l_{o}$ correlation of Fig. 9 is not induced merely by common distance spreading along each axis. Detections are indicated by filled squares and $\mathrm{X}$-ray upper limits by open squares with arrows attached. 

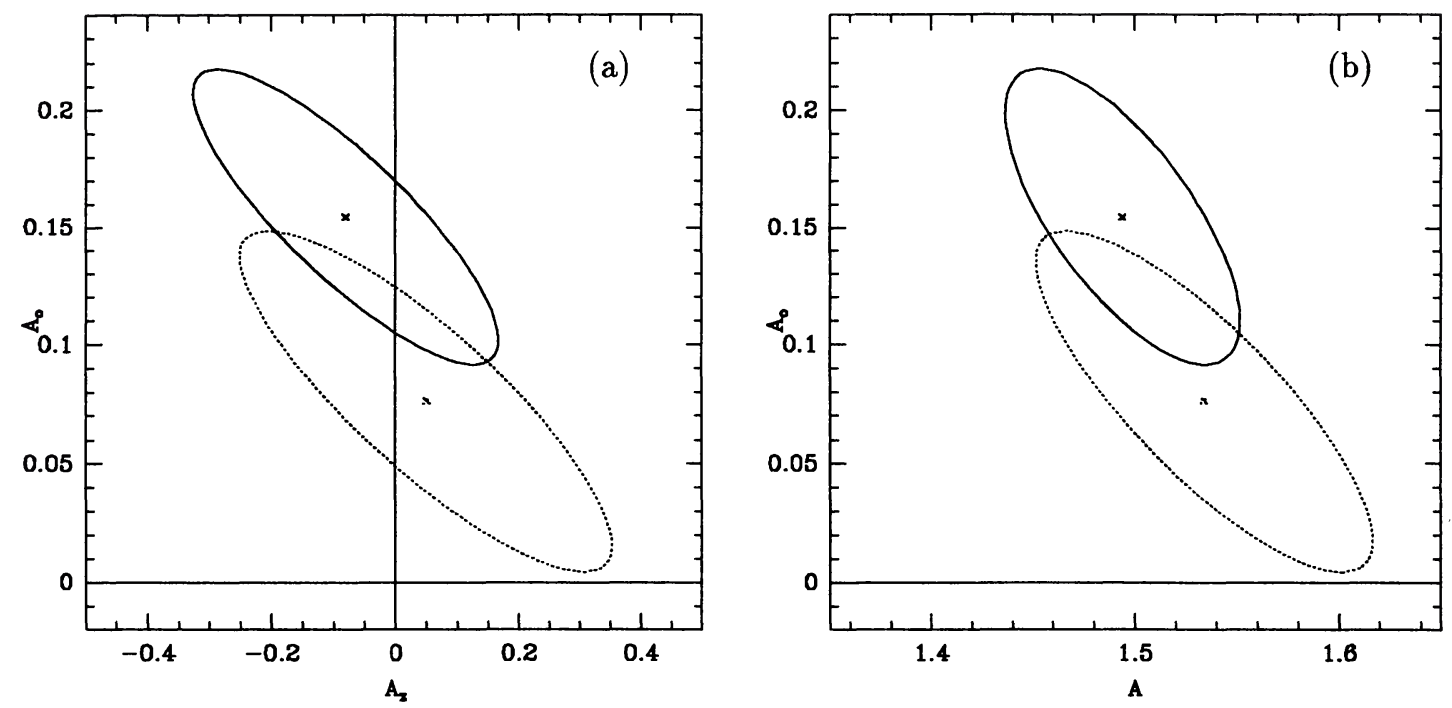

FIG. 11.-The 90\% confidence ( $\Delta S=4.6$ ) contours for $(a) A_{o}, A_{z}$, and $(b) A_{o}, A$, from fitting the dependence of $\alpha_{o x}$ on $z$ and $\log l_{o}$, where low-luminosity AGNs $\left(\log l_{o}<29.0\right)$ have been excluded. As in Fig. 8, contours using the previously analyzed smaller dataset of AT86 are shown $($ dotted lines) for comparison.

the $K$-correction term of the $\mathrm{X}$-ray luminosity calculation ( see the form of eq. [6]), whereas there is no joint dependence on $\alpha_{x}$ and $l_{o}$. (However, note that in fits to eq. [11] with $A_{z}$ explicitly set to zero, changes in $\alpha_{x}$ affect both $A_{o}$ and $A$; this is because if there is no redshift term in the fitting function to accommodate the joint dependence of $\alpha_{o x}$ on $\alpha_{x}$ and $z$, the optical luminosity term becomes indirectly affected due to a dependence of $z$ on $l_{o}$ [Fig. 7].)

\subsection{Difference between Radio-loud and Radio-quiet Sources}

Figure 13 shows separately the redshift versus $\log l_{o}$ figures for the 291 radio-quiet and 52 radio-loud sources of the opti- cally selected sample. A K-S test finds that these subsamples differ in their distributions of $z, \log l_{o}(0.5 \%$ of a chance occurrence), although the incompleteness of our samples precludes any cosmological significance from being attributed to this difference.

We exclude low-luminosity objects and compare samples for $\log l_{o}>29.0$ in order to be somewhat comparable with Worrall et al. (1987) who actually used an even higher threshold of $\log l_{o}>29.95$. The radio-loud and radio-quiet subsets are consistent with $A_{z}=0$ and the same dependence on $\log l_{o}$ (i.e., same $A_{o}$ ), but fit very different normalization constants, $A$ (Figs. $14 a$ and $14 b$ ). This confirms earlier results that radioloud sources produce more $\mathrm{X}$-rays relative to their optical lumi-
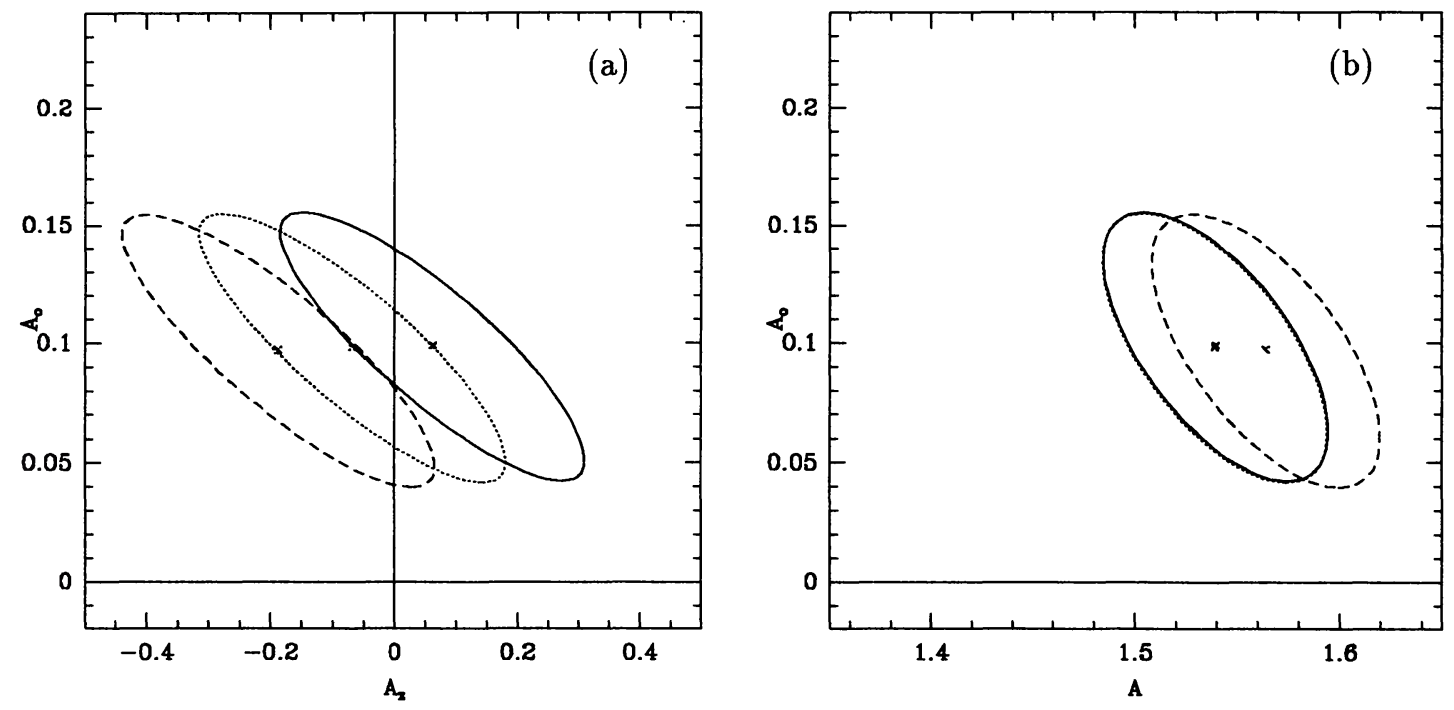

Fig. 12.-The 90\% confidence ( $\Delta S=4.6$ ) contours for $(a) A_{o}, A_{z}$, and $(b) A_{o}, A$, from fitting the dependence of $\alpha_{o x}$ on $z$ and log $l_{o}$, for three different assumptions about the X-ray spectral index: $\alpha_{x}=0.5$ (solid line; same as Fig. 10), $\alpha_{x}=1.0$ (dotted line), and $\alpha_{x}=1.5$ (dashed line). 

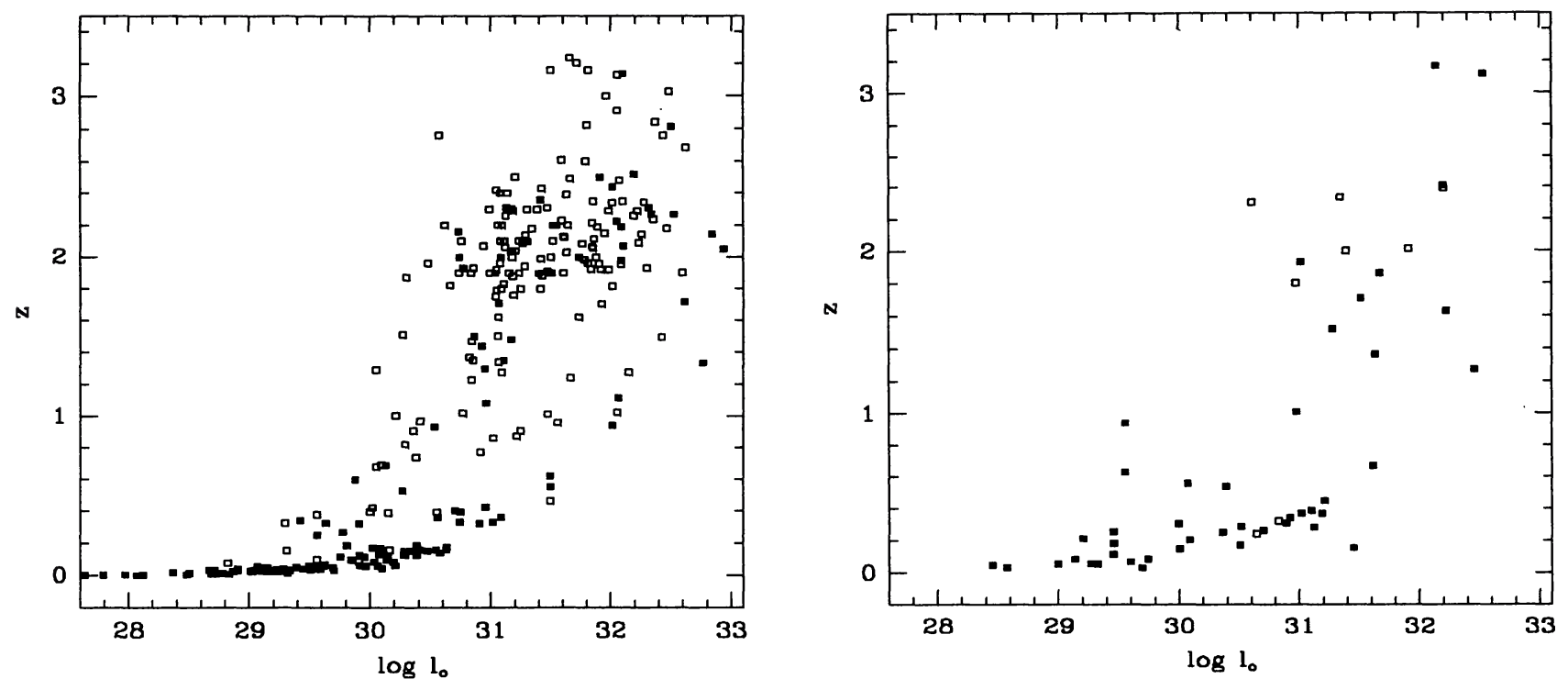

FIG. 13.-The distribution in redshift and $\log l_{o}$ of objects in the new optically selected sample, divided into radio-quiet (upper) and radio-loud (lower) subsamples. Following Zamorani et al. (1981), a source is radio loud if its spectral index between $5 \mathrm{GHz}$ and $2500 \AA$ in the source frame is larger than 0.35 . The term $\log l_{o}$ is the logarithm of the spectral luminosity at $2500 \AA$, in units of ergs $\mathrm{s}^{-1} \mathrm{~Hz}^{-1}$. X-ray detections are shown as filled symbols; open symbols are $\mathrm{X}$-ray upper limits.
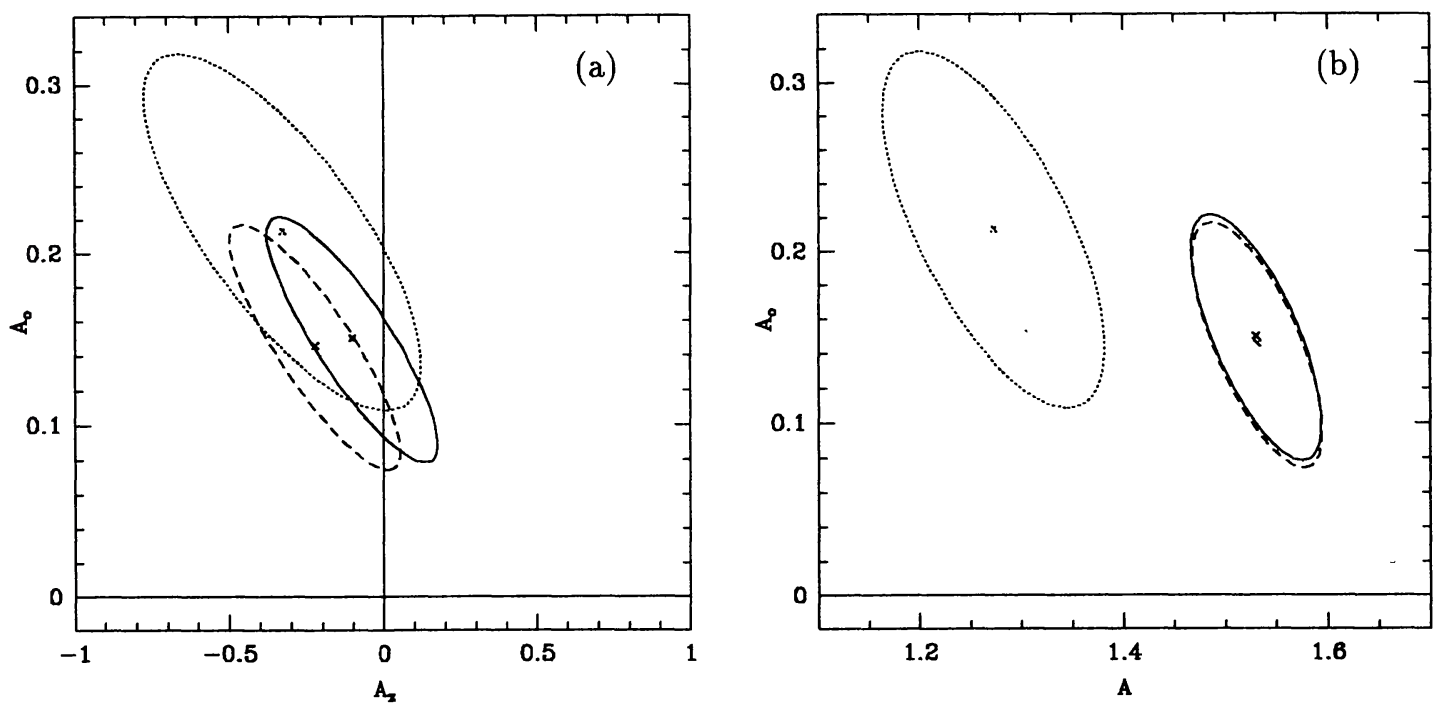

Fig. 14. - The $90 \%$ confidence contours $(\Delta S=4.6)$ for the 272 radio-quiet $\left(\alpha_{x}=0.5\right.$, solid line; $\alpha_{x}=1.0$, dashed line $)$ and 49 radio-loud $\left(\alpha_{x}=0.5\right.$, dotted line) quasars with $\log l_{o}>29.0$. The low-luminosity sources are excluded because they bias the fit and are predominantly only radio quiet (Fig. 13). $(a)$. $A_{o}$ vs. $A_{z}$. The radio-quiet and radio-loud subsamples are consistent with the same $A_{o}$ and $A_{z}=0 .(b) . A_{o}$ vs. $A$. The radio-quiet and radio-loud subsamples are consistent with the same $A_{o}$ (dependence on $\log l_{o}$ ), but fit different normalization constants, confirming earlier results that a source which is radio loud will produce more X-rays relative to its optical luminosity. 
nosity (i.e., radio-loud sources have a lower value of $A$, and thus a smaller [ flatter] $\alpha_{o x}$; Ku et al. 1980; Zamorani et al. 1981; Worrall et al. 1987). We see from Figure $14 b$ that allowing for a different spectral slope for the two populations $(0.5$ for radio-loud and 1.0 for radio-quiet; see $\S 5.4$ ) does not account for the difference in normalization.

We would like to thank Richard Kondo for his work on coding the automated analysis procedures and Paul Martenis and Susannah Hopkins for assistance in its execution. We also thank Rick Harnden, Frank Primini, and John McSweeney for assistance with computation of the appropriate background levels for deep survey exposures; Mark Birkinshaw and Martin Elvis for a careful reading of the manuscript; and Karen Modestino for her professional expertise in preparation of the text and tables. This work was supported by NASA contracts NAS8-30751 and NAS8-39073.

Tables 1, 2, 3, 5, and 6 also are published in computer-readable form in the AAS CD-ROM Series, Vol. 2.

\section{REFERENCES}

Allen, C. W. 1973, Astrophysical Quantities (London: Athlone), 264 Anderson, S. F. 1985, Ph.D. thesis, Univ. of Washington

. 1990, private communication

Avni, Y., Soltan, A., Tananbaum, H., \& Zamorani, G. 1980, ApJ, 238, 800

Avni, Y., \& Tananbaum, H. 1982, ApJ, 262, L17 (AT82) . 1986, ApJ, 305, 83 (AT86)

Avni, Y., Worrall, D. M., \& Morgan, W. A., Jr. 1991, Inst. Math. Stat. Bull., 20, 260 1994, in preparation

Braccesi, A., Formiggini, L., \& Gandolfi, E. 1970, A\&A, 5, 264

Braccesi, A., Lynds, R., \& Sandage, A. 1968, ApJ, 152, L105

Bregman, J. N., Glassgold, A. E., Huggins, P. J., \& Kinney, A. L. 1985, ApJ, 291, 505

Brunner, H., Worrall, D. M., Wilkes, B. J., \& Elvis, M. 1989, in Proc. 23rd

ESLAB Symposium (ESA SP-296) (Noordwijk: ESA) 905

Burbidge, E. M., \& Strittmatter, P. A. 1972, ApJ, 174, L57

Burstein, D., \& Heiles, C. 1978, ApJ, 225, 40

Canizares, C. R., \& White, J. L. 1989, ApJ, 339, 27

Cohen, A. M., Porcas, R. W., Browne, I. W. A., Daintree, E. J., \& Walsh, D. 1977 , MNRAS, 84,1

Colla, G., et al. 1970, A\&AS, 1, 281

Formiggini, L., Zitelli, V., Bonoli, F., \& Braccesi, A. 1980, A\&AS, 39, 129

Giacconi, R., et al. 1979, ApJ, 230, 540

Green, R. F., Schmidt, M., \& Liebert, J. 1986, ApJS, 61, 305

Harnden, F. R., Fabricant, D. G., Harris, D. E., \& Schwarz, J. 1984, Smithsonian Astrophys. Obs. Spec. Rep., 393

Haro, G., \& Luyten, W. J. 1962, Bol. Obs. Tonantzintla y Tacubaya, 13(22), 37

Harris, D. E., et al. 1990, The Einstein Observatory Catalog of IPC X-Ray Sources (NASA TM-108401)

Hayes, D. S., \& Latham, D. W. 1975, ApJ, 197, 593

Hazard, C. 1992, private communication

Heiles, C., \& Cleary, M. N. 1979, Australian J. Phys. Astrophys. Suppl., No. 47,1

Hewitt, A., \& Burbidge, G. 1987, ApJS, 63, 1 (HB87) . 1989, ApJS, 69, 1 (HB89)

Hoag, A. A., \& Smith, M. G. 1977, ApJ, 217, 362

Hunstead, R. W., Murdoch, H. S., \& Shabbrook, B. B. 1978, MNRAS, 185,149

Kristian, J., \& Sandage, A. 1970, ApJ, 162, 391
Ku, W. H-M., Helfand, D. J., \& Lucy, L. B. 1980, Nature, 288, 323

Lewis, D. W., McAlpine, G. M., \& Weedman, D. W. 1979, ApJ, 233, 787

Margon, B., Anderson, S. F., Wu, X., Green, P. J., \& Foltz, C. B. 1992, in

X-Ray Emission from AGN and the Cosmic X-ray Background, ed. W.

Brinkman \& J. Trümper (Garching: Max-Planck-Institut), 81

Marshall, H. L. 1983, Ph.D. thesis, Harvard Univ.

. 1992, in Statistical Challenges in Modern Astronomy, ed. E. D. Feigelson \& G. J. Babu (Berlin: Springer-Verlag), 247

Marshall, H. L., Avni, Y., Braccesi, A., Huchra, J. P., Tananbaum, H., Zamorani, G., \& Zitelli, V. 1984, ApJ, 283, 50

Oke, J. B. 1974, ApJS, 27, 21

Peterson, B. A., Bolton, J. G., \& Shimmins, A. T. 1973, Astrophys. Lett., 15,109

Press, W. H., \& Teukolsky, S. R. 1988, Comput. Phys., July/August, 74

Rafanelli, P., \& Schulz, H. 1983, A\&A, 117, 109

Schmidt, M. 1968, ApJ, 151, 393

Schmidt, M., \& Green, R. F. 1983, ApJ, 269, 352

Sramek, R. A., \& Weedman, D. W. 1980, ApJ, 238, 435

Stark, A. A., Gammie, C. F., Wilson, R. W., Bally, J., Linke, R. A., Heiles, C., \& Hurwitz, M. 1992, ApJS, 79, 77

Tananbaum, H., et al. 1979, ApJ, 234, L9

Tananbaum, H., Avni, Y., Green, R. F., Schmidt, M., \& Zamorani, G. 1986, ApJ, 305, 57

Tananbaum, H., Wardle, J. F. C., Zamorani, G., \& Avni, Y. 1983, ApJ, 268,60

Usher, P. D. 1978, ApJ, 222, 40 1981, ApJS, 46, 117

Vaucher, B. G., \& Weedman, D. W. 1980, ApJ, 240, 10

Véron, M. P., Véron, P., Algie, R. L., \& Gent, H. 1976, A\&A, 47, 401

Véron-Cetty, M. P., \& Véron, P. 1987, A Catalogue of Quasars and Active

Nuclei (ESO Sci. Rep. 5) (3d ed.; Garching: ESO) (VV87)

Wilkes, B. J. 1986, MNRAS, 218, 331

Wilkes, B. J., \& Elvis, M. 1987, ApJ, 323, 243

Willis, A. G., \& deRuiter, H. R. 1977, A\&AS, 29, 103

Worrall, D. M. 1987, ApJ, 318, 188

. 1989, in Proc. 23rd ESLAB Symposium (ESA SP-296) (Noordwijk: ESA), 719

Worrall, D. M., Giommi, P., Tananbaum, H., \& Zamorani, G. 1987, ApJ, 313, 596

Zamorani, G., et al. 1981, ApJ, 245, 357 